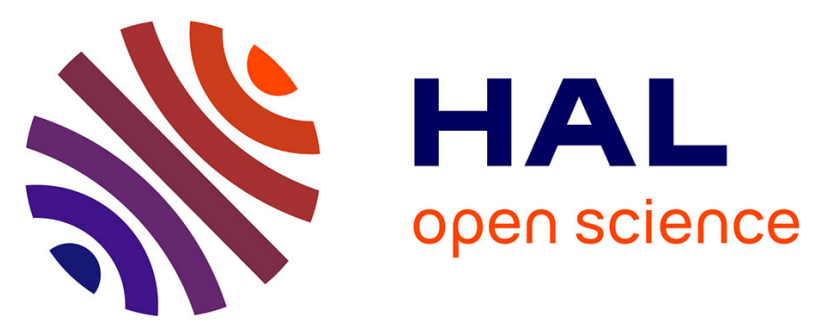

\title{
Latest experimental and theoretical advances in the production of negative ions in caesium-free plasmas
}

F. Taccogna, S. Béchu, A. Aanesland, P. Agostinetti, R. Agnello, S. Aleiferis, T. Angot, V. Antoni, M. Bacal, M. Barbisan, et al.

\section{- To cite this version:}

F. Taccogna, S. Béchu, A. Aanesland, P. Agostinetti, R. Agnello, et al.. Latest experimental and theoretical advances in the production of negative ions in caesium-free plasmas. Eur.Phys.J.D, 2021, 75 (8), pp.227. 10.1140/epjd/s10053-021-00228-y . hal-03325804

\section{HAL Id: hal-03325804 \\ https://hal.science/hal-03325804}

Submitted on 18 Nov 2021

HAL is a multi-disciplinary open access archive for the deposit and dissemination of scientific research documents, whether they are published or not. The documents may come from teaching and research institutions in France or abroad, or from public or private research centers.
L'archive ouverte pluridisciplinaire HAL, est destinée au dépôt et à la diffusion de documents scientifiques de niveau recherche, publiés ou non, émanant des établissements d'enseignement et de recherche français ou étrangers, des laboratoires publics ou privés. 


\title{
Latest experimental and theoretical advances in the production of negative ions in caesium-free plasmas
}

F. Taccogna ${ }^{1,2}$, S. Bechu ${ }^{3}$, A. Aanesland ${ }^{4}$, P. Agostinetti ${ }^{5}$, R. Agnello ${ }^{6}$, S. Aleiferis ${ }^{3,7,8}$, T. Angot ${ }^{9}$, V. Antoni ${ }^{5}$, M. Bacal ${ }^{10}$, M. Barbisan ${ }^{5}$, J. Bentounes ${ }^{3,11}$, A. Bès ${ }^{3}$, M. Capitelli ${ }^{1}$, G. Cartry ${ }^{9}$, M. Cavenago ${ }^{12}$, R. Celiberto $^{13,1}$, G. Chitarin ${ }^{5}$, R. Delogu ${ }^{5}$, A. De Lorenzi ${ }^{5}$, F. Esposito ${ }^{1}$, M. Fadone ${ }^{5}$, N. Ferron ${ }^{5}$, G. Fubiani ${ }^{14}$, I. Furno $^{6}$, L. Gavilan ${ }^{15}$, P. Guittienne ${ }^{6}$, A. Howling ${ }^{6}$, R. Jacquier ${ }^{6}$, A. Laricchiuta ${ }^{1}$, J. M. Layet ${ }^{9}$, J. L. Lemaire ${ }^{16}$, S. Longo ${ }^{1}$, B. Maurice ${ }^{9}$, P. Minelli1 ${ }^{1,2}$, M. Minissale ${ }^{9}$, M. Mitrou $^{7,3}$, R. Moussaoui ${ }^{9}$, A. Pimazzoni ${ }^{12}$, C. Poggi ${ }^{5}$, D. Rafalskyi ${ }^{4}$, E. Salomon 9 , E. Sartori ${ }^{5}$, M. Sasao ${ }^{17}$, G. Serianni ${ }^{5}$, E. Spada ${ }^{5}$, S. Suweis ${ }^{18}$, P. Svarnas ${ }^{7}$, L.

Tahri $^{9}$, M. Ugoletti ${ }^{5}$, V. Variale ${ }^{2}$, P. Veltri ${ }^{19}$

${ }^{1}$ Institute for Plasma Science and Technology, CNR, via Amendola 122/D, 70126 Bari, Italy

${ }^{2}$ INFN-BA, via Amendola 173, 70126 Bari, Italy

${ }^{3}$ Laboratoire de Physique Subatomique et de Cosmologie, Université Grenoble-Alpes, CNRS/IN2P3, F-38026

Grenoble, France

${ }^{4}$ ThrustMe, 4bis Rue des Petits Ruisseaux, 91370 Verrieres-le-Buisson, France

${ }^{5}$ Consorzio RFX (CNR, ENEA, INFN, Università di Padova, Acciaierie Venete Spa), Padova, Italy

${ }^{6}$ Ecole Polytechnique Fédérale de Lausanne (EPFL), Swiss Plasma Center (SPC), CH-1015 Lausanne, Switzerland

${ }^{7}$ High Voltage Laboratory, Electrical and Computer Engineering Department, University of Patras, 26504 Rion-Patras, Greece

${ }^{8}$ EUROfusion consortium, JET, CCFE, Culham, Science Centre, Abingdon, Ox14 3DB, UK

${ }^{9}$ Aix-Marseille Université, CNRS, PIIM, UMR 7345, Centre Scientifique de Saint Jéome, Case 241, 13397 Marseille

Cedex 20, France

${ }^{10}$ UPMC, Ecole Polytechnique, LPP, UMR CNRS 7648, Palaiseau, France

${ }^{11}$ Abdelhamid Univ. IBN Badis Mostaganem, Algeria

12 INFN-LNL, v.le dell'Università n 2, I-35020, Legnaro (Padova) Italy

${ }^{13}$ Dipartimento di Ingegneria Civile, Ambientale, del Territorio, Edile e di Chimica (DICATECh) Politecnico di Bari, Bari, Italy

${ }^{14}$ LAPLACE, Université de Toulouse, CNRS, Toulouse, France

${ }^{15}$ Space Science \& Astrobiology Division, NASA Ames Research Center, Moett Field, CA 94035, USA

${ }^{16}$ Institut des Sciences Moléculaires d'Orsay (ISMO), CNRS - Université Paris-Sud, 91405 Orsay, France

${ }^{17}$ Organization for Research Initiatives and Development, Doshisha University, Kyoto 602-8580, Japan

${ }^{18}$ Physics and Astronomy Dept. "G. Galilei” \& CNISM, INFN, University of Padova, Padova, Italy

${ }^{19}$ Iter Organization (IO), St Paul Lez Durance; France

Received 1 April 2021 / Accepted ?? (C) The Author(s) 2021

\begin{abstract}
This topical review gathers the last updates concerning caesium-free negative ion sources presented during the $63^{\text {rd }}$ Course of the International school of Quantum Electronics of the Ettore Majorana Foundation and European collaborative works related to these lectures. Hence, beyond the frame of this course this topical review addresses both theoretical and experimental work performed during these last few years and complexities represented by the conception of a negative ion source ranging from the creation of negative ions to their neutralization.
\end{abstract}




\section{Introduction}

Since the tandem Van-de-Graaf accelerators in the 1950s [1] negative ion sources (NIS) never ceased to expand into numerous applications with a continuous improvement of their efficiency. In particular, during the past decade NIS have been implanted in fusion applications [2], high energy linear particle accelerators [3], neutron generation [4], tandem accelerators and in accelerator-based mass spectrometry [5].

In fusion applications, plasma sources are a key component of Neutral Beam Injectors (NBI), which consist of a NIS, a neutralizer and a beam conditioner [6]. In modern NBIs, ion source design is based on the so-called tandem configuration where coupling and diffusion zones are magnetically separated. The magnetic filter, uniform magnetic field created by permanent magnets $(\sim 10 \mathrm{mT})$ perpendicularly to the source axis, shields the diffusion zone from hot electrons coming from the coupling area. Consequently, whilst electron temperature $T_{e}$ in the coupling area, or driver, reaches tens of $\mathrm{eV}$, it plummets to one $\mathrm{eV}$ or even less in the diffusion area where the negative ions are extracted. Magnetizing the electrons also significantly reduces the electron current coextracted with the negative ions. In addition, a low electron temperature is beneficial for the residence time of negative ions before being destroyed by collisions with hot electrons.

The critical parameters for the NBI are:

i) uniform negative ion beamlet current density due to the acceptance of the accelerator (divergence of the beamlets lower than $7 \mathrm{mrad}$ );

ii) less than one electron co-extracted per negative ion in $\mathrm{D}_{2}$ and less than 0.5 in $\mathrm{H}_{2}$;

iii) for ITER: $46 \mathrm{~A}^{-} \mathrm{H}^{-}$beam at $0.87 \mathrm{MeV}, 40 \mathrm{~A}$ of $\mathrm{D}^{-}$beam at $1 \mathrm{MeV}$, during $1000 \mathrm{~s}$ and $3600 \mathrm{~s}$ respectively and for DEMO pre-industrial reactor: $40 \mathrm{~A}^{-} \mathrm{D}^{-}$beam at $1 \mathrm{MeV}$ during $7200 \mathrm{~s}$ [7].

In modern $\mathrm{kW}$-scale power NISs, inductively RF coupled plasmas (ICP) replaced arc sources based on Kamaboko sources concept developed at JAERI. Those developments on RF ICP-plasmas, aiming at a large-area negative ion source, have been conducted at IPP-Garching since 2003 with a single ICP plasma source (or driver) in the BATMAN (Bavarian Test Machine for Negative Ions) and MANITU (Multi-Ampere Negative Ion Test Unit) test stands; on ITER half-size negative ion sources with RADI and ELISE (Extraction from a Large Ion Source Experiment) with four drivers before ITER full-scale testing on SPIDER (Source for the Production of Ions of Deuterium Extracted from an RF plasma) ion source and MITICA (Megavolt ITER Injector and Concept
Advancement) NBI test bed with height RF drivers. In the MITICA prototype, which is the fullscale prototype of the ITER NBI hosted in Padua (Italia), a low modularity beam source (LMBS) SPIDER-like ion source will be implemented. The source has a length of $1.9 \mathrm{~m}$ (on the beam axis) and transverse dimensions of the grids are $1.6 \mathrm{~m} \times 0.8$ $\mathrm{m}$ (divided in four segments) [6].

In those NIS, negative ions are produced on a caesiated plasma grid (PG) [153] and extracted through 1280 apertures of roughly $16 \mathrm{~mm}$ in diameter. Caesium $-\mathrm{Cs}-$ is added both to:

i) lower the work function of the metal (tungsten) to induce a direct surface production of negative ions;

ii) dwindle the co-extracted electron fraction.

In the light of the results obtained with SPIDER, MITICA will be employed to address any shortcoming which could prevent the NBI from reaching the nominal working conditions for ITER and for DEMO.

However, even if the extracted current densities produced in ELISE ion source are close to the ITER requirements and pilot data obtained in SPIDER at $0.4 \mathrm{~Pa}$ and $400 \mathrm{~kW}$ in $\mathrm{Cs}$-free $\mathrm{H}_{2}$ plasma [7] indicate that this design fits the concept such LMBS ion sources suffer from:

i) electron drifts in front of the extracting grid [133];

ii) low efficiency neutralization of the negative ion beam (in cold gas $\sim 55 \%$ and $>80 \%$ in a high density plasma which is too difficult to implement in high voltage environment [8]);

iii) long shut down for maintenance performed remotely to get rid of the Cs-coating.

In a Cs-free ion source, negative ions are mainly created in plasma volume by dissociative attachment (DA) between ro-vibrationally excited molecules and cold electrons. These excited molecules can be created either in plasma volume (direct excitation) or on surfaces (recombinative desorption). Negative ions can be also created directly on surfaces from positive ions. However, up to now, neither a Cs-free NBI or a drift less NBI which could meet the ITER and DEMO requirements in terms of extracted current and duration exists.

In this paper, we address several aspects through new improvements in experimental systems and numerical methods which could lead to the design of an alternative Cs-free NBI system which could: i) reduce the electron drift in front of the plasma electrode; ii) increase the wall-plug efficiency (ratio of the neutral power at the entrance of the tokamak and the total electric power consumed in the injector: ion source, accelerator, recovery 
electrode, auxiliary systems) of the NBI which remains lower than $30 \%$ [8]; iii) eliminate regular cleaning and maintenance of the ion source which should be done via remote handling.

For that purpose, the paper is organized in three sections (in addition to the introduction section). Section 2 addresses the problem of caesium seeding in $\mathrm{H}_{2} / \mathrm{D}_{2}$ plasmas and how the production of negative ions could be improved in Cs-free plasma by other volume and surface mechanisms. Even though most of the experimental and numerical methodologies have been introduced in the late ' $80 \mathrm{~s}$ and in the ' $90 \mathrm{~s}$, new improvements in experimental set-ups give the possibility to scrutinize fundamental volume and surface mechanisms which enhance indirectly or directly the volume production of negative ions by DA. Section 3 highlights some new concepts of plasma sources (functioning and last experimental results) to produce negative ions in Cs-free plasmas and possible options to improve negative ion production in existing setups. These new designs of plasma sources represent breakthroughs in the tandem concept: the same magnetic field shields the diffusion zone from hot electrons and ensures the power coupling with the plasma. These high aspect ratio (height over width) plasma sources similar to the high modularity beam source (HMBS) proposed for DEMO [6] could improve the wall-plug efficiency by offering the possibility to employ laser photoneutralization instead of gaz or plasma. Nevertheless, improvement for an NBI should be global and a dedicated plasma chamber equipped with plasma diagnostics stresses optimization issues of such a complex device. Section 4 addresses open questions in NIS physics and shows the latest developments in NIS modeling. In the past five years a significant effort has been devoted to model the plasma behavior in NIS by kinetic particle-based models and to control the full source by the application of complex network theory. The first have been optimized to run on supercomputers via a parallelization of the algorithms using MPI and OpenMP libraries and will be presented in Sect. 4.1, while the latter is explained in detail in Sect. 4.2.

\section{Fundamental approach of the negative ion production in a Cs-free plasma}

Many research projects in the negative ion community have been devoted to finding alternative solutions to the use of caesium. The latter may diffuse outside of the ion source and induce secondary particle production or voltage breakdowns inside the accelerator vessel requiring a regular maintenance in a nuclear environment. Possible solutions for instance would be:

(1) to replace the ion source wall materials by a type with a lower work function;

(2) to modify the geometry of the device so as to increase the electron confinement and as a result the negative ion yield produced in volume by DA of the hydrogen isotope molecules. In this case, new difficulties related to the extraction across the plasma sheath could be introduced.

Both solutions have been implemented in different source prototypes (see Sect. 3), while here the relevant mechanisms to produce vibrationally excited molecules (precursors of negative ions) in volume (Section 2.1) and on surfaces (Section 2.2) are discussed.

\subsection{Global models and relevant elementary processes in caesium-free NIS: early studies}

A large effort has been made to develop state-tostate non-equilibrium vibrational kinetics coupled to the electron energy distribution function for NIS modeling. The first attempt in this direction was made in the seventies by the group operating at the University of Bari in collaboration with the Center of Plasma Chemistry (CNR) with the publication of two papers dealing with the dissociation of $\mathrm{H}_{2}$ in cold plasmas $[9,10]$. The first [9] studied the state-to-state vibrational distribution of $\mathrm{H}_{2}$ demonstrating the possibility that a pure vibrational mechanism could compete with electron impact dissociation of $\mathrm{H}_{2}$, while the second [10] introduced for the first time the coupling of the vibrational distribution function (VDF) and the electron energy distribution function (EEDF) also proposing the exploration of classical approaches (in particular the Gryzinskii approach) to obtain state-resolved electron impact dissociation cross sections.

In the same years Bacal and Hamilton [11] observed the formation of $\mathrm{H}^{-}$and $\mathrm{D}^{-}$during experiments in non-equilibrium plasmas. This work was very important because it proved the negative ion production, while at that time the international community was convinced that only the production of positive ions $\left(\mathrm{H}_{2}^{+}, \mathrm{H}^{+}\right)$was possible in $H_{2}$ plasmas. Soon Hiskes [12-15] tried to explain the formation of negative ions as due to the electron dissociative attachment (DA) process initiated from vibrationally excited $\mathrm{H}_{2}(v)$ levels, whose cross sections were calculated by Bardsley and Wadhera et al. [16-18]. The knowledge of cross sections can be considered as the basic ingredient for NIS modeling: Bretagne et al [19] and Gorse et al [20-22] developed a self-consistent code for the calculation of the EEDF in the 
magnetic multicusp NIS operating at the École Polytechnique. The EEDF code was then coupled to the non-equilibrium VDF [18] accounting for the mechanism of resonant vibrational excitation induced by low-energy electrons (process represented as $\mathrm{eV}$ ) and also the so-called EV processes, i.e. the indirect vibrational excitation by radiative cascade from singlet states following the excitations by impact of high-energy electrons. The modeling of the onset of the vibrational nonequilibrium in the $\mathrm{H}_{2}$ distribution in NIS was the key for the accurate description of the mechanism of formation of negative ions [23]. The EV processes were recognized to contribute significantly to the population of the tail of the $\mathrm{H}_{2}$ VDF and the corresponding cross sections initiated from the $v_{i}=0$ vibrational level were estimated at first by Hiskes et al [12], identifying the $B^{1} \Sigma_{u}^{+}$and $C^{1} \Pi_{u}$ states as the main contributors and the lowlying Rydberg singlets belonging to the same symmetries accounting for the remaining fraction. The implementation of a detailed kinetic scheme went through the construction of a complete database of state-to-state cross sections also considering the role of the dissociative radiative decay processes, relevant for the vibrational levels lying close the dissociation limit. These results for transitions promoted by vibrationally excited molecules were obtained by Celiberto et al $[18,24,25]$ exploiting the semi-classical impact parameter method (IPM) and allowed also the investigation on the existence of an isotopic effect. Recently a complete revision of the database of electron-impact induced processes has been carried out in the framework of the convergent closecoupling (CCC) formalism. It represents the most accurate and up-to-date dynamical results [26].
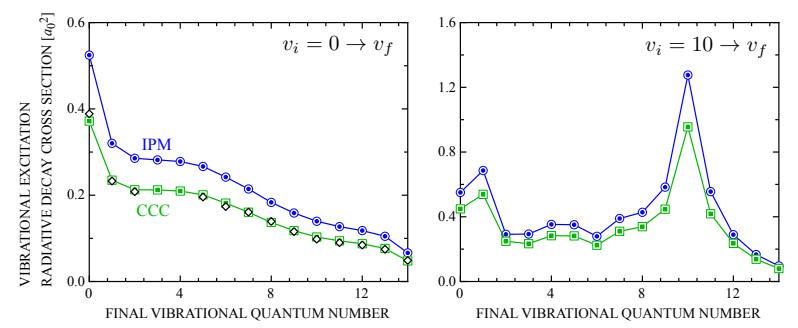

Fig. 1 Distribution of vibrational excitation radiative decay EV cross sections versus final vibrational quantum number for excitations from the $v_{i}=0$ and 10 at the collision energy $E=50 \mathrm{eV}$. (circles) IPM approach $[24,18]$, (squares) CCC method [26], (diamonds) Hiskes results [27].

In Fig. 1a comparison between IPM and CCC cross sections is displayed. The distribution versus the final vibrational levels at $50 \mathrm{eV}$ of the collision energy (a value close to the maximum of cross sections) for excitations starting from $v_{i}=0$ and 10 are shown, the CCC results including all the relevant radiative singlets in the spectrum, while the IPM only the four principal contributors, i.e. $B, B^{\prime}, C$ and $D$ singlet states. The agreement of the CCC results with those by Hiskes [27] for the $v_{i}=$ $0 \rightarrow v_{f}$ excitations is striking, while the IPM approach overestimates the cross sections. It is worth noting that the discrepancy remains within a factor two and is even compressed when initial vibrational excited levels are considered, as shown for $v_{i}=10$. The satisfactory agreement found tells about the agreement of vibronic excitations to excited singlet states and the same general considerations can be extended to dissociative excitation processes induced by electron-impact (a thorough comparison can be found in Refs. $[28,29])$, thus confirming that the semi-classical approach could be a reasonable choice for nonresonant excitations in dipole-allowed transitions. In fact, also the $H_{2}$ dissociation by electron impact is of great importance in any vibrational kinetic scheme, being the process responsible for destroying the vibrational content of molecules on one hand and possibly leading to the production of excited atomic species on the other. Actually, the dissociation in $\mathrm{H}_{2}$ plasmas proceeds mainly through the excitation of triplet states, the purely repulsive $b^{3} \Sigma_{u}^{+}$and the $a^{3} \Sigma_{g}^{+}$and $c^{3} \Pi_{u}$ states with direct and indirect mechanisms, respectively [30]. In particular for the excitation to the $b^{3} \Sigma_{u}^{+}$state different vibrational-specific results have been obtained in the past in the framework of different theories, namely the classical Gryzinski [18], quantum R-matrix [31,32] and complex Kohn variational [33] approaches, that showed a general agreement also, in the case of $v_{i}=0$, with accurate electron energy-loss spectroscopy (EELS) measurements [34-36] (see for a detailed comparison Ref. [37]). The CCC results, however, predict a different position of the maximum with an absolute value a factor two smaller and triggered new experiments with a transmission-free time-offlight electron spectrometer eventually finding an excellent agreement [38].

The resonant processes entail both dissociative attachment and vibrational excitation through the negative ion formation. The cross sections for the relevant resonances at $3.6 \mathrm{eV}\left(X^{2} \Sigma_{u}^{+}\right), 10 \mathrm{eV}$ $\left(B^{2} \Sigma_{g}^{+}\right)$and $14 \mathrm{eV}$ (the Rydberg ${ }^{2} \Sigma_{g}^{+}$state) have been deeply investigated in the past [39-42,18]. However, a problem centered on the role of dissociative attachment from Rydberg states of $\mathrm{H}_{2}$ molecule, lying close to the $\mathrm{H}_{2}^{+}$ground state, in the mechanism of $\mathrm{H}^{-}$formation, is still open in the 
literature. In fact, Pinnaduwage et al [43] reported for these states, rates several orders of magnitude higher than the corresponding rates involving the electronic ground state of $\mathrm{H}_{2}$. The inclusion of these processes in the vibrational kinetics of $\mathrm{H}_{2}$ significantly contributes to the negative ion formation [44] differently from the previous results obtained [13].

In the kinetic scheme, at the same level of relevance as electron-impact induced processes, it should be considered the inclusion of rate coefficients for energy transfer between vibrational and translational degrees of freedom (VT processes) in atom-molecule collisions, responsible for an efficient mechanism of vibrational deactivation. Esposito et al $[45,46]$ extensively used the quasi-classical (QCT) dynamical approach to calculate complete sets of cross sections and rate coefficients for $\mathrm{H}+\mathrm{H}_{2}(v)$ collisions. Those works include results for VT processes and collisional-induced dissociation and recombination. The study of the reliability of QCT dynamics including hydrogen species has been recently treated in some papers [47-50]. The VT processes of $H+H_{2}(v)$ can be thought as the sum of three different contributions, the purely inelastic, the quasi-reactive and the reactive ones (see details in [49]). At low energy, the purely inelastic process as obtained by QCT can be affected by significant error (it is a "classically forbidden" process, as defined by W. H. Miller [51]). However, even for $v=1$, the $H+H_{2}$ system is energetically above the reaction barrier. This means that the quasi-reactive and reactive processes are active even for the lowest non-null vibration. They are not subject to the purely inelastic issue in QCT, and give an important contribution to the total VT rates, even at low energy. They can be in serious error only for the presence of significant tunneling through the reaction barrier, but above the barrier even this effect should be of limited impact. This explains why QCT results for $H+H_{2}\left(v=1 \rightarrow v^{\prime}=0\right)$ in $[45,46]$ are in good agreement with the most recent experimental results [51]. More complex is the condition for dissociation, but even in that case a correct QCT treatment including both direct and indirect dynamics [46] can yield results in good agreement with experimental results and with quantum mechanical methods [52].
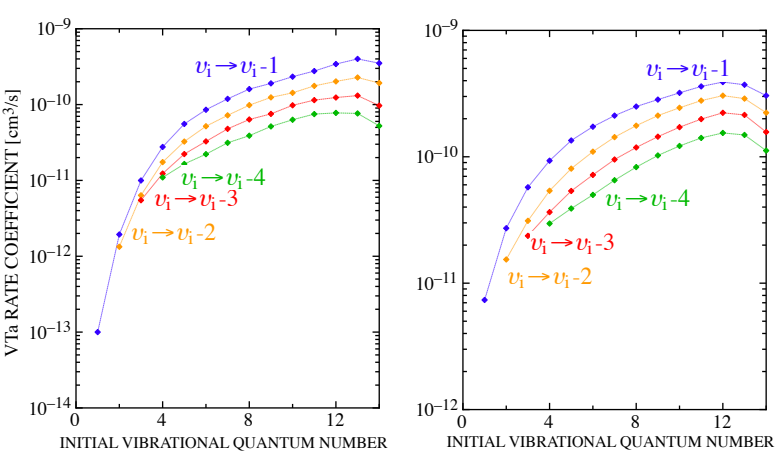

Fig. 2 VT rates in $\mathrm{H}-\mathrm{H}_{2}(v)$ collisions for mono- and multi-quantum de-excitations at $T_{g}=300 \mathrm{~K}$ (left) and $1000 \mathrm{~K}$ (right) [46].

In Fig. 2 the mono- and multi-quantum VT rates are reported for specific values of temperature, showing that, as expected, the probability of energy transfer significantly decreases with increasing the number of vibrational quanta exchanged during the collision. All these processes play a role in shaping the vdf.

Another important point developed at the beginning of NIS modeling was the definition of the role of heterogeneous reactions at the walls of the reactor in redistributing the vibrational energy of excited molecules impinging the wall, a problem investigated theoretically by Hiskes and Karo [15] using a classical method. Very important experimental results on the interaction of atomic hydrogen impinging the reactor walls were reported by Hall et al [53] and by Eenshuistra et al [54].

These studies, showing that in the catalytic recombination of atomic hydrogen at the surface, vibrationally excited $\mathrm{H}_{2}$ states were formed, triggered experiments aimed at identifying the catalyticity of different surfaces in order to enhance the formation of vibrationally excited molecules in the plasma and in turn to increase the negative ion yield [55]. Moreover, the heterogeneous mechanisms of recombination were theoretically studied by Cacciatore and Rutigliano [56-59] in the framework of a semi-classical approach, deriving the probability of recombination on different sites of graphite and metal surfaces, the nascent vibrational distribution of newly formed molecules desorbed from the surface, the probability of competing processes and also investigating the existence of isotopic effects. It is worth noticing that in Ref. [61] a Reduced Linear Model (RLM) of vibrational kinetics has been developed, enabling a straightforward analytic solution of the VDF accounting for vibrational deactivation at the wall.

Many other authors [60-64] have developed similar approaches for a better understanding of the 
formation of negative $\mathrm{H}^{-}$and $\mathrm{D}^{-}$in different sources.

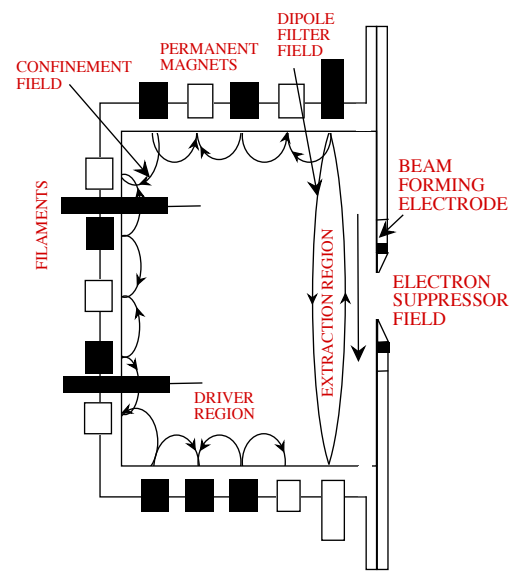

Fig. 3 Scheme of a Magnetic Multicusp Negative Ion source. Reproduced from Ref. [48].

In Fig. 3 the schematic of the magnetic multicusp source, composed of a cylindrical chamber in which the discharge is produced by thermionic electrons emitted by a filament and accelerated up to $100 \mathrm{eV}$, is reported. The accelerated electrons are then degraded, i.e. loose energy in inelastic collisions with the $\mathrm{H}_{2}$ molecules forming an EEDF basically composed of a high-energy long plateau, extending up to $100 \mathrm{eV}$, and by a low-energy Maxwellian portion. This kind of distribution in principle is able to enhance the negative ion production due to the synergic action of:

1) the high-energy electrons (from the high-energy plateau), that form through the EV processes a corresponding plateau in the VDF of $\mathrm{H}_{2}$ molecules; 2) the low-energy electrons, that act on the plateau of the VDF forming negative ions through dissociative attachment.

This scheme strongly depends on the exponential increase of dissociative attachment rates with vibrational quantum number of $\mathrm{H}_{2}$ and $\mathrm{D}_{2}$ molecules [18]. Of course, the kinetic scheme is much more complicated as can be understood from Eqs. (1)-(2), which present the equations for VDF and EEDF in a compact implicit form [1921,61,65]:

$$
\begin{aligned}
& \left(\frac{d N_{v}}{d t}\right)=\left(\frac{d N_{v}}{d t}\right)_{e V}+\left(\frac{d N_{v}}{d t}\right)_{E V}+\left(\frac{d N_{v}}{d t}\right)_{V V}+ \\
& +\left(\frac{d N_{v}}{d t}\right)_{V T}+\left(\frac{d N_{v}}{d t}\right)_{e D}+\left(\frac{d N_{v}}{d t}\right)_{e I}+ \\
& +\left(\frac{d N_{v}}{d t}\right)_{D A}+\left(\frac{d N_{v}}{d t}\right)_{e E}+\left(\frac{d N_{v}}{d t}\right)_{\text {wall }}
\end{aligned}
$$

$$
\begin{gathered}
\left(\frac{\partial n(\varepsilon, t)}{\partial t}\right)=\left(\frac{\partial J_{e l}}{\partial \varepsilon}\right)_{e M}-\left(\frac{\partial J_{e l}}{\partial \varepsilon}\right)_{e e}+i n+ \\
+i o n+\sup +S-L .
\end{gathered}
$$

The different terms in Eq. (1) refer to the different collisional processes shaping the distribution, i.e. the $e V$ and $E V$ processes, previously discussed, the $V V$ (vibration-vibration) and $V T$ (vibrationtranslation) energy exchange processes, the electron impact dissociation and ionization processes $e D$ and $e I$, the dissociative attachment process $D A$, and the electronic excitation $e E$, including the EV processes. Finally, the wall term includes all catalytic processes affecting the VDF. Eq. (2) contains elastic electron terms $(e M)$, electron-electron (ee), inelastic (in), ionization (ion), superelastic (sup) collisions, as well as a source $(S)$ and loss terms $(L)$. Explicit equations for these terms can be found in Refs. [21,61,65].

Basically, this approach, developed by Gorse et al [20], was validated at first qualitatively by the experimental distributions by Eenshuistra et al [66], and later by the good agreement between the theoretical vibrational distributions of Pagano et al [61] and the corresponding values measured by Mosbach [60], as can be appreciated in Fig. 4.
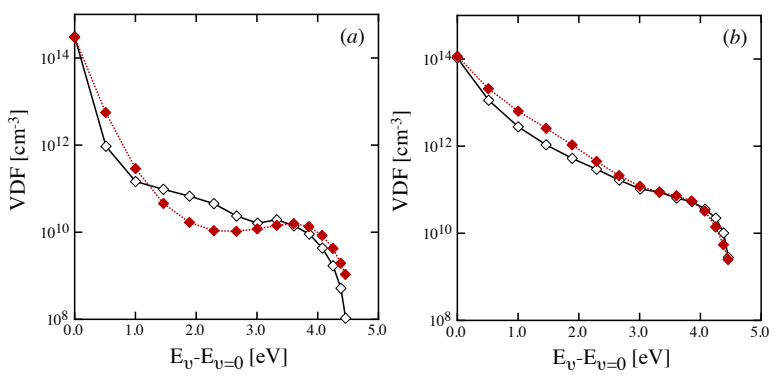

Fig. 4 Comparison of stationary VDF calculated by [61] (open diamonds) and experimental results by Mosbach [62] (closed diamonds) for (a) $I_{d}=0.5 \mathrm{~A}$ and $(b) I_{d}=10$ A. Reproduced from Ref. [48].

Fig. 5 reports a comparison of negative ion concentrations calculated by Pagano et al [63] and the corresponding experimental values of Mosbach [62] showing in general a satisfactory agreement, though increasing the discharge current $I_{d}$ the model predicts a saturation not observed in the experiments that could be ascribed to the cross section dataset included in the model for the dissociative attachment mechanism for $\mathrm{H}^{-}$ production. 


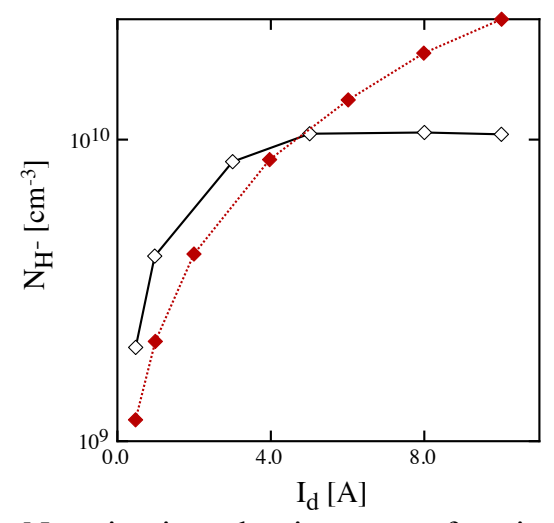

Fig. 5 Negative-ion density as a function of the discharge current for $P=1.5 \mathrm{~Pa}, V_{d}=100 \mathrm{~V}$, calculated by Pagano at al [61] (open diamonds) and experimental results by Mosbach [60] (closed diamonds). Reproduced from Ref. [61].

This approach for the calculation of EEDF was confirmed by Bretagne et al [69] by comparing theoretical (Eq. (2)) and experimental (obtained by using a sophisticated Langmuir probe) values. To close this section in Fig. $6 \mathrm{VDF}$ and EEDF calculated for $\mathrm{H}_{2}$ and $D_{2}$ plasmas [70] have been reported. The EEDF shows the typical profile of the degradation spectrum of an electron beam. The peak is due to the high-energy electrons emitted by the filament and subsequently undergoing collisions with $\mathrm{H}_{2}$ molecules in the plasma, generating the long plateau and the low-energy bulk electrons. The oscillations in the plateau results from the electron energy losses, due to threshold inelastic processes promoted in molecules, and the electron-electron collisions, smoothing the profile in the collisional thermalization of the EEDF. Similar results were reported by Skinner et al [71].
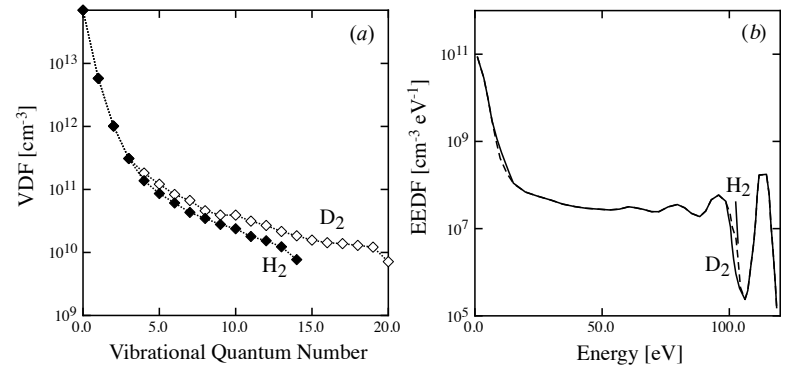

Fig. 6 Theoretical a) vibrational VDF and b) electron energy distribution functions EEDF in $D_{2}$ (full line, open diamonds) and $\mathrm{H}_{2}$ (dashed line, closed diamonds) plasmas; electrical conditions: pressure 4.5 mTorr; current intensity $I_{d}=10 \mathrm{~A}$; applied voltage $V_{d}=115 \mathrm{~V}$; plasma potential $V_{p}=2.9 \mathrm{~V}$. Reproduced from Ref. [68].

It is worth noting that the EEDF follows the lines discussed previously, showing also that the corresponding EEDF for $D_{2}$ plasmas are practically the same as in $\mathrm{H}_{2}$ plasmas. On the other hand, VDF for the two cases show some differences because the number of vibrational levels for $D_{2}$ exceeds the number of levels of $\mathrm{H}_{2}$.

It should be noted that this section does not consider other important aspects of negative ion sources, such as the isotopic effect, the role of the wall and the effect of wall material, the role of the magnetic filter, which will be extensively considered in other parts of the manuscript including the reviews by Bacal and Wada $[72,73,74]$.

\subsection{Experimental investigation on recombinative desorption mechanism in an ECR (2.45 GHz) plasma}

2.2.1 Dipolar plasma source to scrutinize the production of ro-vibrationally excited molecules at lower scale

Actual neutral beam injector (NBI) design shows a global efficiency below $50 \%$ (The total or global efficiency of the injector, called Wall-Plug efficiency, is the ratio of the neutral power at the entrance of the tokamak and the total electric power consumed in the injector (ion source + accelerator + recovery electrode + auxiliary systems). A higher aspect ratio (height over width) could increase the efficiency to more than $60 \%$ [72]. In particular the photoneutralization could be employed on bladelike beams. To meet this requirement, of high aspect ratio, a homogeneous column of $\mathrm{H}_{2}\left(\mathrm{D}_{2}\right)$ plasma of more than one-meter long is needed. In the RAID reactor (Swiss plasma center SPCLausanne) a helicon antenna (bird-cage type) has proven its ability to produce, at $0.3 \mathrm{~Pa}$ and $5 \mathrm{~kW}$, a negative ion density of $2 \times 10^{16} \mathrm{~m}^{-3}$ and $1.75 \times 10^{16} \mathrm{~m}^{-}$ ${ }^{3}$ in $\mathrm{H}_{2}$ and $\mathrm{D}_{2}$ Cs-free plasmas [72] respectively. Similar density of $\mathrm{H}^{-}\left(3.5 \times 10^{16} \mathrm{~m}^{-3}\right)$ is obtained in the BATMAN ion source with an input power of $40 \mathrm{~kW}$ and cesium seeding [73]. Hence, a helicon antenna, as the one set in the RAID reactor, could fulfill requirements for a high efficiency NBI Csfree device. However, the helicon antenna and its magnetic coils which are mandatory to create a homogeneous magnetic field on the reactor axis where the helicon wave propagates are too large to be moved easily to perform diagnostics on the plasma on locations other than EPFL. Nevertheless, plasma obtained with an ECR (electron cyclotron resonance) dipolar plasma source [74] is quite similar to the plasma produced by the helicon antenna in the RAID reactor. 


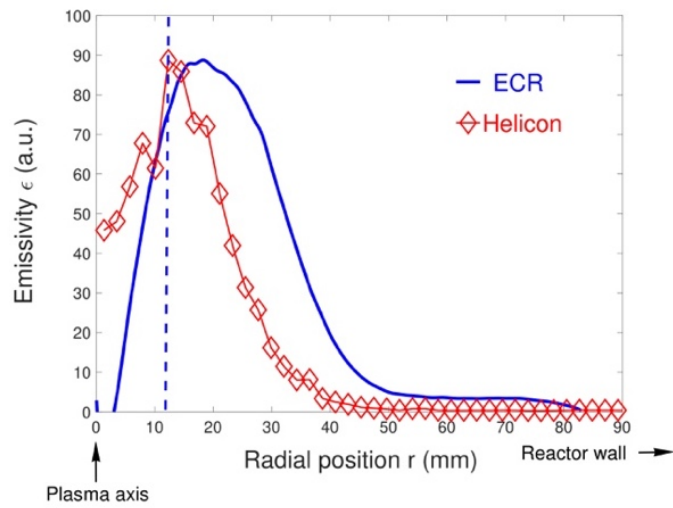

Fig. 7 Emissivity profiles obtained perpendicularly: i) across the mid-plan of the permanent magnet of the ECR source (pure argon, 0.3 Pa, $150 \mathrm{~W}$ ); ii) of the helicon plasma column in the RAID reactor (pure $\mathrm{H}_{2}, 0.3 \mathrm{~Pa}$ and $5000 \mathrm{~W})$; reproduced from [75].

In the cylindrically symmetric case, the light observed in the side-on direction can be used to obtain through the Abel's Inversion the radial emissivity measurements. The plasma sustained by the helicon wave in the RAID reactor and around an ECR plasma source fulfilled this requirement. Hence, we used Abel's inversion of optical emission spectroscopy (OES) measurements on the magnet mid-plan of the ECR source and on the helicon plasma column in the RAID reactor at two wavelengths to get the radial emissivity. Results are presented in Fig. 7. In both cases, we notice that the highest intensity of excited species is located 20 $\mathrm{mm}$ from the plasma axis and that beyond $50 \mathrm{~mm}$ the plasma emissivity nearly vanishes. Radiative life-times of these excited species are only tens of nano-seconds $\left(\tau^{\mathrm{H}}{ }_{3 \rightarrow 2}=10 \mathrm{~ns}\right.$ and $\left.\tau^{\mathrm{Ar}}{ }_{2 \mathrm{P}}{ }^{1}=22 \mathrm{~ns}\right)$, hence these emissivity measurements depict with an accuracy better than $0.5 \mathrm{~mm}$ (distance crossed by an $\mathrm{H}$ atom of $0.5 \mathrm{eV}$ during $50 \mathrm{~ns}$ ) the position where hot electrons collide with neutral species. These results also demonstrate the existence of hot electrons of energy $\left(T_{e}\right)$ equal or above $10 \mathrm{eV}$, statement justified by the energy threshold for these excited states which are $12.08 \mathrm{eV}$ and 13.47 $\mathrm{eV}$ for $\mathrm{H}^{*}(\mathrm{n}=3)$ and $\mathrm{Ar}^{*}\left(2 \mathrm{p}^{1}\right)$, respectively.

When these areas are investigated with a Langmuir probe, see Fig. 8, radial variations of the electron temperature are quite similar when positions ranging from $\mathrm{r}=25 \mathrm{~mm}$ to $\mathrm{r}=53 \mathrm{~mm}$ (from the plasma axis) are considered. The average energy of the EEPF (electron energy probability function), currently named electron temperature $\left(T_{e}\right)$, decreases from $5.5 \mathrm{eV}$ down to $3.8 \mathrm{eV}$ and from 5.2 $\mathrm{eV}$ down to $2.4 \mathrm{eV}$ for ECR and Helicon plasmas, respectively. For electron densities, $\mathrm{n}_{\mathrm{e}}$, obtained by integration of the EEPF, we observe a decrease from $0.16 \times 10^{16} \mathrm{~m}^{-3}$ down to $0.05 \times 10^{16} \mathrm{~m}^{-3}$ and from $0.35 \times 10^{16} \mathrm{~m}^{-3}$ down to $0.04 \times 10^{16} \mathrm{~m}^{-3}$ for
ECR and helicon plasma, respectively, for radial positions ranging from 25 to $53 \mathrm{~mm}$ from the plasma axis.

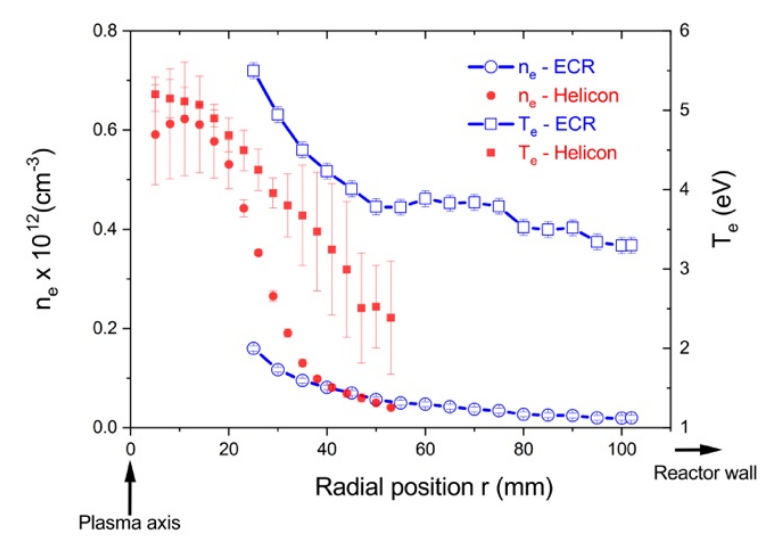

Fig. 8 Radial profiles of $n_{e}$ and $T_{e}$ obtained across: i) the mid-plan of the permanent magnet of the ECR source (pure argon, 0.3 Pa, $150 \mathrm{~W}$ ); ii) across the plasma column in the RAID reactor (pure $\mathrm{H}_{2}, 0.3 \mathrm{~Pa}$ and 3000 W). Reproduced from [72].

Plasma density observed at $25 \mathrm{~mm}$ from the plasma axis are different but the input power also differs by a factor of 20 . If the $n_{i}-n_{e}$ density ratio is considered, results obtained at similar plasma conditions and position in PROMETHEUS-I reactor (see Sect. 3.1) [64] (a network of five identical ECR dipolar sources) and in the RAID reactor [72], are comparable: 0.18 and 0.225 , respectively. In consequence, the plasma features, produced by an ECR dipolar source, can be considered similar enough to the one obtained in the RAID reactor to allow an investigation of the ECR plasma rather than the helicon plasma when plasma diagnostic cannot be set up at SPCLausanne.

\subsubsection{Production of negative ions by $D A$}

The results obtained in the RAID reactor at EPFL $[72,75]$ demonstrate, at full-scale, the efficiency of dissociative electron attachment (DA) to produce a large density of negative ions in Cs-free plasmas. The yield of DA strongly depends on the vibrational level of the ground state molecules [18]: the cross-section increases from $3.11 \times 10^{-28}$ to $1.08 \times 10^{-21} \mathrm{~m}^{2}$ for increasing vibrational level from $\mathrm{v} "=0$ to 6 . These ro-vibrationally excited molecules can be produced both by inelastic collisions, with hot electrons in the plasma volume, and by surface reactions when atoms impinge on a surface and react with already adsorbed atoms. For surface reactions, involved mechanisms are known as Langmuir-Hinshelwood, Eley-Rideal and Hotatom [56]. A large number of theoretical and experimental studies have been published in particular for graphite, tungsten and tantalum [56- 
59,76]. In consequence, as the vibrational levels above $\mathrm{v} "=6$ are essential for the DA reaction it is of interest to develop a reliable and accurate experimental method to measure the absolute vdf.

\subsubsection{VUV spectroscopy on the DESIRS beam} line for the direct measurement of $\mathrm{H}_{2}\left(\mathrm{D}_{2}\right) \mathrm{VDF}$. We present hereafter evolutions of the SCHEME (Source of exCited HydrogEn MolEcules) setup, see Fig. 9, and the methods of measurement deployed at SOLEIL synchrotron on the DESIRS (Dichroïsme Et Spectroscopie par Interaction avec le Rayonnement Synchrotron) [77] beam line over the past two years to investigate the recombinative desorption of $\mathrm{H}$ - and $\mathrm{D}$-atoms of surfaces of different materials. In these setups, the same plasma source is employed (ECR plasma source, plasma OFF in Fig. 9a and plasma ON in Fig. 9b). Throughout this document we will use the following notation for concision: BX02 (or BX 02) describes, for instance, the transition $\mathrm{B}(\mathrm{v} "=0) \leftarrow \mathrm{X}(\mathrm{v} "=2)$.

\subsubsection{VUV-LIF and VUV absorption in cold} gas.

In a first attempt, heated filaments were used instead of a plasma source to avoid the saturation of the photomultiplier tube (PMT) by photons coming from the plasma at the same energy. Gabriel et al. [78] have successfully employed VUV-LIF (light induced fluorescence in the vacuum ultra-violet) to measure in $\mathrm{H}_{2}$ plasma rovibrational levels up to $\mathrm{v} "=8$. The SCHEME-I [79] setup has been installed on the B-branch endstation of the DESIRS monochromatic beam line.
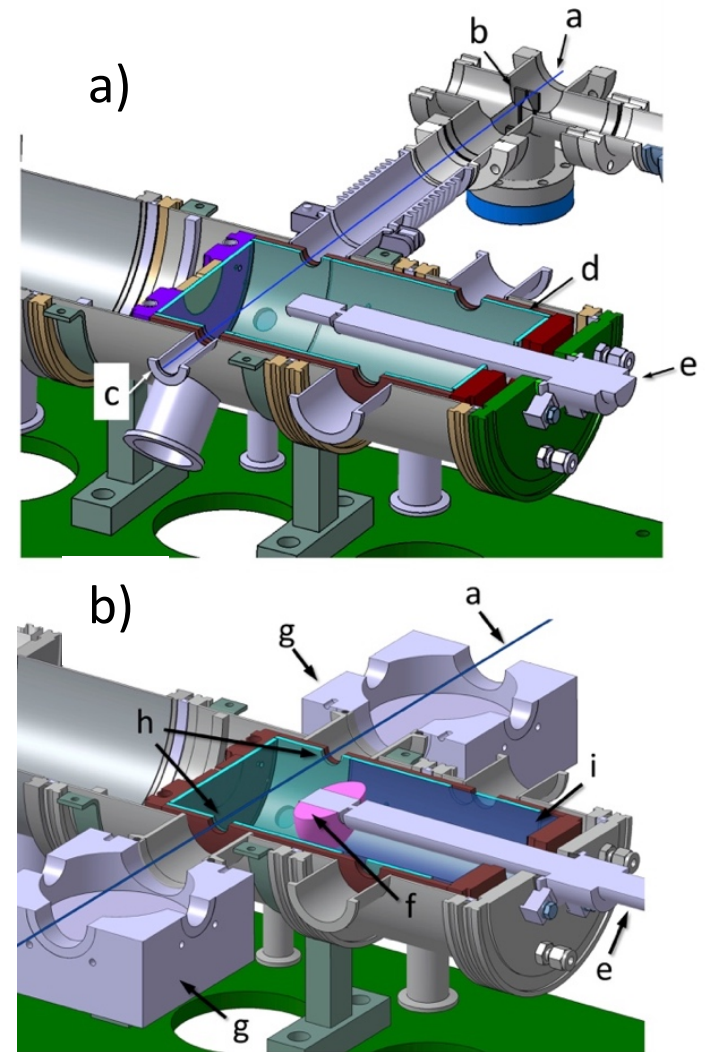

Fig. 9 Schematics of setups employed on: 9a) B-branch and 9b) FTS-branch. a) SR beam, b) oscillating fork chopper $(130 \mathrm{~Hz}), \mathrm{c})$ position of the solar-blind PMT, d) quartz cylinder housed in a cooled-copper cylinder, e) ECR (2.45 GHz) dipolar plasma source, f) ECR coupling zone, g) differential pumping stages $(\mathrm{x} 2), \mathrm{h})$ positions of diaphragms $\left(3 \times 5 \mathrm{~mm}^{2}\right)$, i) foil of the investigated material.

However, despite the use of an optical system enhancing light collection perpendicularly to the SR beam and of a lock-in amplifier for phasesensitive detection, VUV-LIF signal was more difficult to collect than VUV absorption peaks. Fig. 10 top, presents the absorbance of the ground state excited molecule $\mathrm{H}_{2}\left(\mathrm{v}^{\prime}, \mathrm{J} "\right)$ in its $\mathrm{v}^{\prime \prime}=2(10.419 \mathrm{eV})$ ro-vibrational level, observed at $100 \mathrm{mTorr}^{\mathrm{H}_{2}}$ pressure with a stainless steel surface at $300 \mathrm{~K}$. When filaments were heated up to $2000 \mathrm{~K}$, the increase of the absorbance confirms a higher dissociation of the molecules [80].

\subsubsection{VUV absorption in ECR plasmas}

In the light of this first positive results, the experimental setup was modified to selectively probe in the same way, by monochromatic VUV direct absorption, the rovibrational levels of excited molecules in the electronic ground state $\mathrm{H}_{2} / \mathrm{D}_{2}\left(\mathrm{v}^{\prime}, \mathrm{J}^{\prime \prime}\right)$ created by recombinative desorption of H(D) atoms on the surface of different materials (tantalum, tungsten and Quartz as a bare surface) in $\mathrm{H}_{2}\left(\mathrm{D}_{2}\right)$ plasmas. To increase the resolution, a 2400 grooves. $\mathrm{mm}^{-1}$ grating was used instead of a 200 
grooves.mm ${ }^{-1}$, when filaments were used. With both a monochromator entrance and exit slits set at $50 \mu \mathrm{m}$, the resolution was close to $3 \mathrm{~nm}$. The experimental setup presented in Fig. 9a, SCHEMEII consists of an ECR plasma source which allows easier and broader operating conditions: pressure (0.027 - 11 Pa) and microwave power (10 - $200 \mathrm{~W})$. A solid-state microwave $(2.45 \mathrm{GHz})$ generator and an actively cooled plasma source and plasmafacing surfaces allow highly stable and repeatable working conditions (over more than two hours and from a day to the next). The plasma created in a quartz cylinder, b) in Fig. 9a, is probed by the synchrotron radiation (SR) beam, a) in Fig. 9, going through the plasma diameter. Effect of the investigated material, i) in Fig. 9b, is quantified when comparing absorption obtained with and without the material. This improved setup gave us the possibility to observe the following transitions: $\mathrm{B}-\mathrm{X}\left(\mathrm{v}^{\prime}=0 ; \mathrm{v} "=3,4,5\right.$ and 6$)$. All these transitions were selected on the basis of favorable FranckCondon factors. For each v", 3 to 7 rotational levels were observed that allow to obtain statistics. With this second set of measurements, significative differences have been observed between different plasma-facing materials. Fig. 10 top and middle show how the accuracy of the spectrum has been increased between the two measurements performed with SCHEME-I \& -II setups. With a tantalum foil facing the plasma, the absorbance of the $\mathrm{BX}(04)$ transition is increased by $45 \%$ (as measured on the different rotational transitions) compared to quartz material [79].

\subsubsection{VUV Fourier transform absorption in ECR plasmas}

The Fourier transform spectroscopy (FTS), in the VUV range, only available on the DESIRS FTSbranch allows the use of a wide energy range combined with high resolution, to obtain simultaneously numerous spectroscopic data. The paramount advantage of the FTS is the possibility of recording numerous rotational lines (i.e. at least ten transitions / vibrational level) over a single scan which is critical in absorption spectroscopy.

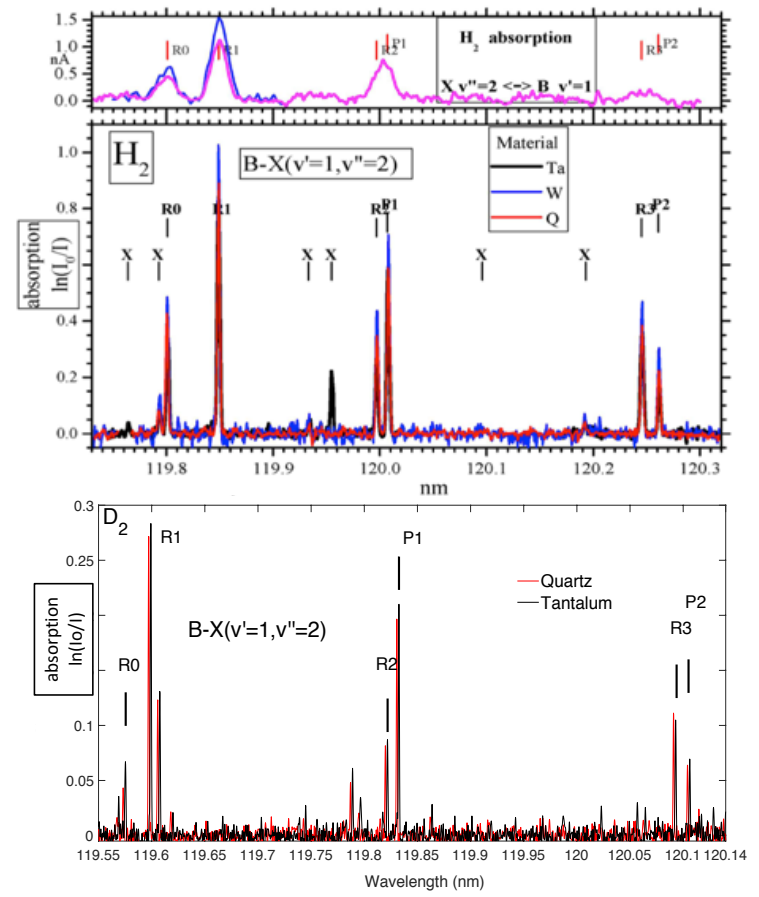

Fig. 10 Absorbance obtained: on the B-branch with heated tungsten filament, $1800 \mathrm{~K}$ (magenta line) and $2000 \mathrm{~K}$ (blue line), $\mathrm{H}_{2}$ cold gas, $13 \mathrm{~Pa}$ (Top); in $\mathrm{H}_{2} \mathrm{ECR}$ plasma at 1.6 $\mathrm{Pa}$ (Center); in $\mathrm{D}_{2}$ ECR plasma at $1.6 \mathrm{~Pa}$ on the FTS-branch (Bottom).

The previous measurements were highly valuable to demonstrate the efficiency of surfaces to enhance the production of ro-vibrational levels. However, levels above $\mathrm{v} "=6$ remained unreachable and the use of a closed cell $\left(\mathrm{MgF}_{2}\right.$ widows $)$ which allowed high pressure functioning in the plasma reactor, was restraining the photon energy domain below $10.196 \mathrm{eV}\left(82237 \mathrm{~cm}^{-1}\right)$. Consequently both Lyman series and $\mathrm{BX}\left(\mathrm{v}^{\prime}, \mathrm{v} "=0\right)$ transitions cannot be investigated to obtain absolute densities of atoms (with the Lyman series) and of the different ro-vibrational levels (with the $\mathrm{BX}\left(\mathrm{v}^{\prime}, 0\right)$ transitions). To circumvent these difficulties, the experimental setup SCHEME-II+ (presented in Fig. 9b) was implemented with two differential pumping stages (entrance/exit of the SR beam) allowing windowless functioning in $\mathrm{H}_{2}$ or $\mathrm{D}_{2}$ gas up to $1.6 \mathrm{~Pa}$, on the DESIRS FTS-branch. With thin $\left(0.1 \mathrm{~mm}\right.$ thick) and small apertures $\left(15 \mathrm{~mm}^{2}\right)$ diaphragms, the gas flow from the reactor to the beam line (entrance and exit of the beam) is drastically decreased. Thanks to these diaphragms, absorption length is also restricted to the reactor diameter with a passive gas contribution (outside the reactor) limited to $\sim 15 \%$ of the total species density. The VUV-FTS [77] coupled to the undulator-based DESIRS SR is able to provide simultaneously a high spectral resolution (maximum resolving power $\mathrm{R}=\lambda / \Delta \lambda \approx 10^{6}$ ), a high accuracy of the energy scale over a large spectral 
range ( $5 \mathrm{eV}$ to $30 \mathrm{eV} \approx 250$ to $40 \mathrm{~nm}$ ) as well as a large flux $\approx 10^{11}$ photons $/ \mathrm{s}$ ). In any case a trade-off must be made between the use of a given high resolution and the duration of one measurement it entails. We have chosen $R=150000$. Fig. 10 bottom shows the improvement obtained on the spectra with the FTS-branch [55] for the same wavelength range observed on the B-branch with different gratings, 200 and 2400 grooves. $\mathrm{mm}^{-1}$, top and bottom of the same figure, respectively.

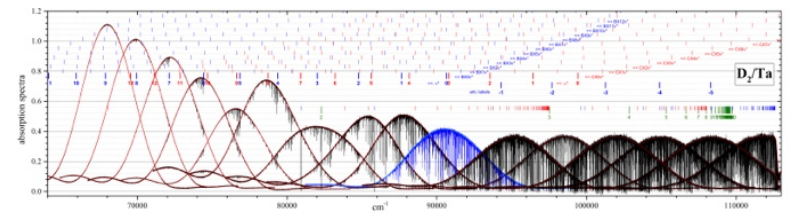

Fig. 11 FTS spectra obtained in $\mathrm{D}_{2}$ plasma $(150 \mathrm{~W}, 1.6$ $\mathrm{Pa}$ ) with a tantalum foil facing the plasma. upper part of the figure: Bandheads (R0) of the observable $\mathrm{D}_{2} \mathrm{BXv}$ 'v" bands (in blue) and CXv'v" ones (in red). The lower line shows in blue and labelled with $\mathrm{v}$ " the chosen settings centered on the BX0v" bandhead values. Shown and labelled in red are the positions of the CX0v" bandheads. Middle part: The small bars correspond to $\mathrm{Ar}, \mathrm{Kr}$ and $\mathrm{Xe}$ lines present as impurities (Ar in black, Xenon in red) or $\mathrm{Kr}$ (in blue) used in the gas filter to eliminate the undulator harmonics. In green is the D Lyman series labelled with the upper n, up to ionization. Lower part: All spectra are shown at a unique scale. Absorptions are observable down to BX09 (to the left) though higher X v" levels are observable for transitions with $v '>0$ at lower energies.

Fig. 11 presents FTS spectra covering all $\mathrm{D}_{2}$ bands between BX00 $\left(\mathrm{v}^{\prime}=0, \mathrm{v} "=0\right)$ and BX09 $\left(\mathrm{v}^{\prime}=0\right.$, $\mathrm{v}$ "=9) when the material facing the plasma is Tantalum in the $64000-113000 \mathrm{~cm}^{-1}$ energy range. Each of the Gaussian like spectra corresponds to a different setting of the undulator. For each, data are useful down to the FWHM. At the selected plasma conditions (1.6 Pa and $150 \mathrm{~W}$ excitation), an obvious number of rotational lines in each vibrational band are saturating at energies higher than $\sim 93000 \mathrm{~cm}^{-1}$. Nevertheless, the saturation affects only those with low J" numbers while they are still available for measurements and comparisons without showing saturation at higher J". In any case, despite the crowded aspect of the figure, there is, while zooming, a clear separation of the lines with just a very low number of overlaps or blends. In addition, as already demonstrated [55], the population of a given v" ground state level results not only from a single measurement but from all other observed BXv'v" transitions to this specific $\mathrm{v}$ " level. The possibility to use different overlaps of undulator settings to observe the same absorption line reinforce the accuracy of our measurements. The undulator settings with negative labels (on Fig. 11) are not only used to reach not saturating high $\mathrm{n}$ numbers of the Lyman series but also to cover non-saturating BXv'0 vibrational bands at high v'. To obviate the saturation problem, we also record the BXv'0 bands at different pressures without plasma.

\subsubsection{Results}

\subsubsection{Results of VUV-FTS for atoms}

The windowless setting allowed the observation of Lyman transitions at higher energies than the commonly used Ly- $\alpha$, namely from Ly- $\gamma$ to Ly- $\eta$ for $\mathrm{H}$ (97.25 and $93.07 \mathrm{~nm}$ respectively). Their oscillators strength are 14 and 86 times lower than the transition of $\mathrm{Ly}-\alpha$, respectively. Hence, measurements at weaker absorbances are allowed avoiding the saturation mostly observed with Ly$\alpha$.

Consequently, knowing the absolute density of $\mathrm{H}(\mathrm{D})$-atoms and the gas temperature (obtained with rotational transitions of the $\mathrm{BX}\left(\mathrm{v}^{\prime}, 0\right)$ levels $)$ it was possible to determine the dissociation degree $D=n_{\text {atom }} /\left(n_{\text {atom }}+2 n_{\text {molecule }}\right) \quad(n$ refers to species density) of molecules in the plasma.

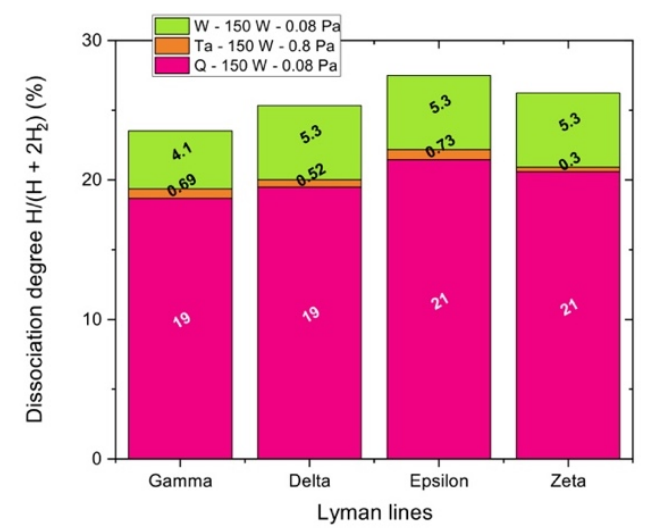

Fig. 12 Dissociation degree obtained with the H Lyman series: Ly- $\gamma$ (97.25 nm), Ly- $\delta$ (94.97 nm), Ly- $\varepsilon$ (93.78 $\mathrm{nm})$, and Ly- $\varsigma(93.07 \mathrm{~nm})$ for different materials and pressures.

Results presented in Fig. 12 show a good accordance between the dissociation degree obtained regardless of the considered spectral line (from Ly- $\gamma$ to Ly- $\zeta$ ). A huge effect of the quartz coverage (recombination coefficient, $\gamma=0.004$ at $290 \mathrm{~K}$ ) on the atom density in the plasma is obvious: the dissociation degree increases, at 0.08 $\mathrm{Pa}$ and $150 \mathrm{~W}$ of microwave intake power, from $5 \%$ when tungsten is the plasma-facing material $\gamma=0.5$ at $240-300 \mathrm{~K}$ ) to $20 \%$ for quartz surface (average values). A significant effect is obtained when the pressure is 10 times higher: with a 
metallic surface of similar recombination coefficient ( $\mathrm{Ta}$ instead of $\mathrm{W}$ ), the dissociation degree plummeted to only $0.6 \%$. Hot electrons produced in the ECR coupling area collide with neutrals and loss a significant quantity of their energies [79]; consequently, with the same intake power, dissociation induced by inelastic collisions is less efficient.

Even if these measurements confirm the role of $\gamma$ played in surface recombination of atoms dissociated by hot electrons, measurements of the VDF is the only possibility to unveil the effect of recombinative desorption on the populations of the rovibrational levels of molecules.

\subsubsection{Absolute $\mathrm{H}_{2}\left(\mathrm{D}_{2}\right)$ molecular densities in the vibrational levels of the ground state}

For pure $\mathrm{H}_{2}\left(\mathrm{D}_{2}\right)$ gas at room temperature in plasma-OFF condition, all populations lie in the ground state $X^{1} \sum_{g}^{+}\left(v^{\prime \prime}=0, J^{\prime \prime}\right)$ levels. Thus, absorption spectra, obtained in open-cell setup, are only composed of BXv'0 lines connected to this level which could be used for population calibrations in combination with the atom density. In plasma-ON condition, every levels of each transition $(\mathrm{BX}, \mathrm{CX}, \mathrm{B}$ 'X, ...) could be populated (v" and $J " \neq 0$ ). Consequently, spectra are much more populated [55] and should be thoroughly processed to obtain VDF for the different materials. Fig. 13 shows a Boltzmann plot of the VDFs obtain at 150 $\mathrm{W}$ and $0.8 \mathrm{~Pa}$ at an identical position in the plasma (40 $\mathrm{mm}$ from the ECR coupling zone, see Fig. $9 \mathrm{~b}$. These VDFs exhibit, below $\mathrm{v} "=3$, the same Boltzmann distribution for all materials whereas the four curves are diverging afterwards. Such a behavior highlights an identical creation mechanism for the first three levels, in the plasma volume, and a creation on the plasma-facing material for higher levels. In particular, tantalum coverage allows VDF to be populated till v" $=10$, whereas Quartz conditions limits the VDF population to $\mathrm{v"} \leq 7$.

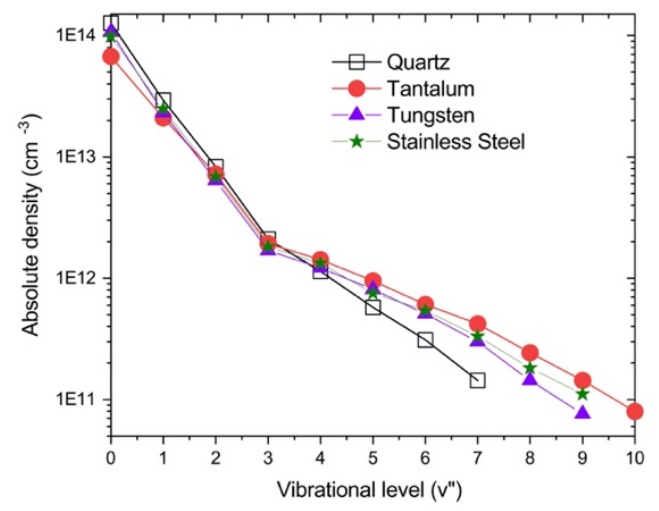

Fig. $13 \mathrm{D}_{2}$ absolute molecular densities $\left(\mathrm{cm}^{-3}\right)$ measured in the plasma core for v" vibrational levels of the electronic ground state levels, at $0.8 \mathrm{~Pa}$ and $150 \mathrm{~W}$ plasma conditions.

Such difference observed between the VDFs, obtained in identical plasma condition, demonstrate the role of the recombinative desorption in the creation of high vibrational levels of the molecule which contribute significantly to the production of negative ions in the plasma by DA mechanism.

\subsection{Study of negative-ion surface production in hydrogen plasma in interaction with a low work-function electride material}

In this Section, ion mass and energy spectrometry analysis are employed to investigate negative ion surface production on a low work-function (WF $\sim 2.4 \mathrm{eV}$ ) conductive ceramic Furthermore, two new diagnostics recently developed are introduced. The first one aims at measuring the absolute flux of surface produced negative ions. The second one aims at measuring in-situ material WF to monitor its possible changes under plasma irradiation.

\subsubsection{Experimental set-up}

Sample materials under study are placed in a lowpressure hydrogen or deuterium plasma (1-2 Pa) [85]. The sample is negatively biased, positive ions from the plasma bombard the surface and negativeions formed upon positive-ion impacts are accelerated from the sample towards the plasma and can be self-extracted to a detector placed in front of the sample. Under the low-pressure conditions considered many NI reach the detector without collisions. Two detectors have been employed in this study. First a mass spectrometer with energy analyzer (Hiden EQP 300) allows measuring $\mathrm{H}^{-}$or $\mathrm{D}^{-}$negative-ion energy distribution functions (NIEDFs). Second a Magnetized Retarding Field Energy Analyzer (MRFEA) allows measuring negative-ion currents. This paper compiles data obtained using ECR (Electron Cyclotron Resonance) or ICP (Inductively Coupled Plasma) plasma sources both described in reference [85]. There is not much difference concerning plasma parameters between both plasma sources. The sample biases are the same (from $0 \mathrm{~V}$ to $-130 \mathrm{~V}$ ), the dominant positive ion in the plasma is identical, $\mathrm{H}_{3}{ }^{+}$or $\mathrm{D}_{3}{ }^{+}$being the most abundant ion, the positive ion flux is around $7 \times 10^{18} \mathrm{~m}^{-2} \mathrm{~s}^{-1}$ in ECR $(1 \mathrm{~Pa}, 60 \mathrm{~W})$ and from $5 \times 10^{18}$ $\mathrm{m}^{-2} \mathrm{~s}^{-1}$ to $5 \times 10^{19} \mathrm{~m}^{-2} \mathrm{~s}^{-1}$ in ICP $(1-2 \mathrm{~Pa} 200 \mathrm{~W}-800 \mathrm{~W})$. $\mathrm{H}_{3}{ }^{+}$or $\mathrm{D}_{3}{ }^{+}$ions dissociate at impact on the surface and their energy is shared between the fragments. So the ion impact energy is defined by the plasma 
potential $\mathrm{V}_{\mathrm{p}}$ and the surface bias $\mathrm{V}_{\mathrm{s}}$ and is equal for most of the impacting ions to

$E=e\left(V_{p}-V_{s}\right) / 3$

Nanoporous $\quad 12 \mathrm{CaO} \cdot 7 \mathrm{Al}_{2} \mathrm{O}_{3} \quad\left(4 \mathrm{e}^{-}\right)$electride (C12A7) [81] samples from Japan have been studied. Electrides are ionic compounds in which some electrons act as anions. The $12 \mathrm{CaO} \cdot 7 \mathrm{Al}_{2} \mathrm{O}_{3}$ $\left(4 \mathrm{e}^{-}\right)$is known as the first air and room temperature stable electride solid material. It is mechanically robust and machinable. The electride crystal structure consists of twelve positively charged cages made of $\mathrm{Ca}, \mathrm{O}$ and $\mathrm{Al}$ and four free electrons, creating a cage conduction band (CCB) at low energy below the vacuum level. This exotic electronic structure gives the electride a low WF of $2.4 \mathrm{eV}$ [82]. Electride samples of about $1 \mathrm{~cm}^{2}$ are positioned in the plasma chamber facing the detector using a four axes manipulator (translation along three orthogonal $\mathrm{X}, \mathrm{Y}, \mathrm{Z}$ axis and rotation around $Z$ axis). Once installed, the sample can be heated-up to $800{ }^{\circ} \mathrm{C}$ and can be biased under plasma irradiation.

\subsubsection{Results and discussion}

\subsubsection{Mass spectrometer analyses}

The experiments presented in this section have been obtained using the ECR source. The mass spectrometer orifice is set at ground and is facing the sample biased at a negative-voltage $V_{s}$. Negative ions formed on the surface gained $-\mathrm{eV}_{\mathrm{s}}$ in kinetic energy between sample and mass spectrometer. The mass spectrometer analyses ions in mass and energy providing the Negative-Ion Energy Distribution Function (NIEDF). For graphical representation, the energy gain $-\mathrm{eV}_{\mathrm{s}}$ is subtracted from the measurement giving an energy scale in which the origin corresponds to a negativeion created at rest on the surface. NIEDFs measured using the $\mathrm{C} 12 \mathrm{~A} 7$ electride material, gadolinium (a low WF metal, $\mathrm{WF}=2.9 \mathrm{eV}$ ) and Highly Oriented Pyrolitic Graphite (HOPG) biased at $-130 \mathrm{~V}$ inside a $1 \mathrm{~Pa} \mathrm{H}_{2}$ plasma are compared on Fig. 14a. NIEDFs have proven to contain a lot of information on negative-ion production mechanisms [83-84-86]. Negative-ions can be formed through sputtering of an adsorbed hydrogen (or deuterium) atoms [88-89] or through backscattering of an incoming positive ion [88-90]. In both situations the outgoing particle, $\mathrm{H}$ or $\mathrm{D}$, captures an electron on the surface or close to the surface giving a $\mathrm{H}^{-}$or $\mathrm{D}^{-}$negative-ion. One can see on Fig. 14a that on any material most of the ions are created with an initial kinetic energy. This kinetic energy is coming from the sputtering mechanism usually giving a peak of particles at few
$\mathrm{eV}$ and a tail extending slightly [90], or from the backscattering mechanism usually giving a quite flat distribution in energy from 0 to the maximum energy which is equal more or less to the incoming positive ion energy [90]. It has been shown that for carbon material the peak at low energy is well pronounced due to a high proportion of negativeions created through the sputtering mechanism [83, 86]. On the contrary the measured NIEDF is much flatter when surface production occurs mainly through the backscattering mechanism, as it is the case for instance with Gadolinium material [86]. Exact determination of sputtering to backscattering contributions was possible for carbon materials $[83,86]$ but is usually not accessible. However, the ratio between NIEDF peak and NIEDF tail contribution is still a qualitative marker of backscattering versus sputtering contributions. For instance, NIEDF measured with electride material sits between the ones measured with carbon and gadolinium (see Fig. 14a, $\alpha=0^{\circ}$ ). It shows that sputtering is taking part to negative-ion surface production on electride material but probably to a much less extent than on carbon materials and to a higher extent than on gadolinium.

It has been shown previously that only a small part of negative ions is collected since most of them miss the mass spectrometer orifice [84]. When the sample surface is perpendicular to the mass spectrometer axis, negative ions emitted from the surface at low energy and with an angle close to the mass spectrometer axis are preferentially collected. In order to retrieve information on negative-ions emitted at any energy and angle, it is required to perform measurements at different inclination of the sample surface with respect to the mass spectrometer axis. Such measurements are represented on Fig. 14a. By rotating the sample, ions collected initially are no more measured anymore (it is evidenced on Fig. 14a, low energy ions are no more collected) and we collect ions emitted at other angles that were not collected initially (it cannot be evidenced by the graph but can be demonstrated by calculating negative ion trajectories from the sample to the mass spectrometer). Having collected some negative ions emitted at any angle and any energy, it is possible to reconstruct the as-emitted negative ion energy and angle distribution function (NIEADF) [86] which is represented on Fig. 14b for the electride material (it can be compared to the ones obtained with Gd and HOPG by referring to [86]). It can be seen that there is a maximum in negative ions creation at energy of about $35 \mathrm{eV}$ and with angle around $50^{\circ}$. Such high most probable energy for negative ions confirms that production is 
dominated by the backscattering mechanism. Indeed, under the present experimental conditions, from Eq. (3), $\mathrm{H}_{3}{ }^{+}$or $\mathrm{D}_{3}{ }^{+}$fragments can be backscattered with energy up to $45 \mathrm{eV}(135 \mathrm{eV} / 3)$ creating negative-ions with high initial kinetic energy. The difference with the NIEADF obtained for graphite, for which the peak is observed at very low energy, is striking [86]. Nevertheless, some ions emitted at low energy and angle (around $30^{\circ}$ ) can still be seen on the NIEADF and are probably a signature of a small sputtering contribution on the electride material. In the present experiment high negative bias is applied to the sample to favor negative-ion self-extraction from the sample to the mass spectrometer. However, negative-ion sources for fusion operate with no or very low applied bias, a situation in which the positive ion energy is low and below usual sputtering thresholds. Therefore observing only a small sputtering contribution is not an issue in the view of using the electride material in a negative-ion source.

Integration of NIEADF on angle and energy provides a negative ion total count that can be compared between materials. It turns out that the electride material presents an almost similar production efficiency as graphite, and a much more efficient one than molybdenum. However, absolute negative-ion flux outgoing from the sample cannot be obtained from previous measurements due to the fact that the mass spectrometer transmission function is quite complex to get. Therefore, a dedicated diagnostic has been developed for this purpose and is presented in the next paragraph.

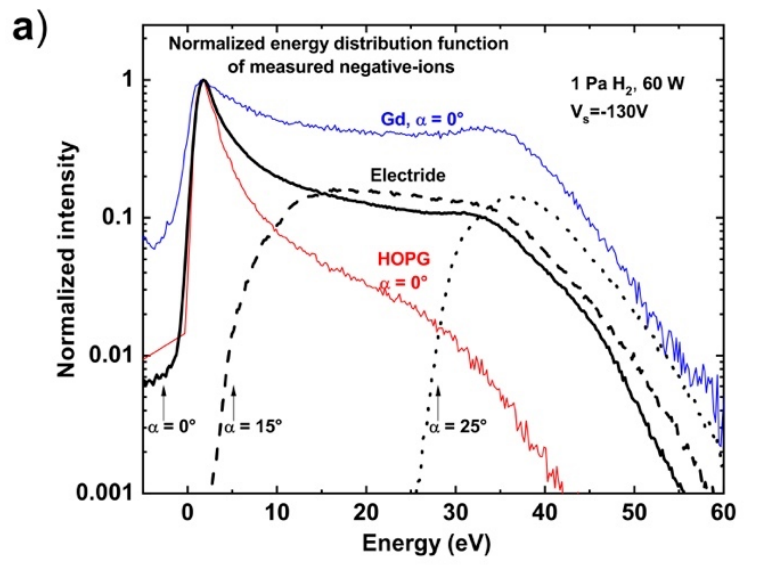

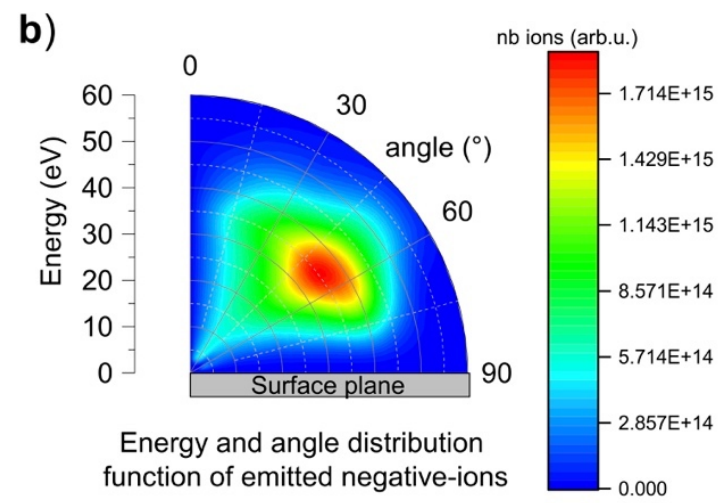

Fig. 14 a) Normalized NIEDF measured in a $1 \mathrm{~Pa}$ hydrogen ECR plasma, $\mathrm{V}_{\mathrm{s}}=-130 \mathrm{~V}$, for different tilt angle $\alpha$ of the sample with respect to the mass spectrometer axis for $\operatorname{Gd}\left(\alpha=0^{\circ}\right)$, HOPG $\left(\alpha=0^{\circ}\right)$, and Electride materials $\left(\alpha=0^{\circ}, \alpha=15^{\circ}, \alpha=25^{\circ}\right)$. b) Polar plot representation of the angle and energy distribution function of negative-ions as emitted by the $\mathrm{C} 12 \mathrm{~A} 7$ electride surface. $\theta=0^{\circ}$ corresponds to the normal to the sample surface plane.

\subsubsection{Magnetized Retarding Field Energy Analyzer measurements}

This diagnostic has first been developed within the context of space propulsion [92]. A detailed description of the Magnetised Retarding Field Energy Analyzer (MRFEA) can be found in the reference [89] and a side-cut view is represented in fig. 15 with its magnetic field lines and dimensions. Briefly, the MRFEA is made of a single-grid RFEA acting as an electrostatic stage and a permanent magnet stacked into a ferromagnetic core acting as a magnetic stage. With a 500 Gauss magnetic field in the barrier, electrons are magnetized and trapped while ions, which are only slightly magnetized, can go through and reach the electrostatic stage. The grounded grid with $30 \%$ transparency associated to the potential-swept copper collector allows selecting ions in energy. Compared to the mass spectrometer, its large entrance opening allows measuring ion current directly rather than working in counting mode, and it is quite straightforward to compute the ion transmission function of the device. Therefore, absolute measurements of negative-ion flux reaching the device are achievable. By calculation of ion trajectories from the sample to the MRFEA it is possible to determine the proportion of emitted negative-ions that reaches the device entrance. This calculation is identical to the one performed for massspectrometer NIEDF analyses (see Sect. 2.3.2.1) and has been validated by several comparisons between experiment and modelling [83,84]. MRFEA measurements combined to this calculation finally provide the absolute negative- 
ion flux emitted by the surface. Unfortunately, measurements have not been performed until now with the C12A7 electride material but with diamond and graphite materials. However, overall counts are close for carbon and electride materials (see Sect. 2.3.2.1).

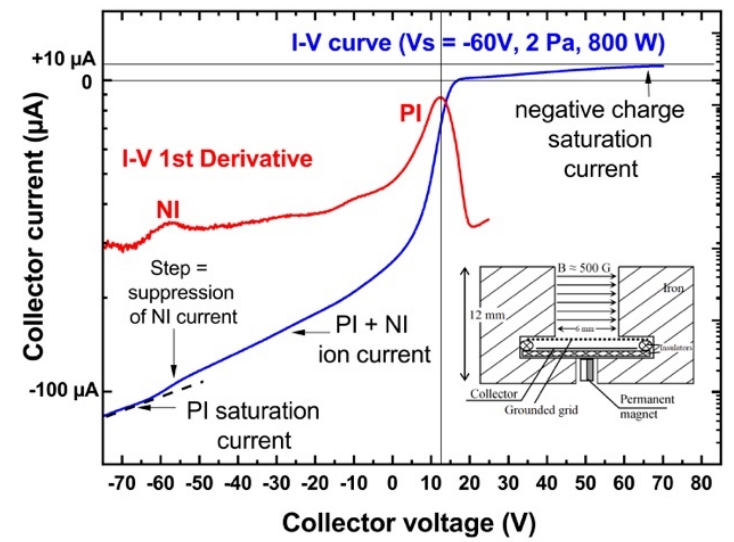

Fig. $15 \mathrm{I}-\mathrm{V}$ curve in blue (and its first derivative in red) measured with the MRFEA in a $2 \mathrm{~Pa}, 800 \mathrm{~W}$ deuterium plasma with sample bias equal to $\mathrm{V}_{\mathrm{s}}=-60 \mathrm{~V}$. The first derivative is giving the positive-ion velocity distribution function with a maximum located at about $15 \mathrm{~V}$ (plasma potential value), as well as the negative-ion (NI) velocity distribution function with a maximum at about $-60 \mathrm{~V}$ (surface bias value). Negative ions measured were coming from the surface of a boron-doped-diamond sample facing the MRFEA for this measurement. Inset: MRFEA cross sectional scheme.

An example of an I-V curve measured with the MRFEA in a $\mathrm{D}_{2}$ plasma, ICP mode, $800 \mathrm{~W}, 2 \mathrm{~Pa}$, using a boron-doped-diamond sample at a bias of $\mathrm{V}_{\mathrm{s}}=-60 \mathrm{~V}$ is depicted on Fig. 15. I is the current collected by the collector while $\mathrm{V}$ is its bias voltage. One can see that the I-V curve presents an unusual shape with a much higher ion current than electron current due to the strong suppression of the electrons in the magnetic barrier. The negative charge saturation current obtained at positive voltage above the plasma potential of about $15 \mathrm{~V}$ here is due to negative-ions coming from the sample but also to electrons escaping the barrier and for that reason cannot be used to determine the negativeion flux. The ion saturation current at negative voltages is due to both positive and negative-ions since the minimum incoming negative- ion energy $\mathrm{E}=-\mathrm{eV}_{\mathrm{s}}(60 \mathrm{eV}$ here $)$ allows them to overpass the electrostatic barrier between the grounded grid and the biased collector. At around $-60 \mathrm{~V}$ negative-ions cannot overcome this electrostatic barrier anymore and the current is due to positive ions only. The step between the positive-ion saturation region at large negative voltages and the ion saturation region (at lower negative voltages) is a measurement of the negative-ion current reaching the collector. Practically, the I-V curve is differentiated to get the negative-ion distribution function (NIEDF, see Fig. 2) and then the NIEDF is re-integrated to get the total negative-current. The proportion of negative-ions reaching the collector among all negative-ions emitted is calculated by taking into account negative-ion trajectories from the sample. The positive-ion flux is measured by Langmuir probes and the negativeion yield (negative to positive ion flux ratio) is estimated from these calculations and measurements.

A negative-ion yield around 3\% has been estimated for pressure between $0.7 \mathrm{~Pa}$ and $2 \mathrm{~Pa}$, power between 200 and $800 \mathrm{~W}$, both for graphite and diamond materials. Measured negative-ion currents range from 100 to $1000 \mathrm{nA}$. These first measurements are of interest since they give the order of magnitude of the negative-ion yield on carbon materials and by extension on the $\mathrm{C} 12 \mathrm{~A} 7$ electride material. For comparison, the negativeion yield obtained with a Cs covered molybdenum converter can reach up to $30 \%$ while negative-ion yield obtained with usual metals like tungsten are usually well below the percent [94]. Several sources of uncertainties are remaining in the yield determination and further works are required before giving more accurate yields. Uncertainties come from the determination of the positive-ion current, MRFEA and sample alignment and finally possible contribution to the measured current from secondary electrons emitted by the sample and escaping the magnetic barrier.

\subsubsection{Photo-Emission Yield Spectroscopy measurements}

The plasma grid work-function in negative-ion sources for fusion can be maintained over quite long periods at a very low value (around $2.1 \mathrm{eV}$ [95]) using continuous injection of caesium. In the case of solid materials such as the $\mathrm{C} 12 \mathrm{~A} 7$ electride, one has to consider that the interaction of the plasma with the material may affect the workfunction. As a consequence, it is of primary importance within the context of negative-ion surface production studies to monitor in-situ the work-function. In that aim, Photo-Emission Yield Spectroscopy (PYS) has been developed and implemented on an ICP plasma chamber.

The PYS setup consists in a $300 \mathrm{~W}$ Xenon lamp combined with a Zolix monochromator to generate a monochromatic light. The photon beam is focused inside the plasma chamber using a lensing system to form a 3-mm diameter spot on the grounded sample. The light wavelength is scanned from 200 to $600 \mathrm{~nm}(6$ to $2 \mathrm{eV})$, and for each 
wavelength the sample current is acquired by a Keithley 616 electrometer and sent to a computer through an amplifier giving the so-called PYS curve. From the PYS curve, the photo-emission threshold which is also the work function is determined. Without light, the sample leakage current is about $30 \mathrm{fA}$ allowing measuring photocurrents in the sub pA range. No measurement can be done in real time in plasma due to the strong sample current, and thus, before measurements the chamber is evacuated to low-pressures, in the range of $10^{-6}$ mbar.

After proper sample preparation, consisting in a baking cycle at $800^{\circ} \mathrm{C}$, and overnight cooling to room temperature in a separate Ultra-Violet Photoelectron Spectroscopy (UPS) chamber (pressure lower than $10^{-9} \mathrm{mbar}$ ), the Electride work-function has been measured equal to $2.4 \mathrm{eV}$ as expected. Let us note that measurement at high temperature and during cooling with a still hot surface gave a higher work function of about 3.4 $\mathrm{eV}$. The same baking cycle has been renewed in the plasma chamber and the work-function has been found similar for the still hot surface $\left(\mathrm{T}_{\mathrm{s}}=500^{\circ} \mathrm{C}\right.$, $\mathrm{WF}=3.4 \mathrm{eV}$, see fig. 16a). However, contrary to the UPS measurement, the work-function has increased to $3.8 \mathrm{eV}$ after one overnight under few $10^{-8}$ mbar (see fig. 16a). Similar effects have been observed in [96] where the work function increased on the minute time scale during sample cooling and reaches $3.9 \mathrm{eV}$ once the sample has cooled down to room temperature. The faster time degradation of the work function in their study is probably a consequence of a two orders of magnitude higher base pressure $\left(10^{-6}\right.$ versus $\left.10^{-8} \mathrm{mbar}\right)$. In the same way, in the present study the difference between WF values after the same baking cycle performed in two different setups probably comes from the higher base pressure in the plasma chamber $\left(10^{-8}\right.$ mbar versus $10^{-10}$ mbar in the UPS setup). It is suspected that residual water vapor in the plasma chamber reacts with the Electride top surface [82] resulting in WF degradation. As a consequence, a study was undertaken to monitor WF after different plasma steps aiming at removing or cleaning the Electride top surface.
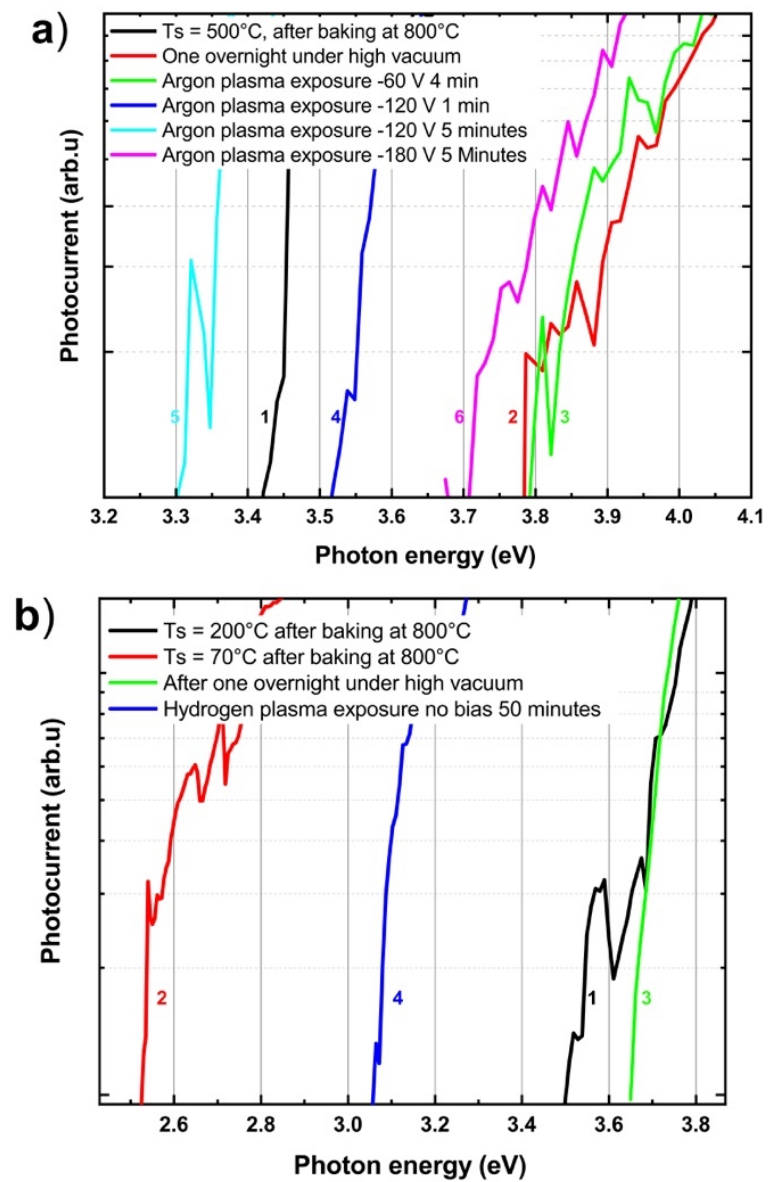

Fig. 16 PYS curves near threshold measured after a) baking at $800^{\circ} \mathrm{C}$ with $\mathrm{T}_{\mathrm{s}}=500^{\circ} \mathrm{C}$ (PYS curve 1 ), one night under high vacuum (PYS curve 2), different exposure to Argon ICP plasmas (0.2, Pa $400 \mathrm{~W}$, PYS curve 3-6), b) baking at $800^{\circ} \mathrm{C}$ with $\mathrm{T}_{\mathrm{s}}=200^{\circ} \mathrm{C}$ (PYS curve 1), baking at $800^{\circ} \mathrm{C}$ with $\mathrm{T}_{\mathrm{s}}=70^{\circ} \mathrm{C}$ (PYS curve 2), one night under high vacuum (PYS curve 3 ), exposure to hydrogen plasma (2 Pa, $200 \mathrm{~W}$, PYS curve 4). The labels correspond to the chronological order of the experiments.

Argon ICP plasmas at $0.2 \mathrm{~Pa}$ and $400 \mathrm{~W}$ were initiated with sample biased at $-60 \mathrm{~V},-120 \mathrm{~V}$ or $-180 \mathrm{~V}$ with the aim of removing by sputtering the modified top layer. After each plasma exposure, the sample was left cooling down to room temperature and the WF was measured. Fig. 16a depicts PYS curves after the different plasma exposures with an indication of the experimental chronology. The PYS curve labeled 1 with $\mathrm{WF}=3.4$ $\mathrm{eV}$ corresponds to the result obtained after the baking cycle with a still hot electride surface. The PYS curve measured after one night under vacuum is denoted as 2, and the WF is around $3.8 \mathrm{eV}$. First, the sample was biased to $-60 \mathrm{~V}$ for 4 minutes and the WF did not change (PYS curve 3 ). We conclude that $60 \mathrm{eV} \mathrm{Ar}$ ions are not energetic enough to sputter the top layer. Then, $-120 \mathrm{~V}$ was applied for 1 minute and the WF decreased down to $3.5 \mathrm{eV}$ (PYS curve 4), which is still higher than the initial 
WF value of $3.4 \mathrm{eV}$. Thus, only a fraction of the top insulating layer is expected to have been removed or cleaned after 1 minute. Further exposure for 5 minutes decreased the WF down to $3.3 \mathrm{eV}$, close to its initial value of $3.4 \mathrm{eV}$. Finally, $-180 \mathrm{~V}$ was applied for 5 minutes and the WF increased above $3.6 \mathrm{eV}$, suggesting that $180 \mathrm{eV}$ Argon sputtering damages the surface. Ar cleaning at $-120 \mathrm{~V}$ can thus be used as a cleaning procedure for electride after vacuum storage. Note that we witnessed no WF change greater than $0.1 \mathrm{eV}$ after 2 hours exposure to vacuum. Thus, $\mathrm{H}_{2} \mathrm{O}$ molecules are not expected to influence the WF between two measurements.

Besides electride WF evolution under Ar plasmas, the influence of $\mathrm{H}_{2}$ plasma exposure was studied. First, the sample was baked up to $800^{\circ} \mathrm{C}$. The WF measured during cooling with a still hot surface $\left(\mathrm{T}_{\mathrm{s}}=200^{\circ} \mathrm{C}\right.$ on figure $\left.16 \mathrm{~b}\right)$ is $3.5 \mathrm{eV}$, close to the previous measurement (Fig. 16a curve 1) but this time the WF was also measured with the sample at low temperature $\left(\mathrm{T}_{\mathrm{s}}=70^{\circ} \mathrm{C}\right.$ on Fig. 16b) before storage under high vacuum. A WF of $2.5 \mathrm{eV}$ was found, quite close to the expected $2.4 \mathrm{eV}$ value. However, after one night the WF re-increased to $3.6 \mathrm{eV}$ (Fig. 16b) as already observed on Fig. 16a. Then, an ICP $\mathrm{H}_{2}$ plasma ( $2 \mathrm{~Pa}, 200 \mathrm{~W}$ ) was initiated with the sample left floating (no bias case). We can see on Fig. 16b that the electride WF is decreased down to $3.05 \mathrm{eV}$ going even below the minimum WF value obtained after argon plasma $(3.3 \mathrm{eV})$. Therefore, $\mathrm{H}_{2}$ plasma at low positive-ion impact (no bias case) seems to help reconstructing or cleaning the Electride surface, allowing to retrieve a low WF. The beneficial effect of hydrogen plasma exposure on the electride work-function has also been observed in [96]. In this work, a work function of $2.75 \mathrm{eV}$ has been reached after 2 hours of hydrogen ICP plasma exposure (10 Pa, $250 \mathrm{~W})$. The lower work function value obtained in their work might come from the longer and higher density plasma used.

Several exposures to hydrogen plasma with a -60 $\mathrm{V}$ bias tended to show that the WF re-increases compared to the no bias case. Hydrogen penetration inside the material at higher positiveion impact seems to be detrimental to the WF. This effect has also been observed in [97] where more systematic measurements were performed with an applied bias. Let us note that negative-ion sources for fusion usually operate at low positive-ion energy with a plasma grid biased slightly below the plasma potential [98]. As a consequence, these preliminary results on $\mathrm{C} 12 \mathrm{~A} 7$ electride material exposed to hydrogen plasmas can be considered as encouraging.

\section{Innovative concepts for negative ion production in Cs-free plasmas}

As mentioned in the introduction section, no Csfree ion source fulfills ITER requirements for coextracted electron and duration. Nevertheless, ECR $(2.45 \mathrm{GHz})$ plasma sources already employed (section 2.2) as a solution to mimic plasma conditions observed in full-scale ion source or in edge-plasma could be employed in a network for fundamental studies of the production of negative ion at larger scale. The reactor PROMETHEUS I in operation at Patras University (Greece) with five plasma sources is a valuable device for such refine studies. At RFX-INFN (Padova, Italy), the NIO1 negative ion source and its ancillaries are devoted to evaluate all the NBI components from the Csoven to the energy recovery device to improve the NBI efficiency or even less conventional studies on gas feeding $\left(\mathrm{O}_{2}\right)$ are carried-on. Last part of this section describes recent results obtained on the RAID reactor where a helicon antenna sustains a $1.5 \mathrm{~m}$ long Cs-free plasma column where laserphotodetachment and CRDS measurements probed $\mathrm{H}_{2}$ and $\mathrm{D}_{2}$ plasma of $2 \times 10^{16} \mathrm{~m}^{-3}$ of negative ions at $5 \mathrm{~kW}$ and $0.3 \mathrm{~Pa}$.

\subsection{PROMETHEUS reactor at Patras University}

The negative ion source "Prometheus I" [64,99] lies in the field of the Electron Cyclotron Resonance (ECR) driven negative ion sources [100]. It consists of a cubic ( $24 \mathrm{~cm}$ inner edge) stainless steel chamber with different viewports for diagnostic tool installation. The base pressure is about $3 \times 10^{-6}$ Torr (Fig. 17a). The plasma is sustained by a two-dimension network of five ECR-plasma elementary sources. An ECR module consists of a microwave guide terminated at a permanent magnet. Each module is driven by an independent microwave solid state power supply $(2.45 \mathrm{GHz})$ able to provide up to $180 \mathrm{~W}$. Pure $\mathrm{H}_{2}$ $(99.999 \%)$ or $\mathrm{D}_{2}(99.8 \%)$ gas is introduced by means of digital mass flow controller at flow rates 3.9-23.4 sccm and 4.8-29.2 sccm, respectively. The working (plasma on) gas pressure varies, respectively, between 2 and 20 mTorr in both cases. 
(a)
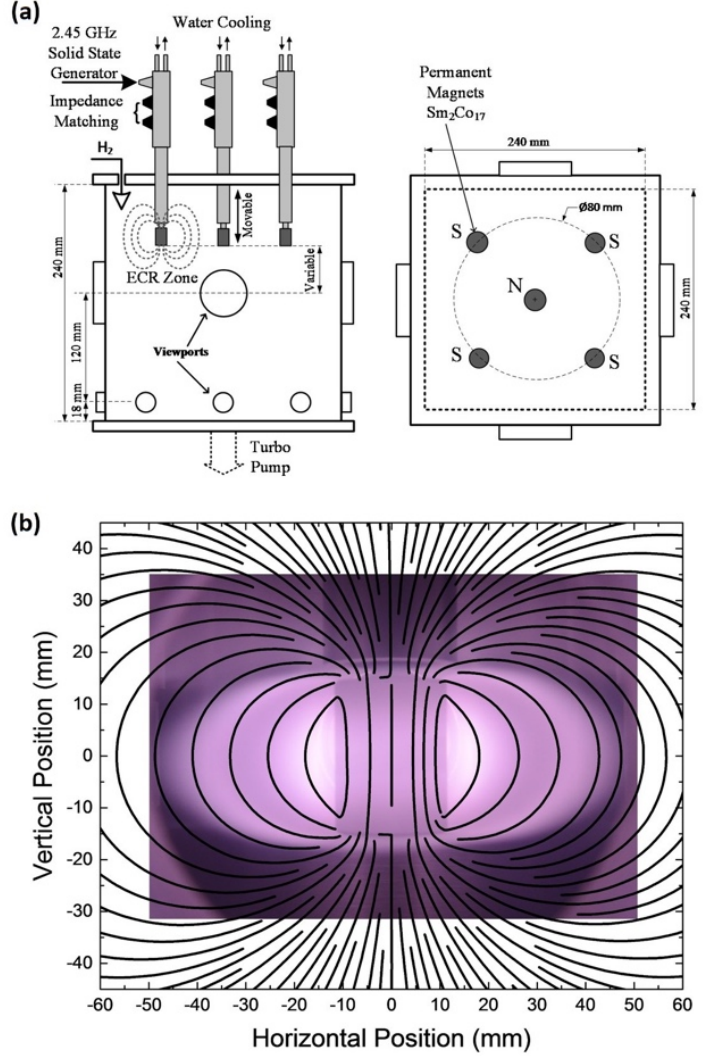

Fig. 17 a) Conceptual design of the ECR-driven negative ion H-/D- source "Prometheus I" (side and top views). b) Streamlines of the magnetic field of an elementary ECR source superimposed on a representative photograph of the corresponding visible plasma ( $\mathrm{H}_{2}$ case here).

The magnetic field of a single ECR module is simulated using a commercial numerical suite (COMSOL Multiphysics) [64] and the result is depicted in Fig. 17b. The magnetic field streamlines are compared in the same figure with the visible plasma generated in an ECR zone (conventional photography). The high-energy electrons which gain their energy in the ECR zones appear to be mostly confined within the luminous region, in the vicinity of the permanent magnet, where they promote gas excitation and dissociation. Hence, with this configuration, the ECR magnetic field also plays the role of the magnetic filter, allowing mainly low-energy electrons and excited neutrals to diffuse into the bulk plasma, i.e. downstream of the ECR zones.

The produced plasma is studied by means of electrostatic probe and laser photo-detachment. The probe is made of a $0.25 \mathrm{~mm}$ in diameter tungsten wire and the tip (exposed to the plasma) is L-bent to be aligned with the laser beam for the photo-detachment measurements. The tip is $15 \mathrm{~mm}$ in total length with the bent part being $11 \mathrm{~mm}$. The rest of the wire is housed in a telescopic configuration of dielectrics (alumina tube inside a wider quartz tube) that insulates and protects it from the plasma. The quartz tube is supported inside a stainless-steel tube that ends in a standard bayonet Neill-Concelman vacuum feedthrough. A conflat flange-to-quick connect coupling adapter makes a vacuum joint with the steel tube and at the same time allows the linear translation of the probe. The acquisition of the electrostatic probe currentvoltage curves is accomplished with a custommade standalone system [101]. Each measurement procedure includes $10 \mathrm{~s}$ of probe cleaning by electron current induced incandescence followed by another $10 \mathrm{~s}$ of cooling-down. The acquisition is realized point by point in steps of $97.6563 \mathrm{mV}$. For each point, $2^{12}$ current-voltage samples are averaged in order to reduce the plasma induced noise.

For the present experiments, the probe is positioned at the center of the source and $65 \mathrm{~mm}$ lower than the middle plane of the central ECR module. The magnetic field of an ECR module has vertical downward direction (Fig. 17b) and a magnitude of 51 Gauss, while the probe tip itself is oriented horizontally (i.e. perpendicular to the magnetic field). The lowest electron temperature observed during the present experiments is about $0.5 \mathrm{eV}$ which corresponds to an electron Larmor radius of about $0.34 \mathrm{~mm}$. Thus, even in the worst case, the probe radius $(0.125 \mathrm{~mm})$ is sufficiently smaller than the electron Larmor radius, validating the use of the conventional non-magnetized probe theory.

The photo-detachment diagnostic technique with one laser beam is employed for the determination of the negative ion $\left(\mathrm{H}^{-}\right.$and $\left.\mathrm{D}^{-}\right)$ absolute density. A short ( $\sim 5 \mathrm{~ns}) \mathrm{Nd}$ :YAG $1064 \mathrm{~nm}$ laser pulse, generated from a Quantel Brilliant EaZy unit, is concentrically aligned with the bent part of the probe tip and detaches the extra electron of negative ions inside the irradiated cylindrical volume. The excess (i.e. photo-detached) electrons, in the section of the irradiated volume that contains the bent part of the probe tip, sharply increase the electron current collected by the positively biased probe. The density of the negative ions can then be calculated from the amplitude of that current pulse. To avoid any potential errors that arise from the use of the traditional capacitive decoupling circuit, a wideband current transformer (Pearson electronics $6585 ; 400 \mathrm{~Hz}-200 \mathrm{MHz}$ ), connected directly to a digital oscilloscope (LeCroyWaveSurfer 104Xs-A; $1 \mathrm{GHz} ; 5$ GSample $\mathrm{s}^{-1}$ ), is used to measure the above current pulse. Various parameters of the photo-detachment technique are properly set for its valid application [102]. Firstly, the laser radius is chosen to be $2.5 \mathrm{~mm}$ which sufficiently exceeds the 
probe collection radius $(\sim 0.1 \mathrm{~mm}$ which is the typical Debye length for the present experimental conditions). Secondly, the probe bias is set to +15 $\mathrm{V}$ (i.e. 7-8 $\mathrm{V}$ above plasma potential) which is sufficient for collecting all the detached electrons without causing the incandescence of the probe tip due to electron current. To establish this, a series of measurements was realized which demonstrated that a $+15 \mathrm{~V}$ bias leads to the saturation of the photo-detached electron current. Lastly, the energy density of the laser beam is chosen to be around 70

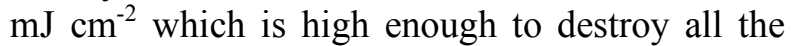
negative ions in the irradiated volume. This is ensured by reaching the saturation of the photodetachment signal versus the laser power.

In the source "Prometheus I" negative ions originate in the bulk plasma, where the volume production mechanism takes place. Contrary to surface production sources, volume production sources operate with pure $\mathrm{H}_{2}$ or $\mathrm{D}_{2}$, having the practical advantage of inherently caesium-free operation, which makes them an attractive alternative. While different processes might be responsible for the formation of negative ions $[64,70,71,99]$, the volume production mechanism is an efficient path and mainly refers to the process of dissociative attachment (DA) of low-energy (cold) electrons to highly vibrationally excited molecules $[18,64,70,99]$ :

$\mathrm{H}_{2}\left(X^{1} \Sigma_{g}^{+} ; v\right)+e_{\text {cold }} \rightarrow H_{2}^{-} \rightarrow H^{-}+H$

$D_{2}\left(X^{1} \Sigma_{g}^{+} ; v\right)+e_{\text {cold }} \rightarrow D_{2}^{-} \rightarrow D^{-}+D$

It is important to note that there is a substantial difference in the cross sections for DA between $\mathrm{H}_{2}$ and $\mathrm{D}_{2}$. Indicatively, near the thresholds of two extreme levels, i.e. $v=0$ and $v=9$, the following stand in $\mathrm{H}_{2}$ vs. $\mathrm{D}_{2}$, respectively: $1.6 \times 10^{-5} \AA^{2}(3.73$ eV) vs. $3.83 \times 10^{-8} \AA^{2}(3.83 \mathrm{eV})$ and $4.8 \AA^{2}(0.13 \mathrm{eV})$ vs. $2.3 \AA^{2}(1 \mathrm{eV})$ [16]. It is seen that, for the lower vibrational levels this difference is around three orders of magnitude but drops to less than one order of magnitude as the vibrational level increases. At the same time, the near threshold cold electron temperature tends to be slightly higher in the $\mathrm{D}_{2}$ case.

As regards the molecular precursors of the above reactions, an effective way for highly vibrationally excited molecule formation is related to ground state molecule excitation by energetic (hot) electrons to singlet electronic states, followed by spontaneous emission $[18,55,64,99]$ :

$$
\begin{gathered}
H_{2}\left(X^{1} \Sigma_{g}^{+} ; v_{i}=0\right)+e_{\text {hot }} \rightarrow \\
H_{2}\left(B^{1} \Sigma_{u}^{+}, C^{1} \Pi_{u}^{+}, \ldots\right)+e_{\text {cold }} \rightarrow H_{2}\left(X^{1} \Sigma_{g}^{+} ; v_{f}\right)+ \\
e_{\text {cold }}+h v
\end{gathered}
$$

$$
\begin{aligned}
& D_{2}\left(X^{1} \Sigma_{g}^{+} ; v_{i}=0\right)+e_{\text {hot }} \rightarrow \\
& D_{2}\left(B^{1} \Sigma_{u}^{+}, C^{1} \Pi_{u}^{+}, \ldots\right)+e_{\text {cold }} \rightarrow D_{2}\left(X^{1} \Sigma_{g}^{+} ; v_{f}\right)+ \\
& e_{\text {cold }}+h v
\end{aligned}
$$

Available cross section data [18] reveal that this process is isotope dependent for lower values of the final vibrational level, $v_{f}$, whereas for high lying levels $\left(v_{f} \geq 5\right)$, which are the ones that predominantly participate in reactions (4) and (5) towards the formation of negative ions [103], the cross sections converge. In both cases this reaction is favorable for hot electron temperatures greater than $10 \mathrm{eV}$ [104]. Depending on the operational parameters and the design of the ion source, an additional important way leading to vibrational states is the recombinative desorption of atoms on the surface of various materials $[48,55]$. Thus, gas retention in the source walls may be crucial for the source efficiency.

As mentioned above, the source "Prometheus I" operates either with $\mathrm{H}_{2}$ or $\mathrm{D}_{2}$ gas. Hence, special attention is paid to eliminate as much as possible the gas retention when switching from one gas to the other. Thus, the source walls are uniformly heated at $150^{\circ} \mathrm{C}$ during a few successive working days (typically $3 \times 10 \mathrm{~h}$ ) at base pressure conditions. Since this effect hasn't yet been studied quantitatively, the above protocol is adopted following numerous experiments in order to achieve repeatability and reproducibility of the plasma parameters between gas interchange. On the contrary, it is established that for meeting the above prerequisites by wall cleaning merely by means of continuous plasma operation, a monthscale period is required. Moreover, during the experimental procedure, since the wall temperature is not controlled, the final temperature of the surfaces is mainly determined by the operating parameters, i.e. the inserted power and the gas pressure. Therefore, prior to each individual measurement and after setting both parameters, the source is thermally stabilized for over approximately $20 \mathrm{~min}$.

Overall results demonstrate the production of two different electron populations (i.e. cold and hot), independently of the operating conditions, both in the $\mathrm{H}_{2}$ and $\mathrm{D}_{2}$. The hot electrons gain energies between about $12.5 \mathrm{eV}$ and $17 \mathrm{eV}$ without significant deviations between the two gases, independently of the operating parameters. Similarly, their densities do not deviate in the two gases versus the pressure and the power, and get values up to $8 \times 10^{8} \mathrm{~cm}^{-3}$. The cold electrons gain energies between about 0.5 and $2.0 \mathrm{eV}$, but according to Fig. 18a and the inset therein their density and temperature tend to be higher in $\mathrm{D}_{2}$. 

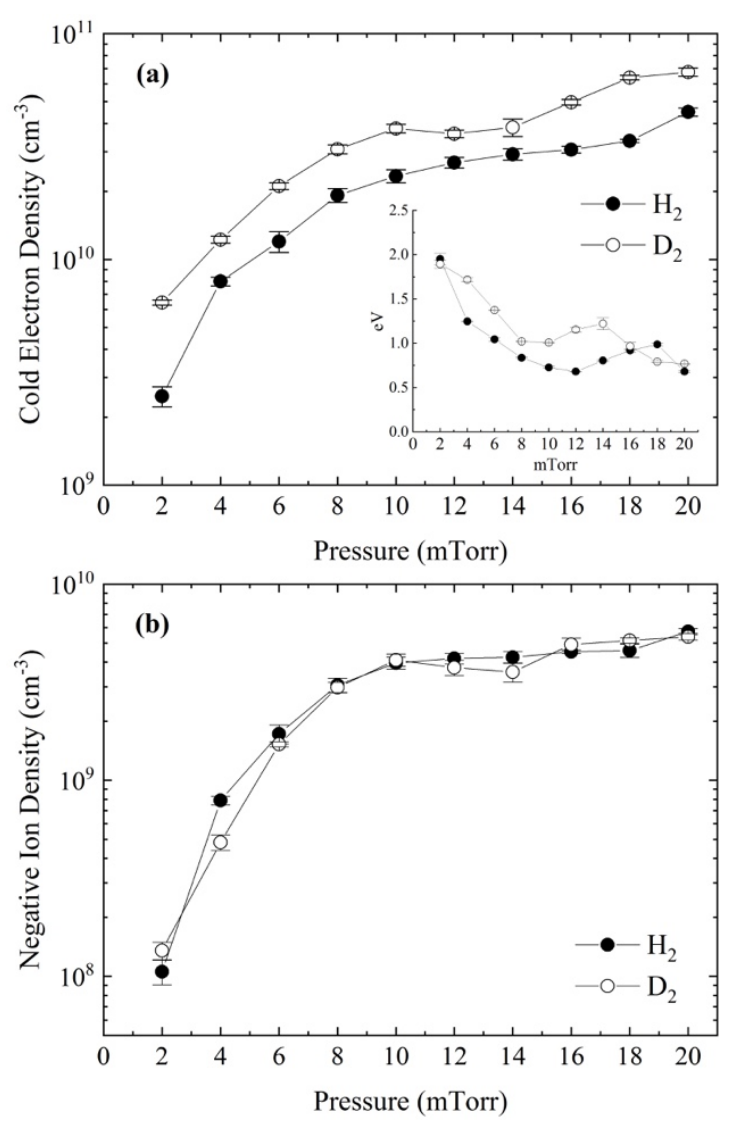

Fig. 18 Representative results from the source "Prometheus I" operating either with $\mathrm{H}_{2}$ or $\mathrm{D}_{2}$ gas. The inserted microwave power is $0.9 \mathrm{~kW}$, equally distributed by the five ECR modules. a) Cold electron density vs. pressure; (inset) Cold electron temperature vs. pressure; b) Negative ion $\left(\mathrm{H}^{-}\right.$and $\left.\mathrm{D}^{-}\right)$density vs. pressure. Mean values and error bars are calculated from four measurements of two independent experiments.

These results are consistent with the volume production concept. The hot electrons promote $\mathrm{EV}$ excitation and the cold ones yield negative ions through DA. Fig. 18b presents a direct comparison between the absolute densities of the $\mathrm{H}^{-}$and $\mathrm{D}^{-}$ions formed in the source, across the entire operating pressure range. The density monotonously increases up to about 10 mTorr, in both gases. Quasi-saturation around the value of about $6 \times 10^{9}$ $\mathrm{cm}^{-3}$ (20 mTorr) is finally reached.

The diminishing trend of the density of the cold electrons and the negative ions with increasing pressure (Fig. 18) has been previously observed experimentally and predicted numerically [103] in different source configurations. As regards the main species considered here, i.e. $\mathrm{H}^{-}$and $\mathrm{D}^{-}$ions, two physical effects should be taken into account for a qualitative interpretation of this tendency: (i) the limited formation of highly ro-vibrationally excited molecules at higher pressures [103] and (ii) the dependence of main negative ion destruction processes on the gas pressure $[64,103]$. As regards (ii), associative detachment (AD) of negative ions with neutral atoms, mutual neutralization $(\mathrm{MN})$ with positive ionic species, and electron detachment (ED) upon collisions with electrons are the most significant ones [64, 99, 70]. These processes appear to be enhanced as the $\mathrm{H}_{2}$ pressure increases in a range similar to the present experimental conditions [64, 103].

Then, it is interesting that the absolute negative ion yield is nearly the same in both discharges (Fig. 18b). The present data are not adequate to fully discuss this observation, but the following remarks consider some relevant principles:

It is speculated that within the range of interest (5 $\leq v \leq 11)$ the vibrational distribution functions (VDFs) for $\mathrm{H}_{2}$ and $\mathrm{D}_{2}$ are alike, since the cross sections for the EV process converge with increasing vibrational level [18] and the measured energies of the hot electrons are similar in both gases. In the case of $\mathrm{D}_{2}$, a larger negative ion yield would be anticipated owing to the higher cold electron density (Fig. 18a). But this may be compensated by the smaller value of the DA cross section (reaction (5)), accountable thus for the close values of measured $\mathrm{H}^{-}$and $\mathrm{D}^{-}$densities. It is also noted that the DA reaction is equally efficient in both gases with respect to the electron energy near threshold values (see inset of Fig. 18a and discussion following reactions (4) and (5)). Finally, as it was implied above, it is the balance between formation and destruction processes that ultimately dictates the steady state negative ion density. Hence, it is also important to consider the contribution of the destruction processes in this analysis. It is just mentioned here that, in "Prometheus I" AD has been identified as the dominant process of $\mathrm{H}^{-}$destruction, while it has been reported [105] that there is no significant isotope effect in the total cross section for AD.

\subsection{NIO1 at RFX-INFN}

The production of $\mathrm{H}^{-}$ions in a plasma source requires carefully balanced plasma conditions [71]: in first instance, two plasma regions are necessary, one region (named driver) with electron temperature $\mathrm{T}_{\mathrm{e}}>4 \mathrm{eV}$ to efficiently excite or dissociate $\mathrm{H}_{2}$ molecules, the second (near extraction) with $\mathrm{T}_{\mathrm{e}}<2 \mathrm{eV}$ to allow accumulation of $\mathrm{H}^{-}$ion (binding energy $0.76 \mathrm{eV}$ ); charge exchange on caesiated surfaces is more effective $[71,106,107]$ than volume production by DA, but adds the complexity of surface conditions. Beam extraction, voltage holding and beam neutralization are also dependent from source condition [109-112]. 
$\mathrm{H}^{-}$sources are thus a good example of a complex system; to list a few key issues:

(a) magnetic filter must protect extraction from hot thermal electrons, without inducing excessive plasma drifts and non-uniformity or excessively depressing the local plasma density [113];

(b) the plasma energizing by RF heating improve durability (as compared to arc), but produces hot thermal electrons with typical $\mathrm{T}_{\mathrm{e}}$ difficult to control ( $\mathrm{T}_{\mathrm{e}}$ from 4 to $10 \mathrm{eV}$ ) $[107,114,115]$;

(c) the coextracted electron current perturbs the meniscus from which $\mathrm{H}^{-}$are extracted [116].

A relatively compact $\mathrm{H}^{-}$sources, featuring 9 extraction holes (in $3 \times 3$ matrix) and $98 \mathrm{~mm}$ diameter plasma chamber, named NIO1 (Negative Ion Optimization 1), was developed in close collaboration between Consorzio RFX and INFNLNL to investigate these physical issues, and to provide experimental experience on source adjustment and possibly validation of new ideas [117]; in particular NIO1 can operate with several external Cs ovens or without them; filter, gas and plasma chamber liners are also changeable. NIO1 was commissioned at Consorzio RFX since 2014 with air as process gas, while use $\mathrm{H}_{2}$ as process gas was approved in 2015; a Cs oven separately developed in a test stand was finally installed on NIO1 in the 2019 Winter; before this, data on Csfree NIO1 operation were systematically collected. NIO1 set-up was described elsewhere [117-119]; its main section is shown in Fig. 19. Major design goals were: capability of continuous operation regimes (and extended operational databases and experience to recognize complex phenomena in $\mathrm{H}^{-}$ source physics); robustness of radiofrequency tuning and plasma ignition (also called plasma onset); comparison of Cs-free and Cs operation; improvement of filter design and validation of related simulations.

Concerning last Cs-free operation, a source wall conditioning effect by Oxygen plasma was noted [118], and a successful campaign to extend this effect to other gases $\left(\mathrm{N}_{2}, \mathrm{Ar}, \mathrm{Xe}\right)$ is recalled here. The optimal magnetic filter strength proved rather large (12 $\mathrm{mT}$ order), so that a significant nonuniformity among beamlets is often detected by lateral cameras (see later); tomographic reconstruction of extracted beam is also well advanced [120].

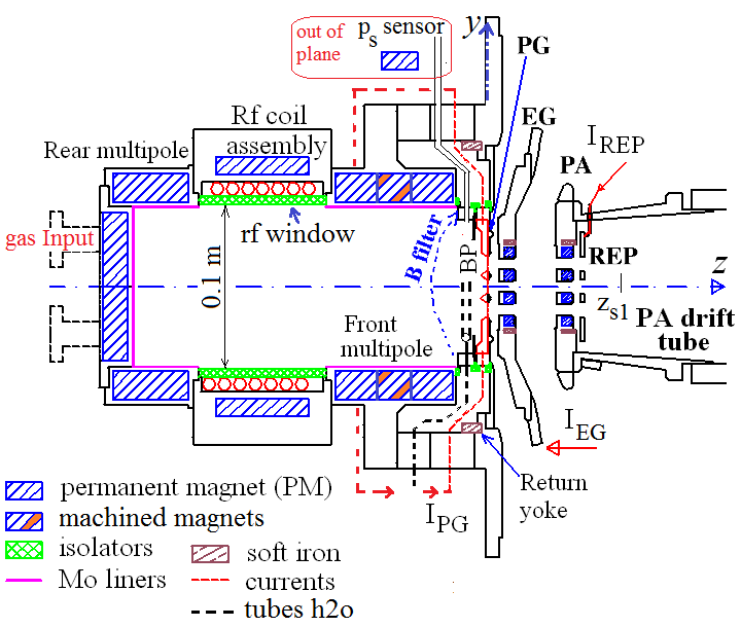

Fig. 19 NIO1 modified setup: yz section, also with some out of plane parts: gas sensor tube; additional filter magnets. Note that some front multipole magnets were shaped, the filter current path $\mathrm{I}_{\mathrm{pg}}$ was modified; bias plate $\mathrm{H}_{2} \mathrm{O}$ cooled tubes shown in part.

In 2019 end a first Cs oven was commissioned; the obtained increment of $\mathrm{I}_{\mathrm{a}}$ by a factor 2 does not yet satisfy expectation of a factor 10 ; but note that, due to present emergency situation, optimization of oven operation temperature is not finished, and some discussion on time evolution of undercaesiation and over-caesiation regimes is reported elsewhere [123]; more careful studies are needed and are being organized, with possibility of further $\mathrm{I}_{\mathrm{a}}$ increment.

Study of new concept for energy recovery (see Fig. 20) were recently adapted to space limitation typical of NIO1 [122] or other similar sources; note that these energy recovery studies are not funded by Eurofusion. Other upgrades of beam diagnostic (including a strongly cooled pepper pot calorimeter and removable faraday cup) and of ion source (Faraday screen, dual gas injection) are foreseen.
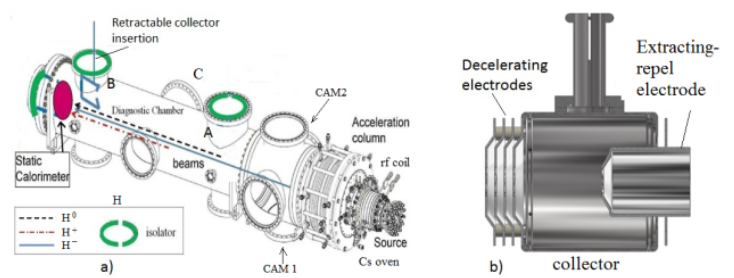

Fig. 20 (a) overall scheme of NIO1 beam diagnostic chamber; note that positions A or B may host the energy recovery plugin or a removable Faraday cup; currently 'A' hosts the CFC tile, while 'B' may host a pump or be a spare; (b) detail of Energy recovery plugin.

\subsubsection{Basics of NIO1 radiofrequency operation}

The turning on of plasma (onset or ignition) is a complex phenomenon, involving a phase of electron multiplication; moreover in a RF source no direct initial ionization by an arc is available. Few general physical remarks are noted in this 
paragraph: two basic kind of coupling are known, capacitive coupled plasma (CCP) and inductive coupled plasma (ICP), which prevails when RF electric penetration is shielded by skin effect (perhaps helped by surface conductivity of RF window or proximity of metallic parts) or by a real Faraday shield. Considering NIO1 case, a plasma density $n_{e}>>3 \times 10^{17} \mathrm{~m}^{-3}$ in the driver is necessary for producing enough ion current; and a typical scale of skin depth is $\delta_{0}=\mathrm{c} / \omega_{\mathrm{pl}}[121]$ where $\omega_{\mathrm{pl}}$ is the angular plasma frequency, which gives $\delta_{0}$ about 9 $\mathrm{mm}$, still well consistent with ICP. In this regime, electron acceleration happen by accumulation of kicks, so a bell shaped energy distribution (similar to but not necessarily a Maxwellian one $[114,115]$ ) should be expected with relatively poor ionization efficiency as compared to an arc; anyway plasma may start in CCP regime and immediately progress to ICP regime, which transition deserves future simulations.

Simplified simulations assuming ICP regime [124] show that the positive feedback of $n_{e}$ on itself have to be stabilized by gas density $n_{\mathrm{g}}$ depletion or other nonlinearities of the system, possibly with multiple stationary equilibrium points (multistable) and of course dependent on additional $n_{e}$ sources (emission from walls) and electron trajectory in RF fields, which request additional experimental effort in controlling trends.

Up to now, the process gas is fed on the source rear, with a $\mathrm{V}_{\mathrm{c}}$ voltage controlled valve; the source gas pressure $p_{s}$ is read with a pipe connected near the front side of the plasma chamber, with software feedback on $V_{c}$ possible; a second gas input on the front is being installed in this winter shutdown. The 7 turn RF coil is insulated by air and a thermoplastic jacket, and resonates with its matching box (MB) at a frequency $f_{s}$ (about 2 $\mathrm{MHz}$ ) which slightly depends on plasma condition (density) and process gas (see later). The solid state $\mathrm{RF}$ generator is operated at an assigned forward RF power $P_{k}$ and driving frequency $f_{r}$. Up to now NIO1 has no Faraday shield, so RF power can be directly coupled to plasma, both capacitive and inductive coupling plasma can be observed, and RF window is directly exposed to plasma. The capacitively coupled plasma (low density, very low current) may turn on at low power, but more effective inductive coupling prevails for $P_{k}>1 \mathrm{~kW}$. NIO1 has no auxiliary systems for plasma ignition (also known as plasma onset, $\mathrm{n}_{\mathrm{e}}$ rising from 0 to an equilibrium point), and mechanical MB tuning is possible, but not used, since a satisfactory plasma onset transition is obtained by a simple $\mathrm{f}_{\mathrm{r}}$ shift and $\mathrm{a} \mathrm{p}_{\mathrm{s}}$ overshoot (controlled by $\mathrm{V}_{\mathrm{c}}$ ). In typical operation with $\mathrm{H}_{2}$ process gas, reflected power below 5\% (and sometimes below 1\% which is the generator detection accuracy) is achieved with $\mathrm{f}_{\mathrm{r}}$ from 2008 to $2014 \mathrm{kHz}$, for a wide range of source pressure $\mathrm{p}_{\mathrm{s}}$ from 0.2 to $1 \mathrm{~Pa}$ and RF power $\mathrm{P}_{\mathrm{k}}$ from 1 to $1.8 \mathrm{~kW}$; from this we can infer that matching box impedance $Z_{b}$ is about $50 \mathrm{ohm}$ [123]. Without plasma, $Z_{b}$ can be directly measured and has a maximum of about $150 \mathrm{ohm}$ with no reactance for $\mathrm{f}_{\mathrm{r}}$ near $2010 \mathrm{kHz}$; to avoid large reflections, we detune $\mathrm{f}_{\mathrm{r}}$ to $2020 \mathrm{kHz}$ until plasma turns on.

By switching process gas to $\mathrm{O}_{2}$ or $\mathrm{N}_{2}$ or $\mathrm{Ar}$ or $\mathrm{Xe}$ [123], it was noted that the $\mathrm{f}_{\mathrm{r}}$ used for minimizing RF reflection changed, ranging from about 2014 $\mathrm{kHz}$ used for oxygen or nitrogen (the difference with $\mathrm{H}_{2}$ case are within tolerance of the reflection optimization) to the $2025 \pm 2 \mathrm{kHz}$ of Ar case to the $2035 \pm 5 \mathrm{kHz}$ of the Xe case. This correlates to element $Z$, indicating that the larger availability of electrons makes coil + plasma inductance lower (and consequently resonating frequency higher) and it offers a valuable test bench for simulation of radiofrequency coupling.

\subsubsection{Energy recovery}

An important part of a Neutral beam injector is the conversion of fast $\mathrm{H}^{-}$ions to fast $\mathrm{H}^{0}$ atoms; some concepts promise high conversion efficiency $\eta$, as photoionization (near $100 \%$ ) or plasma neutralizer (near $80 \%$ ), but consolidated design by gas collision is limited to $\eta<60 \%$ with spurious $\mathrm{H}^{+}$and $\mathrm{H}^{-}$fractions which must be deflected or removed from beam line, both to protect following parts (especially tokamak) and possibly to recover beam energy in a decelerator. The magnetic deflection may require considerable energy and large yokes, so electrostatic deflection is usually preferred (with possible issues of sheath stability). Our concept joins deceleration and ion removal, achieved by the defocusing due beam deceleration itself [122]: so the secondary electron emission (SEE) due to stopped $\mathrm{H}^{-}$impact is minimized (to Eurofusion request, we repeat that this concept is not funded by them, as noted before). To fit inside a CF200 side port (many ports are available in NIO1, see fig $20 \mathrm{a}$, especially $\mathrm{B}$ or $\mathrm{C}$ ) the proposed prototype is limited to $20 \mathrm{kV}$ beams, with typical collector voltage $\mathrm{V}_{\mathrm{c}}=-19.9 \mathrm{kV}$ and repel voltage $\mathrm{V}_{\mathrm{r}}=-20.3$ $\mathrm{kV}$; SEE back streaming is suppressed by low impact energy and radial geometry, as shown by simulations [122].

Note that $\mathrm{H}^{+}$beam recovery is possible with a subsequent similar electrode set, with much easier SEE trapping; moreover, the plugin can be tested also on different sources [122]. Moreover, the NIO1 cooled calorimeter installation is slowly progressing, allowing some isolator addition for 
testing some energy recovery capability; calorimeter also integrates a pepper pot hole array for possible emittance measurement.

\subsubsection{Magnetic filter}

Source filter field $\mathrm{B}^{\mathrm{s}}$ was progressively increased in NIO1, up to $12 \mathrm{mT}$ total, to reduce the large coextracted electron current. In this progression [118], no detrimental effect on RF coupling were detectable. Producing this field by $\mathrm{I}_{\mathrm{pg}}$ current alone $\left(\mathrm{B}_{\mathrm{x}}{ }_{\mathrm{x}}\right.$ in ref [118] notation) is not possible, and two other terms $\left(\mathrm{B}^{3}{ }_{\mathrm{x}}\right.$ and $\left.\mathrm{B}_{\mathrm{x}}{ }_{\mathrm{x}}\right)$ were added, produced by rectangular bars of $\mathrm{SmCo}$; here $\mathrm{I}_{\mathrm{pg}}$ is the current running through the $\mathrm{PG}$ (with an adequate return path), as skectched in Fig 19. The elaborate contribution $\mathrm{B}_{\mathrm{x}}^{3}$ is made by removing some part of the NIO1 front multipole bars (placed in vacuum), while $\mathrm{B}_{\mathrm{x}}^{4}$ is simply given by a set of bars placed over and under the NIO1 front vacuum chamber, in the limited space available. Note that $\mathrm{B}^{4}$ gives a large fringing field in the driver, but it is easily removable and is uniform on the axis of the 9 extracted beamlet; on the contrary $\mathrm{B}^{3}{ }_{\mathrm{x}}$ is weaker on the central beamlet axis (and it require a source shutdown for changes) but gives negligible fringing field. The sum of these terms (configuration 'f3') temperates these drawbacks, and most of all, total strength can be changed by only $\mathrm{I}_{\mathrm{pg}}$ current reversal: best $\mathrm{H}^{-}$result are obtained for $\mathrm{I}_{\mathrm{pg}}=-300$ or $-400 \mathrm{~A}$, with $\mathrm{B}_{\mathrm{x}}^{\mathrm{s}}=-12 \mathrm{mT}$, almost equally shared by the 3 systems described. It may be argued that this large value is specific to NIO1 source geometry (and to Cs-free operation) and larger sources will probably follow the Larmor radius to size ratio scaling.

In perspective, an important issue is the interference of $\mathrm{B}^{\mathrm{s}}$ with the deflecting field $\mathrm{B}^{\mathrm{d}}$ $[111,112]$ in the acceleration column, especially for their influence on voltage breakdowns (typically limiting NIO acceleration voltage $\mathrm{V}_{\mathrm{t}}<25$ $\mathrm{kV}$ for reason to be clarified).

\subsubsection{Beam lateral cameras as effective diagnostic}

An immediate monitor of $\mathrm{H}^{-}$beam is given by lateral cameras CAM1 and CAM2, with view direction shown in fig 20a: the $3 \times 3$ matrix of NIO1 beamlets projects in a three apparent macrobeamlet on each cameras $[118,120]$. The perspective distortion is small within macrobeamlet and noticeable for vacuum chamber wall (at $\mathrm{r}=0.175 \mathrm{~m}$ where $\mathrm{z}$ is beam axis, $\mathrm{x}$ is the vertical axis, and other Cartesian and cylindrical coordinates defined as usual). Luminosity can be taken roughly proportional to projection of beamlet relative density $\mathrm{b}(\mathrm{x}, \mathrm{y}, \mathrm{z})$, apart where obvious reflections appears (walls in view area are covered by black foil). A first information is average spacing between beamlets, about $14 \mathrm{~mm}$ at PA and REP exit and slowly increasing afterwards, as sampled at 3 positions $[119,120]$; beam width and divergence are similarly obtained.

Special algorithms to give an unbiased reconstruction of $b(x, y)$ at given $z$ are fully discussed in Ref. [120], as well variation with z. As regards to experimental upgrades, this well motivates the installation of other cameras at different $z$ (near position A in fig. 20a).

\subsubsection{Transient suppression and gas conditioning}

As noted in [118] based on $\mathrm{H}^{-}$beam data for Csfree operation collected between 2015 and August 2019 , in steady state regimes exceeding few hours observation time (directly studied only in NIO1 worldwide), the $\mathrm{H}^{-}$extracted current has slow transients (with 10 minutes typical time scale), inversely correlated to plasma luminosity and to coextracted electron current, whose evolutions (at constant source pressure, rf power, and electrode voltages) were simply witnessed by the signals: $\mathrm{V}_{\mathrm{pmt}}$ is the signal from a photomultiplier (PMT) looking axially (in z direction) from one CF16 in the NIO1 backplate; its line of sight (see Fig 19) cross all the driver; $\mathrm{I}_{\mathrm{cfc}}$ is the signal from a carbon fiber composite (CFC) tile, which now mounted about position A in Fig 20. An example of $V_{\text {pmt }}$ and $\mathrm{I}_{\mathrm{cfc}}$ and the conditions used (for the total source acceleration absolute voltage $\mathrm{V}_{\mathrm{t}}$ and the extraction voltage $\mathrm{V}_{\mathrm{e}}$ ) is given in fig. 21, previous data were shown in Fig 9.c of Ref 116. The Fig 21 data shows an overall factor 10 improvement in $\mathrm{I}_{\mathrm{cfc}}$ respect to the cited figure, in some part due to small increments in RF power $P_{k}$ and source pressure $p_{s}$, in some part due to evolved filter configuration; moreover the Pyrex window was changed, and lower values of $\mathrm{V}_{\mathrm{pmt}}$ were observed (minimum is now $0.75 \mathrm{~V}$ instead of $0.9 \mathrm{~V}$ ).

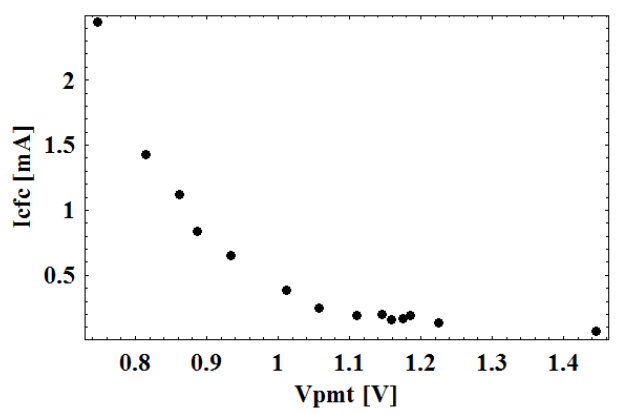

Fig. 21 Example of inverse correlation of beam current $I_{\mathrm{ccc}}$ vs plasma luminosity signal $\mathrm{V}_{\mathrm{put}}$ during a slow transient at control parameter fixed: $\mathrm{P}_{\mathrm{k}}=1.3 \mathrm{~kW}, \mathrm{p}=0.9$ $\mathrm{Pa}$, filter current $\mathrm{I}_{\mathrm{p}}=400$ A with configuration ' $\mathrm{f} 3$ '; beam 
voltage reduced at $\mathrm{V}=4 \mathrm{kV}$ (extraction voltage $\mathrm{V}=0.45$ $\mathrm{kV}$ ) for better stability.

Both transient to high current from a low current (high luminosity) base state and vice versa were observed (consistently with multi-stability introduced before). We noted that: improving air cooling of RF windows helps, to promote transient to high $\mathrm{I}_{\mathrm{cfc}}$ current; and Pyrex condition matters. But most of all using the source with oxygen (with $10 \%$ Ar for spectroscopy purposes) as a feeding (or process) gas for one day helps $\mathrm{H}^{-}$yield in following days (consistently with the fact that NIO1 was operated with air in 2014): transient disappeared allowing a few days (at least two) of productive source operation for parametric scans (of perveance, filter and bias).

Among heuristic and partial explanations, it may be proposed that an increase of plasma driver $\mathrm{T}_{\mathrm{e}}$ (say from 4 to $5 \mathrm{eV}$ ) increases plasma luminosity; simulations show that the protection offered by filter to $\mathrm{H}^{-}$in extraction region is much weaker with the larger $T_{e}$, which may explain lower current. In other words, the ideal driver plasma must have density $\mathrm{n}_{\mathrm{e}}$ as large as possible and $\mathrm{T}_{\mathrm{e}}$ just enough (4 eV) to sustain ionization, so that a colder extraction plasma is possible; increasing RF power $\mathrm{P}_{\mathrm{k}}$ may well give an increase of the $\mathrm{n}_{\mathrm{e}} \mathrm{T}_{\mathrm{e}}$ product, while a SEE increases more directly increase $n_{e}$, showing a possibility for surface conditions to affect source operation (even in a Cs-free operation). Also position of $\mathrm{H}_{2}$ input gas may affect driver operation.

The simpler experiment anyway was to change conditioning gas [118]. A campaign including exposure to different gases (namely Ar, $\mathrm{N}_{2}$, a mixture of $\mathrm{O}_{2}$ with $5 \% \mathrm{Ar}$, and $\mathrm{Xe}$ ) interleaved with pure $\mathrm{H}_{2}$ runs was performed in OctoberNovember 2019, to put chemical and physical effect (and differences with air) into evidence; campaign was begun by taking 3 days of $\mathrm{H}^{-}$beam data as a reference set after the source was operated as long enough as possible to make effect of previous conditioning negligible. Then one day of conditioning and two days of $\mathrm{H}_{2}$ process gas were alternated. In first instance $\mathrm{H}^{-}$current $\mathrm{I}_{\mathrm{cfc}}$ (see Fig 21 ) is collected by a carbon fiber composite (CFC) tile, with a multiplying factor dependent on gas pressure $\mathrm{p}_{2}$ in the vessel. The $\mathrm{H}^{-}$current can also be estimated from power supply current (corrected for dark current at plasma off) $\mathrm{I}_{\mathrm{a}}$, with resulting error about $\pm 0.3 \mathrm{~mA}$. For few selected cases, calorimetric data $\mathrm{I}_{\mathrm{cal}}$ inferred by $\mathrm{CFC}$ temperature rise seen by an IR camera are also available (see later). Lower vessel pressure point $\mathrm{p}_{2}<0.04 \mathrm{~Pa}$ are obtained with cryogenic pumps on. When cryopumps are off for regeneration, $\mathrm{p}_{2}$ is larger (and $\mathrm{p}_{\mathrm{s}}=3.5 \mathrm{p}_{2}$ from known conductance and turbopumps speed), so that $\mathrm{I}_{\mathrm{cfc}}$ is strongly amplified by collection of secondary particles.

Preliminary results show that the $95 \%$ oxygen mixture is slightly better (for $\mathrm{H}^{-}$conditioning) than nitrogen alone, and they surpass argon; xenon (whose mass is similar to Cs) is somewhat in between. After each gas change, no direct presence of the previous gas was observed (neither on emission spectroscopy nor on current evolution), apart in the first 2 hours of operation in some cases. Relative trends at fixed pressures $\mathrm{p}_{2}<0.05 \mathrm{~Pa}$ and $\mathrm{P}_{\mathrm{k}}=1400 \mathrm{~W}$ indicate the merit of conditioning procedures: result ranges from $\mathrm{I}_{\mathrm{cfc}}=2 \mathrm{~mA}$ maximum for the reference set to $\mathrm{I}_{\mathrm{cfc}}=4.5 \mathrm{~mA}$ after argon to $6 \mathrm{~mA}$ after nitrogen to $\mathrm{I}_{\mathrm{cfc}}=8 \mathrm{~mA}$ after oxygen to $7 \mathrm{~mA}$ after Xenon. Comparison with $\mathrm{I}_{\mathrm{a}}$ is well in progress [123] and generally confirms the previous ordering; data $\mathrm{I}_{\text {cal }}$ (time consuming to take, it requests very stable source condition for 10 minutes) are available only for a few cases for the $95 \% \mathrm{O}_{2}$ conditioning procedure only; in the same case of before we have $\mathrm{I}_{\mathrm{a}}=7 \mathrm{~mA}\left(\mathrm{I}_{\mathrm{cal}}=3 \mathrm{~mA}\right)$.

Difference of $\mathrm{I}_{\mathrm{a}}, \mathrm{I}_{\mathrm{cfc}}$ and $\mathrm{I}_{\mathrm{cal}}$ mainly depends on pressure $\mathrm{p}_{2}$. Considering now the $\mathrm{p}_{2}>0.05 \mathrm{~Pa}$ range we note much higher $\mathrm{I}_{\mathrm{cfc}}$ currents and also some improvement also on $\mathrm{I}_{\mathrm{a}}$, reaching $\mathrm{I}_{\mathrm{cfc}}=23 \mathrm{~mA}$ $\left(\mathrm{I}_{\mathrm{a}}=10.5 \mathrm{~mA}\right)$ after oxygen and $24 \mathrm{~mA}$ after xenon $\left(\mathrm{I}_{\mathrm{a}}=10.5 \mathrm{~mA}\right)$; the calorimetric current is very modest (possibly for effect of gas cooling and IR absorption to be quantified). Fig. 22 beam orthogonal projection images shows that a good balance of 9 beams is possible at lower $V_{t}$ while uniformity worsen at higher $\mathrm{V}_{\mathrm{t}}$. To ameliorate this, the BP mask aperture is being enlarged (more plasma on side beamlets).

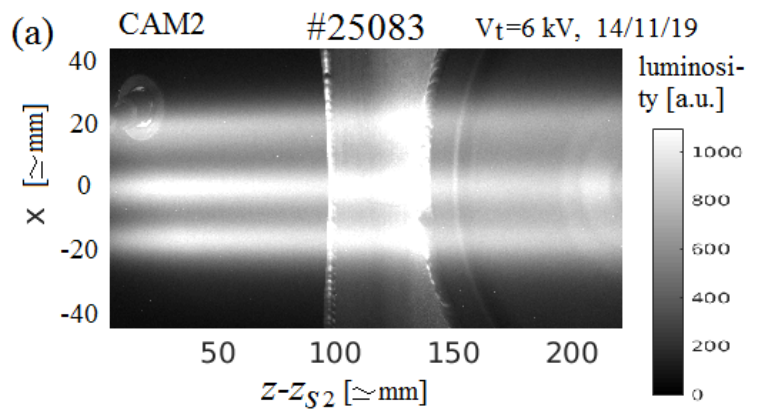




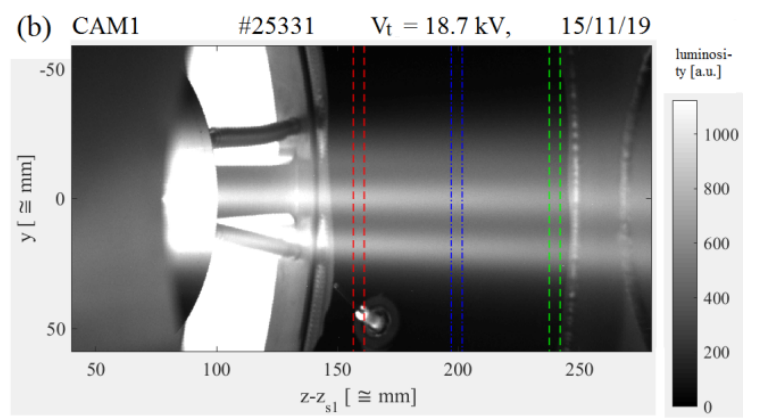

Fig. 22 Lateral views of $\mathrm{H}^{-}$beamlets (cases after Nitrogen conditioning): a) Dataset number 25083, $\mathrm{V}_{\mathrm{e}}=0.5 \mathrm{kV}, \mathrm{I}_{\mathrm{a}}=2.8 \mathrm{~mA}$, view CAM2 (looking towards y); b) Dataset number 25331, view CAM1 (looking towards $+\mathrm{x}$ ), $\mathrm{V}_{\mathrm{e}}=2.05 \mathrm{kV}, \mathrm{V}_{\mathrm{t}}=18.7 \mathrm{kV}, \mathrm{I}_{\mathrm{a}}=9.3 \mathrm{~mA}, \mathrm{I}_{\mathrm{cfc}}=5$ $\mathrm{mA}$. Note bands where relative current profiles are measured.

\subsection{RAID reactor at EPFL}

The Resonant Antenna Ion Device (RAID) at the Swiss Plasma Center is a linear $(1.9 \mathrm{~m}$ total length, $0.4 \mathrm{~m}$ diameter) basic plasma physics device equipped with a set of radio frequency resonant network antennas delivering up to $10 \mathrm{~kW}$ at 13.56 $\mathrm{MHz}$, producing steady-state helicon plasmas of different gases. A schematic of RAID with a few diagnostics is shown in Fig. 23 and a detailed description can be found in Ref. [125]. The plasma in RAID is sustained by a helicon wave generated by a novel birdcage antenna with cosine azimuthal current distribution [126]. The resonant birdcage antenna maintains a stable intense plasma column from low $(300 \mathrm{~W})$ to high $(10 \mathrm{~kW})$ steady-state RF power in hydrogen/deuterium or argon, enabling plasma diagnostic measurements over the whole volume of the device. The plasma density, $10^{18}$ to $10^{19} \mathrm{~m}^{-3}$, and uniform static magnetic field, 200 to $800 \mathrm{G}$, are typical of high density, low B-field helicon sources where the RF excitation frequency, 13.56 MHz, is far above the ion cyclotron frequency and much lower than the electron cyclotron and plasma frequencies. RAID is suitable for studying the physics of helicon-generated plasmas for negative ion beams for fusion because its plasmas are characterized by a significant fraction of negative ions (of the order of a few $10^{16} \mathrm{~m}^{-3}$ ), produced in the volume, i.e. in the absence of caesium, and at low neutral gas densities (of the order of $0.3 \mathrm{~Pa}$ ). The present contribution summarizes the results obtained in RAID in the past years, which broaden our comprehension of hydrogen/deuterium plasma discharges in helicon regimes in the presence of negative ions.

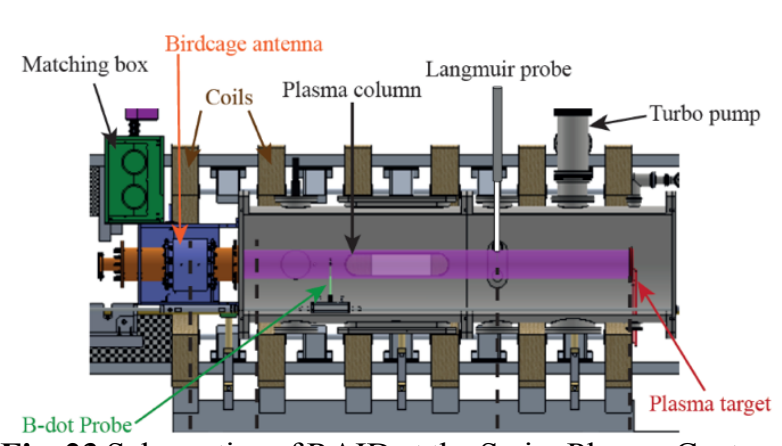

Fig. 23 Schematics of RAID at the Swiss Plasma Center.

\subsubsection{Production of negative ions by volume processes}

First indications of the presence of negative ions in helicon plasmas in RAID were obtained using passive optical emission spectroscopy [80]. Motivated by these findings, we recently used a combination of cavity ring down spectroscopy (CRDS) and Langmuir probe laser photodetachment (LPLP) to perform local measurements of negative ion density with a spatial resolution of a few $\mathrm{mm}[127,128]$. The LPLP system consists of a vertically movable LP at different radial locations inside the plasma column. The photodetachment signals are measured with an AC toroidal current transformer triggered by a fast photodiode. The theory of LPLP requires the knowledge of the local value of the electron density, which is usually measured by the same probe measuring the photodetached current. However, owing to the non-negligible electron magnetization in RAID, the Langmuir probe sweeps cannot be relied on to compute the local electron density. To overcome this problem, we have used the line-integrated negative ion density measured using CRDS for absolute calibration, which is not affected by the presence of the DC magnetic field. The negative ion density profiles obtained with laser photodetachment are interpolated using Gaussians normalized to the maximum data value. Thus, they can be still used to extract the width and the position of the negative ion shell. By combining LPLP normalized profiles and the line- integrated negative ion density measured by CRDS the radial absolute negative ion density profiles (shown below) can be calculated. Results in hydrogen and deuterium plasma as a function of the injected RF power are shown in Fig. 24. We have measured a ratio of negative ion density over electron density of about 0.3 in hydrogen and 0.05 deuterium at the edge of the plasma column, where the largest density of negative ions is expected. 

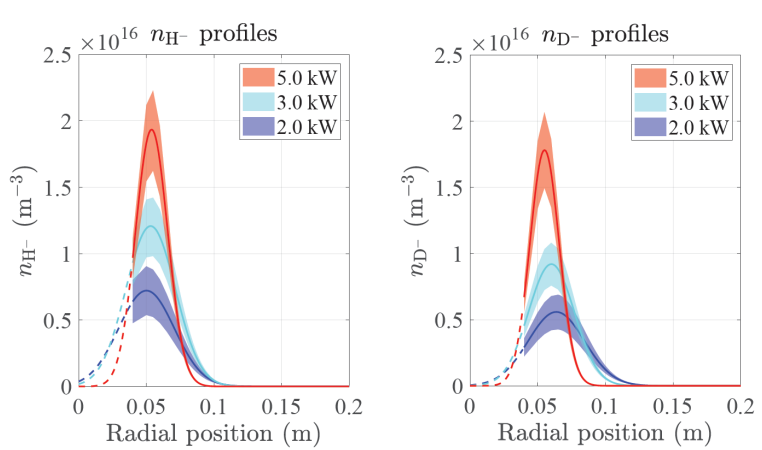

Fig. 24 Radial profiles of negative ions in $\mathrm{H}_{2}$ and $\mathrm{D}_{2}$ plasmas for different powers obtained using the relative negative ion profiles measured with laser photodetachment and calibrated with CRDS line-integrated measurements.

\subsubsection{Helicon waves in RAID}

Although the production of an elongated plasma column and the observation of the argon blue core in RAID are signatures of the propagation of a helicon wave, a direct experimental verification of the existence of helicon waves is required. A threedimensional magnetic field (B-dot) probe was developed to characterize the structure of the helicon waves produced by the birdcage antenna [126]. The B-dot probe measures the three components of the oscillating magnetic field associated to the helicon wave and can be automatically displaced along the plasma column. To absolutely calibrate the B-dot probe, the presence of an external time varying magnetic field of known intensity is required. For this, a dedicated calibration test bed was developed.

The steady-state plasma column is radiallyunbounded and typically $1.5 \mathrm{~m}$ long. It can be conveniently measured in the main vacuum chamber, axially and radially, by using the B-dot probe [129] as shown in Fig. 25. These results show the first formal proof of helicon wave generation by a resonant birdcage antenna [126]. The observed dependence of axial wavelength on the plasma density and external DC magnetic field is smooth and continuous, showing no preferred dependence on the antenna length, nor the standing wave structure. Furthermore, heavy or light ions $\left(\mathrm{Ar}^{+}\right.$or $\left.\mathrm{H}_{2}^{+}\right)$had no notable influence on the axial wavelength, which depended only on the electron density and the DC magnetic field as expected from helicon theory. The measured axial wavelengths were compared with semi-analytical helicon theory and a purely numerical model. The experimental data and the calculated $m=1$ eigenvalue of lowest axial wavelength were in good agreement, using no fitted parameters [126]. Numerical calculations of the self-consistent currents in the antenna and the plasma spontaneously converged towards this same dominant mode. In conclusion, the birdcage helicon source presents a new alternative to conventional partial-helix antennas, with advantages of wider parameter space and improved plasma stability, especially for light ion (hydrogen) plasmas.

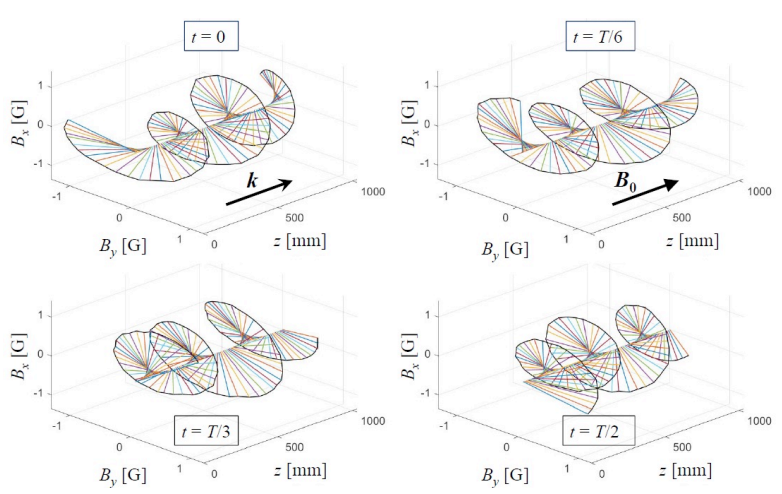

Fig. 25 The transverse magnetic field raw data, measured on axis, for three equal time intervals during a RF half-period ( $t=0, T / 6, T / 3, T / 2)$. The magnetic field spatial structure is seen to be a left-handed helix, rotating clockwise with respect to the direction of $\mathrm{B}_{0}$, in accordance with helicon theory. Hydrogen plasma at 0.3 $\mathrm{Pa}$, magnetic field $200 \mathrm{G}$, RF power $1.5 \mathrm{~kW}$.

\subsubsection{First negative ion extraction experiments}

In the past year, we manufactured a negative ion extractor for RAID and performed first tests. Among two extraction geometries, we chose to investigate radial extraction through an axial slit along one radial direction, compatible with a photoneutralizer, which was the original motivation of RAID development. The radial extractor has the notable advantage of having an intrinsic magnetic filter field, required for the propagation of the helicon waves and thus to sustain the plasma discharge. Fig. 26 shows a picture of the radial extractor, which was manufactured and installed in RAID in the period covered by this report. The volume-produced negative ions are extracted from the plasma volume and they are accelerated through a $1 \mathrm{~mm}$ gap between the plasma grid and extraction slits and impinge on a bulky copper collector located 13.4 $\mathrm{mm}$ downstream the extraction slit. Fig. 26 shows the dependence of the three currents on the extraction voltage, which was scanned up to $1 \mathrm{kV}$. During a first experimental campaign, we tested the extractor in the configuration that exhibits an annular distribution of negative ions at approximately $\mathrm{r}=4.5 \mathrm{~cm}$ from the axis of the plasma column. The plasma grid was positioned at $\mathrm{r}=6 \mathrm{~cm}$ and the currents on the plasma grid, the extractor and the collector were measured.

The current measured on the extraction grid is mostly due to electrons transported by ExB drift. 
The current at the collector is mostly carried by negative ions (due to the geometric design of the extractor, which filters out the electrons) and shows an almost linear dependence, indicating that the extractor is operated below the Child-Langmuir limit.

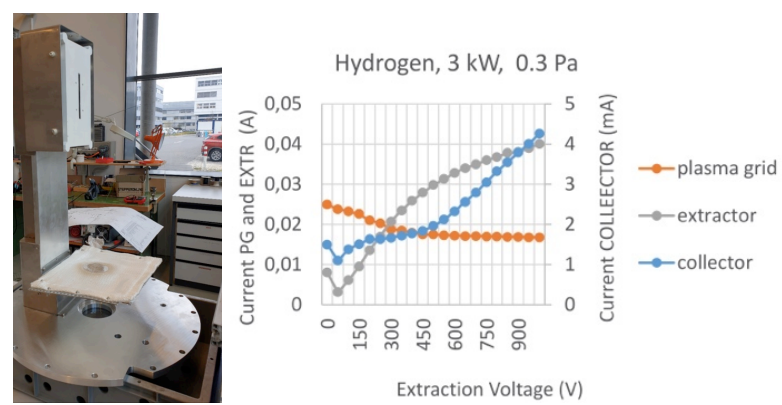

Fig. 26 Left: The radial extractor for RAID. Right: First extraction results.

\section{Neutral Beam Injector (NBI) Challenges}

Kinetic plasma modeling of a fusion-type negative ion source brought some physical insight which when combined with experimental measurements did improve significantly the understanding of these devices. Models provided trends similar to the ones observed in the experiments but also some detailed analysis of the ion source plasma properties which would otherwise be accessible experimentally with difficulty. In addition, due to the complex nature of NIS (interplay among different subsystem) very useful mathematical tools have been recently developed to identify drivers key elements for optimized NIS performances. In this section, the two numerical approaches will be presented.

\subsection{Latest improvements in Numerical modelling of negative ion sources for NBI}

A significant effort has been devoted in the past ten years to model the plasma behavior of fusion type NIS with fluid [130-132] and Particle-In-Cell models with Monte-Carlo-Collisions (PIC-MCC) [63,133-136]. The latter have been optimized to run on supercomputers via a parallelization of the algorithms using MPI and OpenMP libraries. The algorithm is time explicit and electrostatic; the electric field is calculated by solving Poisson's equation on a mesh while the magnetic field profiles are prescribed. The simulation of the ion source plasma is often performed by replacing the vacuum permittivity $\varepsilon_{0}$ in Poisson's equation by a given value $\varepsilon$. This assumption is equivalent to modelling a plasma density smaller by a scaling factor $\alpha=\sqrt{\varepsilon / \varepsilon_{0}}$ while allowing to artificially consider the real plasma densities for the calculation of the collisions between charged particles. The plasma sheath is hence larger by a factor $\alpha$ which does not have any consequences on the plasma behaviour as long as:

(1) the plasma is quiescent or without any enhanced transport due to instabilities with frequencies and growth rates related to the vacuum permittivity;

(2) the particle kinetics inside the plasma sheath are preserved. In such situations, the plasma profiles are independent of the amplitude of the external power of the discharge [133].

The question of the artificial magnetization of the plasma sheath and its effect on the quasi-neutral plasma volume is still an open problem.

\subsubsection{Plasma properties of ITER-like negative ion sources}

PIC-MCC models have be used to analyze the effect on the plasma properties of the magnetic filter field in the ion sources expansion chambers. Gas dissociation and ionization occur mainly inside the discharge (also called driver), region where the Radio-Frequency (RF) power is absorbed and coupled to the plasma while it is almost non-existent inside the volume covered by the magnetic filter field as the electron temperature is significantly below the ionization threshold. There is hence a flow of charged particles from the discharge toward the expansion chamber. Electrons are strongly magnetized (while ions are not) and will drift inside the magnetic field. The drift is directed toward one of the lateral surfaces of the ion sources (depending on the sign of the magnetic field) which results in a transverse polarisation of the plasma as a consequence of the restoring force between particles of opposite charges to keep the plasma quasi-neutral. The polarisation induces a transverse asymmetry in the plasma parameters which has been observed both in the models [133-136] and in the experiments [98]. Fig. 27 shows the 2D electron temperature profile for the case of the half-size ITER prototype source ELISE. The simulation is performed in 2D including particle losses in the third dimension calculated analytically for the ions (a loss frequency deduced from the Bohm velocity) while the electrons are removed from the simulation domain if their kinetic energy along that direction exceeds the difference between the wall and plasma potentials [133] (thin sheath approximation), respectively. The calculation corresponds to the plane perpendicular to the magnetic filter field lines, i.e., where the particle drift occurs. We assumed an absorbed power of $120 \mathrm{~kW}$ per driver (which occupies all the space in the third direction), dimensions of $40 \mathrm{~cm}$ in length and $1 \times 0.9 \mathrm{~m}^{2}$ of transverse cross section. The 
magnetic field strength is $30 \mathrm{G}$ on the $\mathrm{PG}, 18 \mathrm{G}$ at the discharge exit and $8 \mathrm{G}$ at its centre (sufficiently to magnetize the electrons). The numerical resolution is $1024 \times 3072$ grid nodes and 40 particles per cell with a mesh size on the order of the electron Debye length to avoid any numerical instabilities. The vacuum permittivity is increased artificially by a factor 400 (i.e., a plasma sheath $\alpha=20$ times larger) to model an ITER-like volume averaged plasma density of $5 \times 10^{17} \mathrm{~m}^{-3}$. The transverse polarization of the plasma due to the electron magnetic drift results in an asymmetric electron current on the plasma grid, as well as an asymmetry in the plasma potential, particle temperatures and densities [136]. The production of negative ions via electron inelastic collisions with the background gas inside the plasma volume is hence strongly affected by the asymmetry as the process is two-fold:

(1) the hydrogen-isotope gas is first excited vibrationally in a region where the electron temperature is of the order of $10 \mathrm{eV}$ (i.e., typically in the discharge);

(2) the negative ions are generated as the byproduct of DA of vibrationally excited molecules in a location where the electron temperature is $\sim 1$ $\mathrm{eV}[71,137]$. In fusion-type ion sources, the vast majority of negative ions are produced on the PG via the impact of neutrals and ions with the caesiated surface. According to estimations, a negative ion current as large as $600 \mathrm{~A} / \mathrm{m}^{2}$ on the PG may be expected from the flux of neutrals [138], which generates a virtual cathode at that location. The depth of the potential well is strongly modulated by the flux of positive ions generated in the discharge. The question of the asymmetry of the extracted beamlet current density is currently an open problem.

PIC-MCC models have also demonstrated alongside experimental observations the effect of biasing the PG with a voltage greater than the ion source wall potential (assumed grounded hereafter). The bias voltage does not modify the amplitude of the plasma potential in the discharge as it is screened by the magnetic filter field. As a consequence, the shape of the potential profile may be controlled in the extraction region. The profile is flattened with a reduced sheath potential when the PG is floating [97,133]. A larger bias voltage, such that the PG bias current is negative (i.e., the current carried by negative charges is greater than the one from the positive charges), will generate a potential well which is beneficial for the extraction of negative charges. In addition, the reduction of the sheath voltage results in a lower co-extracted electron current as the latter are collected on the PG instead. Note that the production of negative ions by positive ion impacts is found to be negligible in the calculations when the PG is floating [139]. Most ions (about $70 \%$ ) are generated by $\mathrm{H}$ atom conversion on the caesiated surface.

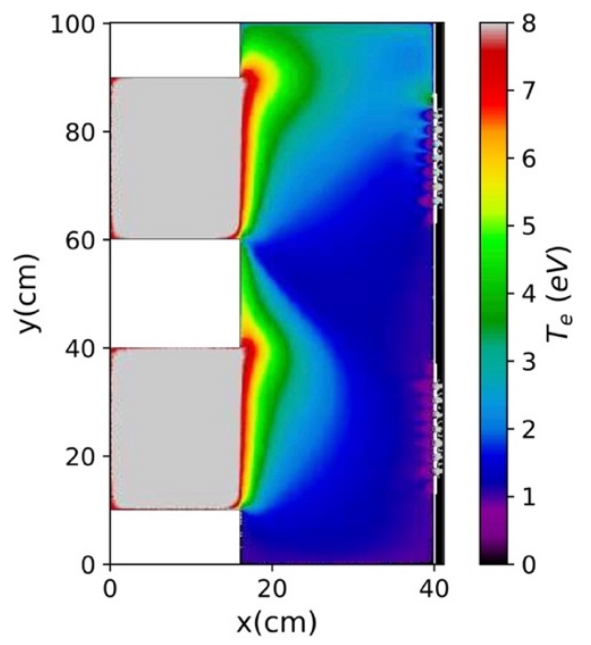

Fig. 27 2D Particle-In-Cell calculation of the electron temperature profile for the half-size ITER prototype ELISE (Max Planck Institute, Garching, Germany). The magnetic field is perpendicular to the simulation domain. Particle losses in the third dimension are accounted for.

In addition, the low-pressure working conditions $(0.3 \mathrm{~Pa})$ of the ITER-prototype ion source results in a depletion of the neutrals. A significant difference between the temperature of the atomic hydrogen population $(\sim 1 \mathrm{eV})$ compared to the molecules $(\sim 1000 \mathrm{~K})$ was also observed in the experiments [140]. Neutrals were modeled as particles in the PIC algorithm but with a different time step and weight compared to the charged particles as the neutral density is about $5 \times 10^{19} \mathrm{~m}^{-3}$ for $\mathrm{H}_{2}$ and typically five times less for the atoms. Neutral dynamics requires millisecond-scale time integration to converge while the plasma reaches steady-states in a couple tens of microseconds (which is related to the time scale of the positive ions). This justifies the implementation of different time scales (which greatly accelerate the convergence time of the algorithm) as neutral profiles evolves slowly in contrast with the ions. Increasing the RF power in the model induce a lessthan-linear increase of the electron density due to the neutral depletion) which saturates at high power. In that case, a larger RF power results in an increase of the electron temperature and plasma potential amplitude instead of the plasma density. The power saturation threshold is shifted toward larger densities for higher background gas pressure. A calculation without magnetic field and for the BATMAN prototype source found a 
threshold at $30 \mathrm{~kW}$ for $0.15 \mathrm{~Pa}$ and

$>100 \mathrm{~kW}$ for $0.3 \mathrm{~Pa}$ [133], respectively. The main pathway for the energy gain of hydrogen atoms is through the recombination of positive ions at the ion source walls while the main loss mechanism is by colliding with surfaces at low pressure. This explains the $\mathrm{eV}$-scale temperature of the atoms as the positive ions are generated mostly inside the discharge and hence impact the grounded walls with a large kinetic energy after being accelerated by the ambipolar electric field. Their distribution function is non-Maxwellian in the model [133]. Lastly, the main source of power for the hydrogen molecules is due to elastic collisions with the atoms while power losses originates from backscattering against surfaces. Modeling neutral dynamics in low pressure plasma sources is hence highly dependent on the physical mechanisms associated with particle surface interactions. Unfortunately, at the moment, most particle-wall coefficients (particle reflection, accommodation, recombination) are unknown.

\subsubsection{Negative ion extraction}

Modelling the transport and generation of negative hydrogen isotope ions on the PG requires a simulation domain which is limited to a small volume in front of a given aperture. In 2D, the top and bottom boundary conditions are periodic which implies that an infinite array of apertures are modelled. The plasma is generated in a simplified manner by re-injecting an electron-ion pair on the left-hand-side (LHS) of the simulation domain, inside a given volume, for each positive ion lost on the physical surfaces. This mimics the ionization process and keep the plasma density constant on average. The latter being an external parameter to the model. In addition, the electron temperature is maintained at a fixed value inside the particle injection region by periodically replacing the velocity of the electrons by a new one drawn from a Maxwellian distribution [133]. The negative ion production rate on cesiated surfaces deduced from the atomic flux [138] (a density of $\sim 10^{19} \mathrm{~m}^{-3}$ and temperature of $\sim 1 \mathrm{eV}$ ) together with the conversion probability [141] is estimated to yield a current density of about $600 \mathrm{~A} / \mathrm{m}^{2}$ on the PG. The negative ion density is as a result space charge limited near the grid and a potential well (a virtual cathode) is present. The latter implies that the current crossing the well is strongly reduced by its depth [138] and the potential profile must consequently be well resolved by the numerical mesh. There is typically an ion-ion plasma in front of the grid surface with a negative ion to electron density ratio of a factor 5-10 at the location of the potential well. The effect of the negative ion population on the well profile is currently an open question.
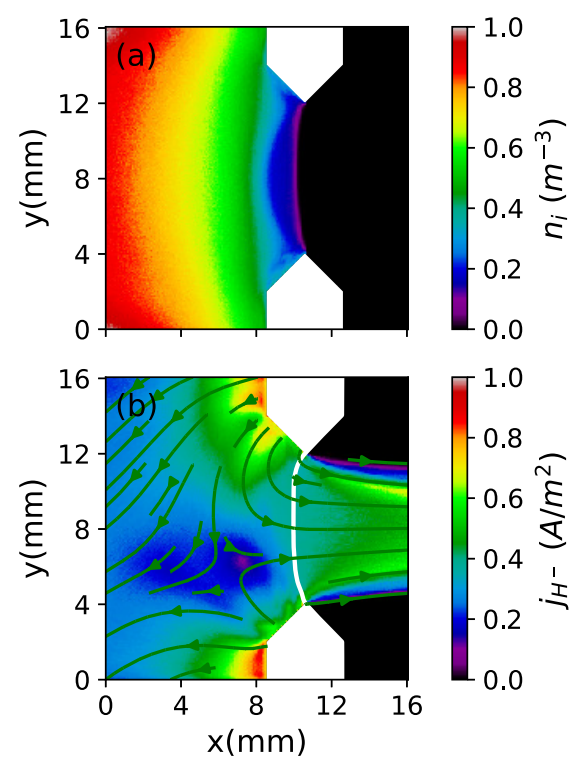

Fig. 28 2D normalized plasma density (a) and negative ion current density (b) profiles in front of a chamfered slit aperture. 2D PIC-MCC calculation. The maximum plasma density is $2 \times 10^{17} \mathrm{~m}^{-3}$ and the ion current density $225 \mathrm{~A} / \mathrm{m}^{2}$, respectively. The current streamlines are shown in green and the plasma meniscus in white.

The size of the simulation domain of Fig. 28 is $32 \times 16 \mathrm{~mm}^{2}$ in $2 \mathrm{D}$ (Fig. 28 displays a portion of the domain) and a numerical mesh of $4096 \times$ 2048 nodes is necessary to properly resolve the plasma, beam and virtual cathode properties for an average plasma density of $3 \times 10^{17} \mathrm{~m}^{-3}$. The electron temperature is $2 \mathrm{eV}$ and there is no scaling in this calculation. Fig. 28 shows the 2D normalized a) plasma density (maximum is $2 \times$ $10^{17} \mathrm{~m}^{-3}$ ) and b) negative ion current density (maximum is $225 \mathrm{~A} / \mathrm{m}^{2}$ ). The negative ion current streamlines are also shown together with the plasma meniscus (which separates the plasma from the void of the accelerator vessel). There is a cusp magnetic field surrounding the slit aperture in the xy plane with a strength of $140 \mathrm{G}$ on the front PG surface and another component, perpendicular to the simulation domain with an amplitude of $75 \mathrm{G}$ [133] (i.e., the magnetic filter field of the expansion chamber). There are no negative ions produced inside the plasma volume in this calculation (only on the PG surface). The negative ion current density profile is asymmetric due to the slight magnetization of the ions. The $\mathrm{x}$-point visible on the current streamlines is shifted off axis which is consistent with experimental measurements [142]. The ratio of the extracted ion current originating from the chamfered surface compared to the flat side facing the plasma (see Fig. 28) is $\sim 2.2$ while the surface area ratio is $\sqrt{2}$ indicating that the 
proximity of the accelerator vessel slightly increases the extraction probability on the chamfered side. Furthermore, the plasma density in Fig. 28a extends up to the tip of the chamfered surface. The positive ion density reduces the depth of the potential well and as a result increases the negative ion production yield. The latter are hence extracted from the plasma, across the meniscus, for working conditions such that the beamlet are in socalled perveance match. The virtual cathode depth is about $-1.2 \mathrm{~V}$ near the front side and $-1.8 \mathrm{~V}$ close to the tip. As a general result, the model shows that the extracted negative ion beamlet current is somewhat proportional to the surface area of the aperture irrespective of its geometry. Similar conclusions were drawn from the experiments [143]. The question of the possible degradation of the beam emittance in the case of a chamfered aperture configuration is currently an open problem. The main drawback of the calculation shown in Fig. 28 is the large number of numerical mesh nodes required for convergence which implies that it would not be feasible in 3D. An alternative approach is to scale down the plasma density together with the negative ion current density emitted on the PG. This relaxes the constrains on the grid spacing. Calculations show that scaling down the plasma density by 16 for instance (hence a mesh four times larger, resulting in a computational time gain of a factor 256 in 3D) provides a beamlet profile with similar features for the same perveance except when aberrations are presents as a larger mesh tends to smooth them out $[133,144]$. Note that the accelerator grid voltages must also be scaled down according to the ChildLangmuir law.

\subsubsection{Production of negative ions in the plasma volume of a linear device.}

In this paragraph we present the numerical model of RAID NIS (Sect. 3.3).

Fig. 29 shows the electron temperature and negative ion density profiles modelled in $2 \mathrm{D}$ with a PIC-MCC. The ion source has a diameter of 40 $\mathrm{cm}$, a length of $1.5 \mathrm{~m}$ and a magnetic field strength of $200 \mathrm{G}$ which is sufficient to magnetized both the electrons and somewhat the ions in hydrogen. RF power is $3.1 \mathrm{~kW}$ and the background gas pressure is $0.3 \mathrm{~Pa}$. The simulation is performed in $2 \mathrm{D}$, in the plane perpendicular to the magnetic field lines, with particle losses in the third dimension also accounted for (see the preceding sections). The advantage of such a configuration is that the charged particle drift in the magnetic field is a closed loop and hence the plasma has a cylindrical symmetry.

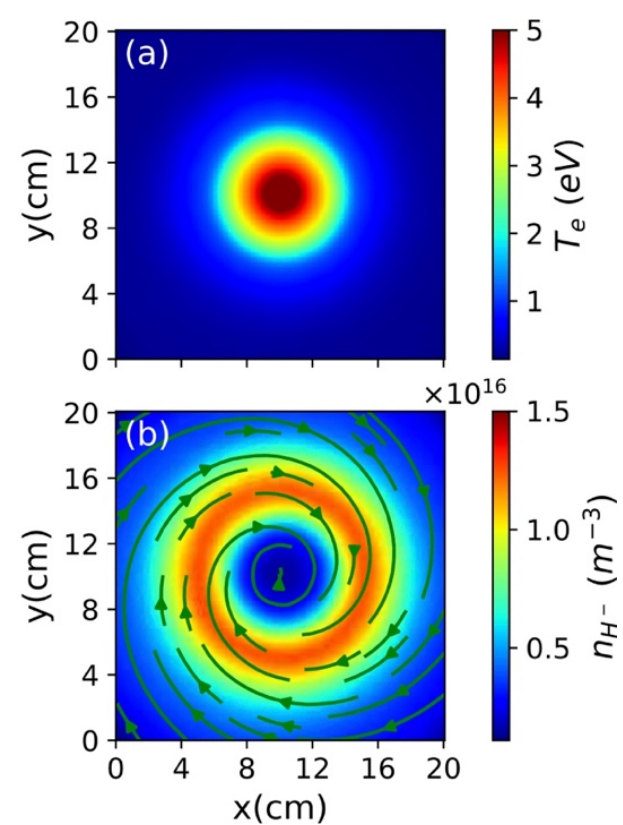

Fig. $292 \mathrm{D}$ electron temperature (a) and negative ion density (b) profiles in the RAID linear device. 2D PICMCC calculation. The current streamlines are show in green. The magnetic field lines are perpendicular to the simulation domain. The background gas is hydrogen.

The region of the helicon power deposition is correlated with the high electron temperature in Fig. 29a while the negative ion density in Fig. 29b peaks where the temperature falls below $1 \mathrm{eV}$. This is characteristics of the two-step process associated with the vibration excitation and dissociative attachment of the hydrogen isotope molecules [71]. The ring-like profile of Fig. 29b (maximum of $1.3 \times 10^{16} \mathrm{~m}^{-3}$ ) has also been observed in the experiments [80]. In addition, the residence time of the electrons and hence the negative ion production yield may be increased by biasing negatively the end-plates (short-circuiting the magnetic field lines at both ends of the cylinder). Electrons are strongly attached to a magnetic field line and will stay confined for a longer time. Linear devices may consequently be used to extract negative ions across a slit aperture embedded on the cylinder wall but the extraction voltage from the accelerator will generate an asymmetry in the plasma parameters. The question of extracting a negative ion current similar to the one of the conventional ITERprototype plasma sources is currently an openproblem as in the case of the latter the ions are produced directly on the surface near the aperture. A negative ion density on the order of $10^{17} \mathrm{~m}^{-3}$ with a ring radius similar to the one of the linear device might be required.

As a general statement to avoid the plasma asymmetry in ion sources with a magnetic filter perpendicular to the particle flow from the discharge, a solution would be to have a race-track 
like geometry in the transverse direction (similar to an elongated cylinder) with a magnetic field normal to the walls in that plane and for instance a flat antenna on one end of the main axis such that the charged particle flow will have a closed (transverse) drift. Negative ions would be extracted from the other end across slit or cylindrical apertures with a uniform plasma density in the extraction area. Drift waves rotating in the transverse direction will possibly occur in the magnetized region increasing electron transport across the filter (but still the axial electron flux reduction would be substantial). Note that the coextracted electron current can in principle be rendered negligible with an infinite slit (or similarly with the shape of a race-track) surrounded by a cusp magnetic field across the aperture length [145].

\subsection{NBI complexity and its control}

Reliable operation of NBI remains an open issue, as it is the result of several processes, mutually interacting often in a non-linear way. Given these characteristic, the NBI system can be regarded as an example of a complex network, whose controllability can be investigated and tackled by novel tools offered by the theory of complex systems and their controllability [146].

\subsubsection{Complex Network Theory}

Complex network theory is based on the assumption that systems can be described by a Linear Time-Invariant (LTI) approximation, that is a system where the vector $\mathrm{x}(\mathrm{t})$ represents the state of the system variables and $u(t)$ the state of external inputs. A and $\mathrm{B}$ are constant matrices containing the coefficients that link the system together:

$$
\dot{x}=A x+B u .
$$

The system can also be seen from a graphtheoretical point of view: each process or phenomenon inside the system is associated to a state variable and becomes a node of the system. If node $i$ has influence on node $j$ within the system, then there is a link pointing from $i$ to $j$. By definition a system is controllable when it can be driven from any initial state in any final state in finite time [147]. It has been proven [148] that by controlling a subset of the system nodes, namely the driver nodes, the whole system can be driven towards any state.

\subsubsection{Application to NIO1}

NIO1 (Sect. 3.2) constitutes an ideal case as at present it operates without caesium and neutralizer so that the network of processes is limited in number and in links: a total of 40 processes (the nodes of the network) were identified. These processes are listed in Table 1 where a number has been associated to each process in order to easily identify them.

Tab. 1. NIO1 graph nodes where ER is the Extraction Region and PDF is the Particle Distribution Function.

\begin{tabular}{|c|c|}
\hline $\mathbf{N}$ & Description \\
\hline 1 & Divergence of the beam \\
\hline 2 & Beam aiming error \\
\hline 3 & Beam particle energy \\
\hline 4 & $e^{-}$extracted current/ion ext. current \\
\hline 5 & Beam shape asymmetry \\
\hline 6 & Temperature of mechanical \\
\hline 7 & Accelerator breakdown probability \\
\hline 8 & Ion extracted current $\left(I_{H^{-}}\right)$ \\
\hline 9 & Fraction of dissociation $n_{H} / n_{H 2}$ \\
\hline 10 & Source pressure $\left(p_{\text {source }}\right)$ \\
\hline 11 & Gas temperature in driver region \\
\hline 12 & Deviation from maxwellian $e^{-}$PDF \\
\hline 13 & Hot $e^{-}$temperature in extraction \\
\hline 14 & Cold $e^{-}$temperature in ER \\
\hline 15 & Energy of $H_{0}$ impinging on plasma \\
\hline 16 & Density of $H^{-}$in ER $\left(n_{H^{-}, \mathrm{ER}}\right)$ \\
\hline 17 & PG meniscus shape angle/Focal \\
\hline 18 & Plasma drift speed in plasma source \\
\hline 19 & Beam shape asymmetry at meniscus \\
\hline 20 & $I_{H^{-}}$collected on extraction grid (EG) \\
\hline 21 & $I_{H^{-}}$collected on acceleration grid \\
\hline 22 & Density of $H_{2}$ in accelerator $\left(n_{H^{2}, \mathrm{ACC}}\right)$ \\
\hline 23 & Density of $H_{2}$ in source $\left(n_{H^{2}, \text { source }}\right)$ \\
\hline 24 & EG meniscus shape angle/Focal \\
\hline 25 & AG meniscus shape angle/Focal \\
\hline 26 & Density of $\mathrm{H}_{2}$ between PG-EG \\
\hline 27 & Temperature of $\mathrm{H}_{2}$ between PG-EG \\
\hline 28 & Gas temperature in ER \\
\hline 29 & Density of $H_{0}$ between PG-EG \\
\hline 30 & Secondary $e^{-}$emission rate at \\
\hline 31 & Beam shape asymmetry at EG exit \\
\hline 32 & Secondary $e^{-}$density in PG-EG \\
\hline 33 & Secondary $e^{-}$density in accelerator \\
\hline 34 & Leaking secondary $e^{-}$current \\
\hline 35 & Back-streaming positive ion current \\
\hline 36 & $\mathrm{H}^{-}$deflection angle between PG-EG \\
\hline 37 & $H^{-}$deflection angle in accelerator \\
\hline 38 & $H^{-}$deflection angle after $\mathrm{AG}$ \\
\hline 39 & Space charge compensation after AG \\
\hline 40 & $H_{2}$ density in vacuum vessel $\left(n_{H 2}\right)$ \\
\hline
\end{tabular}

For NIO1 four driver nodes have been identified [152]. 
1) Source pressure (10). It influences the efficiency of the magnetic filter, as the collisions of electrons with the gas determine the colder bulk and the hotter tail of the electron energy distribution, thus determining the efficiency of the magnetic filter. In this case, the control of the node is relatively simple via the gas injection system.

2) Density of atomic $\mathrm{H}_{0}$ in the gap between $P G$ and EG (29). It is related to the molecule dissociation degree which in turn affects the mass flow outside the ion source and inside the accelerator. Indeed as the dissociated atoms move faster than the molecules, the pressure inside the source tends to decrease with increasing dissociation degree with a beneficial effect on the stripping losses. In principle, it is possible to control this parameter by fine tuning gas injection and pumping system.

3) Density of $\mathrm{H}_{2}$ in the vessel (40). It was found that the plasma density increases with the gas pressure so that the higher is the pressure, the more efficient is the space charge compensation effect on the beam. However, the density of molecular hydrogen in the vessel affects also the stripping losses in the accelerator region so that the optimal condition is a balance between beneficial effect on the beam optics and the detrimental stripping losses. As a result this driver node can be controlled by an effective differential pumping in the different regions of the NBI system.

4) Deflection of $\mathrm{H}^{-}$in the PG-EG gap (36). It is related to some asymmetry in the meniscus due to plasma drifts induced by the magnetic filter configuration (see Sect. 4.1). This result indicates that a suitable fine tuning of the magnets strength and configuration, as done in [149] or even better an active control the magnetic fields combined with a similar control of the plasma parameters could contribute to a better control of the meniscus formation and then a mitigation of the beam deflection.

To test the predictions of network control principles on NIO1 network the following method was adopted: an input signal was injected into the system and its effects on the output nodes were measured. An output node is a node of the system whose state describes one of the relevant physical outputs of the systems. Six major inputs were selected for the comparison: source pressure $p_{\text {source }}$ (with cryopump turned on and off ), RF power injected in the plasma source $\mathrm{P}_{\mathrm{RF}}$, plasma grid filter current $\mathrm{I}_{\mathrm{PGF}}$, extraction voltage $\mathrm{V}_{\mathrm{EXT}}$ and acceleration voltage $\mathrm{V}_{\mathrm{ACC}}$. For each parameter, scans were selected and the following outputs were considered: extracted current $\mathrm{I}_{\mathrm{EXT}}$, divergence $\theta$, beam aiming error $\delta$, beam particle energy $\epsilon$ and the ratio between extracted electron and ion currents $\mathrm{j}_{\mathrm{e}} / \mathrm{j}_{\mathrm{H}}$. For each scan, a simulation was run with NIO1 graph model to mimic the experimental data and look for correlations. The overall result looks promising: a good correspondence between model and data exists in pressure scans (both with and without cryopump), filter current scans, extraction voltage scans and RF power scans (see Fig. 30), where trends of beam energy, $I_{\text {beam }}$ and

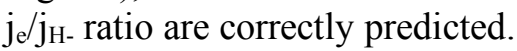
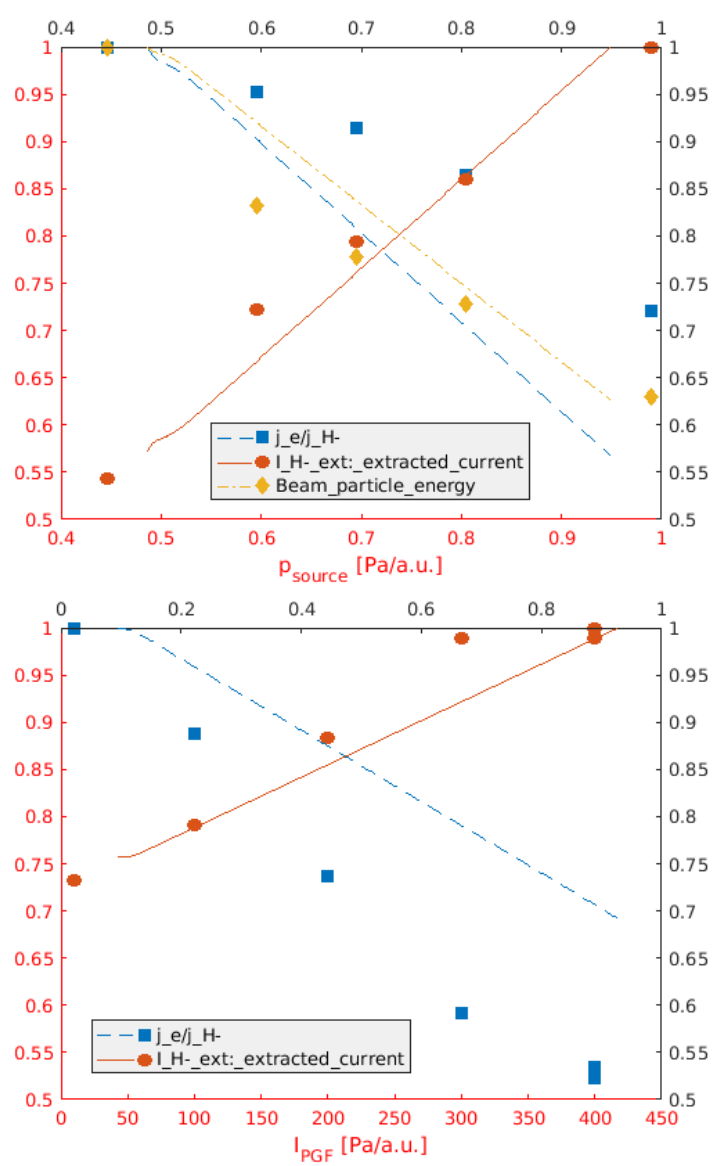

Fig. 30 NIO1 experimental data (dots, left y-axis) compared with NIO1 LTI system solutions (lines, right y-axis). Values from both experimental data and the model are normalized to their maximum value; units of measurement of the bottom $\mathrm{x}$-axis are indicated, units of measurement of top $\mathrm{x}$-axis are arbitrary. (a) Scan in source pressure, $\mathrm{V}_{\mathrm{ACC}}=2.5 \mathrm{kV}, \mathrm{V}_{\mathrm{EXT}}=350 \mathrm{~V}, \mathrm{P}_{\mathrm{RF}}=1.2$ $\mathrm{kW}, \mathrm{I}_{\mathrm{PGF}}=10 \mathrm{~A}$. (b) Scan in IPGF (plasma grid filter current), $\mathrm{V}_{\mathrm{ACC}}=4 \mathrm{kV}, \mathrm{V}_{\mathrm{EXT}}=450 \mathrm{~V}, \mathrm{P}_{\mathrm{RF}}=1.4 \mathrm{~kW}$, $\mathrm{P}_{\text {source }}=0.75 \mathrm{~Pa}$ (beam energy data not available).

\subsubsection{Extension to the whole NBI system: role of surfaces}

In order to extend the model to the whole NBI system, other key processes have to be included involving surface generation and high voltage acceleration. For the surface generation, results confirm that grid and surface temperatures must be separate knobs of the system, that the impurity content must be kept below some critical value and 
that plasma potential, i.e. plasma parameters in the source must be monitored, being aware that the behavior for hydrogen and deuterium could be different. The high voltage holding is of fundamental importance when operating the accelerator at a voltage close to $1 \mathrm{MV}$ as that required for a fusion reactor.

The acceleration voltage has to be achieved with an acceptable breakdowns rate, to allow long duration pulse or steady operation of the NBI. A key role in the breakdown occurrence is played by small electron current emitted by the cathode surface which is generated, even by ideal surfaces, above a certain electric field value due to tunneling process $[150,151]$. The emission of these electrons leads to an increase of the electric field inside the dielectric layer that, in certain condition, exceeds the dielectric strength of layer itself giving rise to current bursts that can evolve into disruptive breakdown if the voltage is sufficiently high (Total Voltage Effect). In terms of controllability accurate and timely measurements of burst amplitude and frequency can in principle anticipate the occurrence of breakdowns during conditioning and potentially during the operation.

\section{Conclusion}

In order to meet the requirements for the ITER tokamak, a first-of-its-kind scientific effort has been undertaken in Europe. Such a work allowed the commissioning of high power $(5-75 \mathrm{~kW})$, high density $\left(\sim 10^{16} \mathrm{~m}^{-3}\right) \mathrm{H}^{-}$negative ion plasma in several institutions (IPP-Garching, RFXCondorzio, SPC-Lausanne). However, several global aspects such as the reliability and the efficiency of the NBI are still not validated for ITER which is not an industrial reactor as DEMO would be, with higher level of requirements.

To meet these goals, these contributions reveal the necessity to address not only the fundamental aspects of the NIS plasma to improve the global efficiency of the NBI. If modern experimental and numerical methods offer the possibility to explore plasma physics, a significant modification of the neutral beam injectors should be considered to take advantages of this common scientific work pursued in Europe.

To summarize the different efforts towards the alternative to Cs solutions reported in this review, one can list the following achievements:

- A single ECR (2.45 GHz) dipolar plasma source has proven its ability to mimic, at lower power input, plasma conditions observed in the RAID reactor. Thanks to its small size ECR plasma has been scrutinized on the DESIRS beamline at
SOLEIL synchrotron with Fourier transform absorption spectroscopy in the VUV range (VUVFTS). VUV-FTS allows absolute measurements of the vibrational distribution functions of molecules and of the atom density for different plasma-facing materials (Quartz, tantalum, tungsten and stainless steel). Tantalum improves significantly the production of vibrationally excited molecules which are essential to the production of negative ions.

- A low work-function (WF) electride material $\left(12 \mathrm{CaO} \cdot 7 \mathrm{Al}_{2} \mathrm{O}_{3}\left(4 \mathrm{e}^{-}\right)\right)$has been tested as caesium alternative. It has demonstrated its ability to enhance negative-ion surface production in hydrogen plasma, despite its WF was not yet optimized. Therefore, this robust, stable at air and machinable conductive ceramic is considered as a promising alternative to caesium.

- In Sect. 3.1 a combination of independent elementary ECR $(2.45 \mathrm{GHz})$ sources has been used for the production of self-sustained hydrogen and deuterium plasmas in a wide range of pressure, demonstrating a scalable source. Species promoting the negative ion volume production are highly yielded. These include high energy electrons for the production of ro-vibrationally excited molecules in the ECR zones and low energy electrons for the production of negative ions by means of dissociative attachment. Thus, densities of $\mathrm{H}^{-}$and $\mathrm{D}^{-}$ions up to $6 \times 10^{9} \mathrm{~cm}^{-3}$ are achieved with a total microwave power less than 1 $\mathrm{kW}$.

- In Sec. 3.2, NIO1 experience of $\mathrm{H}^{-}$production after gas conditioning effect was summarized, discussing its relation with the complex physics of colder plasmas (evidenced by reduced luminosity), filter field and of RF inductive coupling to plasmas (evidenced by resonance frequency shifts).

- In Sec. 3.3, the RAID $10 \mathrm{~kW}$ helicon plasma at the Swiss Plasma Center (EPFL) is sustained by a birdcage resonant antenna, with improved stability and parameter space compared to conventional partial-helix antennas. Significant volume fraction of $\mathrm{H}^{-}$and $\mathrm{D}^{-}$are produced, and preliminary extraction experiments are underway.

- An analysis of the complex network of processes occurring in a negative ion source without Cesium has allowed four main processes to be identified as the key ones to control the whole system

\section{Acknowledgment}

The work of Section 2.2 was carried out within the framework of the French Federation for Magnetic Fusion Studies (FR-FCM) and received some funding 
from FR-FCM.

Concerning Section 2.3, the C12A7-Electride was supplied from AGC Inc. The authors would like to thank H. Hosono for the use of C12A7-Electride, and technical support by Naomichi Miyakawa, Satoru Watanabe, and Kazuho of AGC Inc. It has also received funding from the Excellence Initiative of Aix-Marseille UniversityA*Midex, a French "Investissements d'Avenir programme" AMX-19-IET-013.

The work presented in Section 3.1 was partially supported by the "Andreas Mentzelopoulos" $\mathrm{PhD}$ Scholarships of the University of Patras (M. Mitrou).

Work presented in Section 3.2 set up in collaboration and financial support of INFN (project INFN-E and Group 5, experiments Plasma4beam and Ion2neutral). Work presented in Section 3.2 gratefully acknowledges the financial support of the Swiss National Science Foundation and the mechanical and electronic workshops of the Swiss Plasma Center for their collaboration.

For the work presented in Section 4.1, authors gratefully acknowledge financial support of the Commissariat à l"Energie Atomique (CEA) and the French Fédération de Recherche sur la Fusion Magnétique (FR-FCM).

In addition, works reported in Sections 2.2, 2.3, 3.2, 3.3 and 4.1.3 have also been carried out within the framework of the EUROfusion Consortium and has received funding from the Euratom research and training program 2014-2018 and 2019-2020 under grant agreement No. 633053. The views and opinions expressed herein do not necessarily reflect those of the European Commission.

\section{References}

1. L. W. Alvarez, Rev. Sci. Instrum. 22, 705 (1951)

2. D. Wünderlich et al., Rev. Sci. Instrum. 90, 113304 (2019)

3. J. Lettry, et al. Rev. Sci. Instrum. 87 02B139 (2016)

4. R. F. Welton et al., Rev. Sci. Instrum. 87, 02B146 (2016)

5. M. Yoneda et al., Proceedings of the Ninth International Conference on Accelerator Mass Spectrometry (2004), pp. 223-224

6. V. Toigo et al, New J. Phys. 19, 085004 (2017)

7. P. Agostinetti et al, Fusion Eng. Des. 159, 111628 (2020)

8. A. Simonin et al. Nucl. Fusion 61, 046003 (2021)

9. M. Capitelli, M. Dilonardo, E. Molinari, Chem. Phys. 20, 417 (1977)

10. M. Cacciatore, M. Capitelli, M. Dilonardo, Chem. Phys. 34, 193 (1978)

11. M. Bacal, G.W. Hamilton, Phys. Rev. Lett. 42, 1538 (1979)

12. J. R. Hiskes, J. Appl. Phys. 51, 4592 (1980)

13. J. R. Hiskes, Appl. Phys. Lett. 69, 755 (1996)

14. J. R. Hiskes, A. M. Karo, J. Appl. Phys. 56, 1927 (1984)

15. J. R. Hiskes, A. M. Karo, Appl. Phys. Lett. 54, 508
(1989)

16. J. N. Bardsley, J. M. Wadehra, Phys. Rev. A20, 1398 (1979)

17. J. M. Wadehra, in Nonequilibrium vibrational kinetics - Topics in Current Physics vol 39, Edited by M. Capitelli (Springer-Verlag Berlin, 1986), pp. 191-232

18. R. Celiberto, R. K. Janev, A. Laricchiuta, M. Capitelli, J. M. Wadehra, D. E. Atems, Atom. Data Nucl. Data Tables 77, 161 (2001)

19. J. Bretagne, G. Delouya, C. Gorse, M. Capitelli, M. Bacal, J. Phys. D: Appl. Phys. 18, 811 (1985)

20. C. Gorse, R. Celiberto, M. Cacciatore, A. Laganà, M. Capitelli, Chem. Phys. 161, 211 (1992)

21. C. Gorse, M. Capitelli, J. Bretagne, M. Bacal, Chem. Phys. 93, 1 (1985)

22. C. Gorse, M. Capitelli, M. Bacal, J. Bretagne, A. Laganà, Chem. Phys. 117, 177 (1987)

23. M. Capitelli et al., Nucl. Fus. 46, S260 (2006)

24. A. Laricchiuta, R. Celiberto, F. Esposito, M. Capitelli, Plasma Sources Sci. Technol. 15, S62 (2006)

25. R. Celiberto, M. Capitelli, U. Lamanna, Chem. Phys. 183, 101 (1994)

26. L. H. Scarlett, J. K. Tapley, J. S. Savage, D. V. Fursa, M. C. Zammit, I. Bray, Plasma Sources Sci. Technol. 28, 025004 (2019)

27. J. Hiskes, J. Appl. Phys. 70, 3409 (1991)

28. J. K. Tapley, L. H. Scarlett, J. S. Savage, M. C. Zammit, D. V. Fursa, I. Bray, J. Phys. B: Atom., Mol. Opt. Phys. 51, 144007 (2018)

29. J. K. Tapley, L. H. Scarlett, J. S. Savage, D.V. Fursa, M. C. Zammit, I. Bray, Phys. Rev. A 98, 032701 (2018)

30. G. Colonna, L. D. Pietanza, G. D’Ammando, R. Celiberto, M. Capitelli, A. Laricchiuta, Europ. Phys. J. D 71, 1 (2017)

31. C. S. Trevisan, J. Tennyson, Plasma Phys. Control. Fus. 44, 1263 (2002)

32. D. T. Stibbe, J. Tennyson, New J. Phys. 1, 2 (1998)

33. T. N. Rescigno, B. I. Schneider, J. Phys. B: Atom., Mol. Opt. Phys. 21, L691 (1988)

34. M. A. Khakoo, J. Segura, J. Phys. B: Atom., Mol. Opt. Phys. 27, 2355 (1994)

35. M. A. Khakoo, S. Trajmar, R. McAdams, T. W. Shyn, Phys. Rev. A35, 2832 (1987)

36. H. Nishimura, A. Danjo, J. Phys. Soc. Japan 55, 3031 (1986)

37. R. Celiberto et al., Plasma Sources Sci. Technol. 25, 033004 (2016)

38. M. Zawadzki et al., Phys. Rev. A 98, 062704 (2018)

39. R. Celiberto, R. K. Janev, V. Laporta, J. Tennyson, J. M. Wadehra, Phys. Rev. A 88, 062701 (2013)

40. R. Celiberto, R. Janev, J. Wadehra, J. Tennyson, Chemical Physics, Special Issue Chemical Physics of Low-Temperature Plasmas (in honour of Prof Mario Capitelli) 398, 206 (2012)

41. J. Horáček, M. Čížek, K. Houfek, P. Kolorenč, W. Domcke, Phys. Rev. A 73, 022701 (2006)

42. J. Horáček, M. Č́žžek, K. Houfek, P. Kolorenč, W. Domcke, Phys. Rev. A 70, 052712 (2004) 
43. L. A. Pinnaduwage, W. X. Ding, D. L. McCorkle, S. H. Lin, A. M. Mebel, A. Garscadden, J. Appl. Phys. 85, 7064 (1999)

44. K. Hassouni, A. Gicquel, M. Capitelli, Chem. Phys. Lett. 290, 502 (1998)

45. F. Esposito, C. Gorse, M. Capitelli, Chem. Phys. Lett. 303, 636 (1999)

46. F. Esposito, M. Capitelli, J. Phys. Chem. A 113, 15307 (2009)

47. F. Esposito, C. M. Coppola, D. De Fazio, J. Phys. Chem. A 119, 12615 (2015)

48. M. Capitelli, R. Celiberto, G. Colonna, F. Esposito, C. Gorse, K. Hassouni, A. Laricchiuta, S. Longo, Fundamental Aspects of Plasma Chemical Physics: Kinetics, Vol. 85 (Springer Series on Atomic, Optical, and Plasma Physics, 2016)

49. F. Esposito, Rendiconti Lincei. Scienze Fisiche e Naturali 30, 57 (2019)

50. F. Esposito, R. Macdonald, I. D. Boyd, K. Neitzel, D. A. Andrienko, Heavy-Particle Elementary Processes in Hypersonic Flows. In Hypersonic Meteoroid Entry Physics (IOP Publishing, 2019)

51. R. Götting, V. Herrero, J. P. Toennies, M. Vodegel, Chem. Phys. Lett. 137(6), 524 (1987)

52. R. C. Forrey, Phys. Rev. A 88(5), 052709 (2013)

53. R. Hall, I. Čadež, M. Landau, F. Pichou, C. Schermann, Phys. Rev. Lett. 60, 337 (1988)

54. P. J. Eenshuistra, J. H. M. Bonnie, J. Los, H. J. Hopman, Phys. Rev. Lett. 60, 341 (1988)

55. S. Béchu, et al, J. Quant. Spect. Rad. Transf. 257, 107325 (2020)

56. M. Cacciatore, M. Rutigliano, Plasma Sources Sci. Technol. 18, 023002 (2009)

57. M. Cacciatore, M. Rutigliano, Phys. Scr. T124, 80 (2006)

58. M. Rutigliano, M. Cacciatore, G. D. Billing, Chem. Phys. Lett. 340, 13 (2001)

59. M. Cacciatore, M. Capitelli, G. D. Billing, Surf. Sci. 217, L391 (1989)

60. T. Mosbach, Plasma Sources Sci. Technol. 14, 610 (2005)

61. D. Pagano, C. Gorse, M. Capitelli, IEEE Trans. Plasma Sci. 35, 1247 (2007)

62. K. J. Chung, J. J. Dang, J. Y. Kim, W. H. Cho, Y. S. Hwang, New J. Phys. 18, 105006 (2016)

63. A. Hatayama et al., New J. Physics 20, 065001 (2018)

64. S. Aleiferis, P. Svarnas, S. Béchu, O. Tarvainen, M. Bacal, Plasma Sources Sci. Technol. 27, 075015 (2018)

65. J. Bretagne, G. Delouya, M. Capitelli, C. Gorse, M. Bacal, J. Phys. D: Appl. Phys. 19, 1197 (1986)

66. P. Eenshuistra, R. Heeren, A. Kleyn, H. Hopman, Phys. Rev. A 40, 3613 (1989)

67. J. Bretagne, W. G. Graham, M. B. Hopkins, J. Phys. D: Appl. Phys. 24, 668 (1991)

68. C. Gorse, M. Bacal, R. Celiberto, M. Capitelli, Chem. Phys. Lett. 192, 161 (1992)

69. D. A. Skinner, A.M. Bruneteau, P. Berlemont, C. Courtille, R. Leroy, M. Bacal, Phys. Rev. E 48, 2122 (1993)

70. M. Bacal, M. Wada, Plasma Sources Sci. Technol.
29, 033001 (2020)

71. M. Bacal, M. Wada, Appl. Phys. Rev. 2, 021305 (2015)

72. R. Agnello, et al., Nucl. Fus. 60, 026007 (2020)

73. M. Berger, et al., Plasma Sources Sci. Technol. 18, 025004 (2009)

74. S. Béchu, et al., Phys. Plasmas 20, 101601 (2013)

75. C. Marini, et al., Nucl. Fus. 57(3), 36024 (2017)

76. M. Bacal et al. Rev. Sci. Instrum. 751699 (2004)

77. N. de Oliveira, et al., J. Synchrotron Radiat. 23, 887 (2016)

78. O. Gabriel, et al., Phys. Rev. E 78(1), 16407 (2008)

79. J. Bentounes, et al., Plasma Sources Sci. Technol. 27, 055015 (2018)

80. S. Béchu, et al., Appl. Phys. Lett. 111, 074103 (2017)

81. H. Hosono, Japanese J. Appl. Phys. 52, 090001 (2013)

82. Y. Toda et al., Adv. Mat. 19, 3564 (2007)

83. A. Ahmad et al., Plasma Sources Sci. Technol. 22, 025006 (2013)

84. J. P. J. Dubois et al., J. Appl. Phys. 119, 193301 (2016)

85. D. Kogut et al., Plasma Sources Sci. Technol. 26, 045006 (2017)

86. G. Cartry et al., New J. Phys. 19, 025010 (2017)

87. L. Schiesko et al., Appl. Phys. Lett. 95, 191502 (2009)

88. L. Schiesko et al, Plasma Sources Sci. Technol. 19, 045016 (2010)

89. D. Kogut et al, J. Phys. D: Appl. Phys. 52, 435201 (2019)

90. L. Schiesko et al, Plasma Sources Sci. Technol. 17, 035023 (2008)

91. G. Cartry et al, Phys. Plasmas 19, 063503 (2012)

92. A. Aanesland et al, IEEE Trans. Plasma Sci. 43, 321 (2015)

93. D. Rafalskyi, S. Dudin, A. Aanesland, Rev. Sci. Instrum. 86, 053302 (2015)

94. S. A. Fuselier et al., Space Sci. Rev. 146, 117 (2009)

95. R. Friedl, U. Fantz, J. Appl. Phys. 122, 083304 (2017)

96. A. Heiler et al, J. Vac. Sci. Techn. A39, 013002 (2021)

97. A. Heiler et al, accepted on AIP conference series (2021)

98. C. Wimmer, U. Fantz and NNBI-Team, J. Appl. Phys. 120, 073301 (2016)

99. S. Aleiferis, O. Tarvanien, P. Svarnas, M. Bacal, S. Béchu, J. Phys. D: Appl. Phys. 49, 095203 (2016)

100. R. Gobin, P. Auvray, M. Bacal, J. Breton, O. Delferrière, F. Harrault, A. A. Ivanov Jr., P. Svarnas, O. Tuske, Nucl. Fus. 46, S281 (2006)

101. S. Aleiferis, P. Svarnas, Rev. Sci. Instrum. 85(12), 123504 (2014)

102. M. Bacal, Rev. Sci. Instrum. 71, 3981 (2000)

103. W. Yang, S. N. Averkin, A. V. Khrabrov, I. D. Kaganovich, Y-N. Wang, S. Aleiferis, P. Svarnas, Phys. Plasmas 25, 113509 (2018)

104. D. Wagner, B. Dikmen, H. F. Döbele, Plasma Sources Sci. Technol. 7, 462 (1998) 
105. K. A. Miller, H. Bruhns, M. Čížek, J. Eliášek, R. Cabrera-Trujillo, H. Kreckel, A. P. O’Connor, X. Urbain, D. W. Savin, Phys. Rev. A86, 032714 (2012).

106. W. Kraus et al, Rev. Sci. Instrum. 83, 02B104 (2012)

107. J. Peters, Rev. Sci. Instrum. 79, 02 A515 (2008)

108. D. Wunderlich et al, Plasma Sources Sci. Technol. 23, 015008 (2014)

109. G. Serianni et al, New J. Phys. 19, 045003 (2017)

110. M. P. Stockli et al, AIP Conference Proceedings 1390, 123 (2011)

111. G. Chitarin et al, Rev. Sci. Instrum. 85, 02B317 (2014)

112. M. Cavenago, P. Veltri, Plasma Sources Sci. Technol. 23, 065024 (2014)

113. A. Hatayama, Rev. Sci. Instrum. 79, $02 B 901$ (2008)

114. S. H. Song et al, Phys. Plasmas 21, 093512 (2014)

115. F. F. Chen, IEEE Trans Plasma Sci 34, 718 (2006)

116. M. Cavenago, AIP Conference Proceedings 2052, 020001 (2018)

117. M. Cavenago et al, AIP Conference Proceedings 2052, 040013 (2018)

118. M. Cavenago et al, Rev. Sci. Instrum. 91, 013316 (2020)

119. M. Barbisan et al, Rev. Sci. Instrum, 85, 02A708 (2014)

120. M. Ugoletti et al, to appear in Rev Sci Instrum.

121. J. Palak et al, IEEE Access 6, 29665 (2018)

122. V. Variale et al, Rev. Sci. Instrum. 91, 013516 (2020)

123. M. Cavenago et al., Progress on NIO1 ion source and on energy recover tests, to be presented at IAEA-FEC (2020-2021)

124. M. Cavenago, S. Petrenko, Rev. Sci. Instrum. 83, 02B503 (2012) and references therein

125. I. Furno et al., EPJ Web of Conferences 157, 03014 (2017)

126. Ph. Guittienne et al, submitted to Plasma Sources, Sci. Technol.

127. R. Agnello et al, Rev. Sci. Instrum. 89, 103504 (2018)

128. R. Agnello et al, Nucl. Fus. 60, 026007 (2020)

129. R. Jacquier et al, Fus. Eng. Des. 146, 1140 (2019)

130. S. Lishev et al, Plasma Sources Sci. Technol. 27, 125008 (2018)

131. G. Hagelaar et al, Plasma Sources Sci. Technol. 20, 015001 (2011)

132. J. P. Boeuf et al, Plasma Sources Sci. Technol. 20, 015002 (2011)

133. G. Fubiani et al., New J. Phys. 19, 015002 (2017)

134. F. Taccogna et al., Plasma Sources Sci. Technol. 20(2), 024009 (2011)

135. F. Taccogna, P. Minelli, New J. Phys. 19, 015012 (2017)

136. G. Fubiani et al., Plasma Sources Sci. Technol. 24, 055001 (2015)

137. R. K. Janev, Elementary Processes in HydrogenHelium Plasmas (Springer, 1987)

138. R. McAdam et al., Plasma Sources Sci. Technol. 20, 035023 (2011)
139. G. Fubiani et al., Phys. Plasmas 20, 113511 (2013)

140. P. McNeely et al., Plasma Sources Sci. Technol. 20, 045005 (2011)

141. Seidl et al., J. Appl. Phys. 79, 2896 (1996)

142. S. Geng et al., Fusion Engineering and Design 123, 481 (2017)

143. M. Kashiwagi et al., Rev. Sci. Instrum. 85, 02B320 (2014)

144. G. Fubiani et al., Phys. Plasmas 25, 023510 (2018)

145. Fubiani et al., Phys. Plasmas 27, 093506 (2020)

146. Y. Y. Liu, J. J. Slotine, A. L. Barabasi, Nature 473, $167(2011)$

147. R. E. Kálmán, J. SIAM 1, 152 (1963)

148. C. Lin, IEEE Trans. Automatic Control 19, 3 (1974)

149. G. Chitarin et al, in press on Fusion Engineering and Design

150. R. H. Fowler, L. Nordheim, Proc. Royal Soc. London A119, 781 (1928)

151. E. Spada et al., to appear on IEEE Trans. Plasma Sci.

152. V. Antoni, et al., Rendiconti Lincei. Scienze Fisiche e Naturali 30 (2019) 277-285(2019).

153. V. Dudnikov, Development and Applications of Negative Ion Sources, Springer Series on Atomic, Optical, and Plasma Physics, Vol. 110 (2019). 